\title{
Localization of Fučík curves for the second order discrete Dirichlet operator
}

\author{
Petr Nečesal*, Iveta Sobotková \\ Department of Mathematics and NTIS, University of West Bohemia, Univerzitni 8, \\ 30100 Plzeñ, Czech Republic
}

\section{A R T I C L E I N F O}

Article history:

Received 25 January 2021

Available online 16 June 2021

\section{$M S C$ :}

39A 12

39A 70

34B15

\section{Keywords:}

Asymmetric nonlinearities

Fučík spectrum

Difference operators

Chebyshev polynomials of the second kind

Möbius transformation

\section{A B S T R A C T}

In this paper, we deal with the second order difference equation with asymmetric nonlinearities on the integer lattice and we investigate the distribution of zeros for continuous extensions of positive semi-waves. The distance between two consecutive zeros of two different positive semi-waves depends not only on the parameters of the problem but also on the position of one of these zeros with respect to the integer lattice. We provide an explicit formula for this distance, which allows us to obtain a new simple implicit description of all non-trivial Fučík curves for the discrete Dirichlet operator. Moreover, for fixed parameters of the problem, we show that this distance is bounded and attains its global extrema that are explicitly described in terms of Chebyshev polynomials of the second kind. Finally, for each non-trivial Fučík curve, we provide suitable bounds by two curves with a simple description similar to the description of the first non-trivial Fučík curve.

() 2021 Elsevier Masson SAS. All rights reserved.

\footnotetext{
* Corresponding author.

E-mail addresses: pnecesal@kma.zcu.cz (P. Nečesal), looseova@kma.zcu.cz (I. Sobotková).
} 


\section{Introduction}

In 1976, two papers [10] by Fučík and [5] by Dancer were published concerning the solvability of the following Dirichlet problem

$$
\left\{\begin{array}{l}
v^{\prime \prime}(x)+g(v(x))=f(x), \quad x \in(0,1), \\
v(0)=v(1)=0
\end{array}\right.
$$

where $g$ is a jumping nonlinearity, i.e., $\lim _{s \rightarrow-\infty} \frac{g(s)}{s}=: a \neq b:=\lim _{s \rightarrow+\infty} \frac{g(s)}{s}$. Both authors independently recognized that the solvability of the problem (1) depends strongly on the fact if there exists a non-trivial solution $v$ of the following problem

$$
\left\{\begin{array}{l}
v^{\prime \prime}(x)+a v^{+}(x)-b v^{-}(x)=0, \quad x \in(0,1), \\
v(0)=v(1)=0,
\end{array}\right.
$$

where $v^{+}$and $v^{-}$are the positive and negative parts of $v$, respectively, i.e. $v^{ \pm}(x):=$ $\max \{ \pm v(x), 0\}$. The following set

$$
\Sigma^{\mathbf{c}}:=\left\{(a, b) \in \mathbb{R}^{2}: \text { the problem (2) has a non-trivial solution } v\right\}
$$

is usually called as the Fučík spectrum for (2) and can be expressed analytically in the following way (see $[10,11])$. The Fučík spectrum $\Sigma^{\mathbf{c}}$ consists of two lines $\mathbf{C}_{0}^{ \pm}:\left(a-\pi^{2}\right)$. $\left(b-\pi^{2}\right)=0$ and countably many curves $\mathbf{C}_{l}^{ \pm}$(see Fig. 1 , left) given by $(j \in \mathbb{N})$

$$
\mathbf{C}_{2 j-1}^{ \pm}: \frac{j \pi}{\sqrt{a}}+\frac{j \pi}{\sqrt{b}}=1, \quad \mathbf{C}_{2 j}^{+}: \frac{(j+1) \pi}{\sqrt{a}}+\frac{j \pi}{\sqrt{b}}=1, \quad \mathbf{C}_{2 j}^{-}: \frac{j \pi}{\sqrt{a}}+\frac{(j+1) \pi}{\sqrt{b}}=1 .
$$

Let us note that for a pair $(a, b) \in \mathbf{C}_{l}^{ \pm}$, the corresponding non-trivial solution $v$ of (2) has exactly $l$ zeros in $(0,1)$ and consists of positive and negative semi-waves of lengths $\frac{\pi}{\sqrt{a}}$ and $\frac{\pi}{\sqrt{b}}$, respectively (see Fig. 1, right).

In 1987, Lazer and McKenna introduced a new nonlinear model of a suspension bridge using the asymmetric nonlinearity $g(v)=k v^{+}$to describe supporting cable stays as onesided springs which do not exert restoring force if they are compressed. They studied periodic solutions of such asymmetric systems and showed in [17] that a sufficiently large asymmetry in the system leads to large oscillations which cannot be predicted by the linear theory. In [8], authors consider the following normalized symmetric model of the vertical motion of a suspension bridge

$$
\left\{\begin{array}{l}
v_{t t}(x, t)+v_{x x x x}(x, t)+k v^{+}(x, t)=f(x, t) \quad \text { in }\left(-\frac{\pi}{2}, \frac{\pi}{2}\right) \times \mathbb{R}, \\
v\left( \pm \frac{\pi}{2}, t\right)=v_{x x}\left( \pm \frac{\pi}{2}, t\right)=0, \quad t \in \mathbb{R}, \\
v(x, t)=v(-x, t)=v(x,-t)=v(x, t+T),
\end{array}\right.
$$



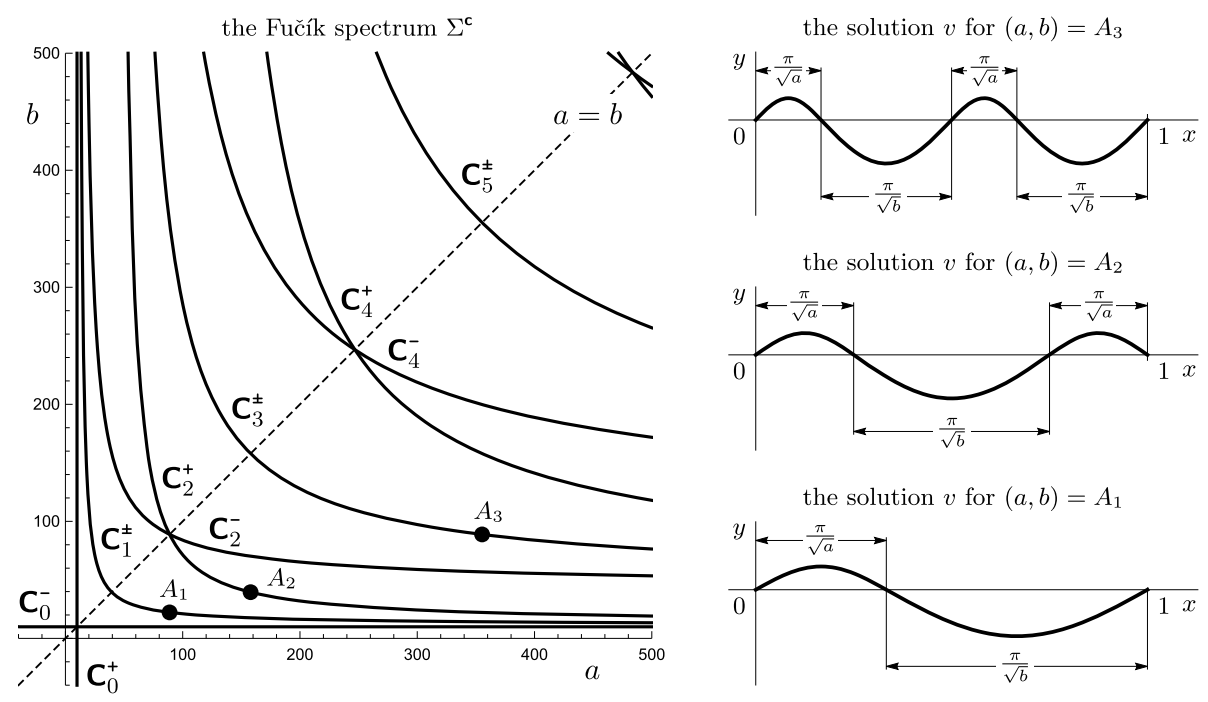

Fig. 1. The Fučík spectrum $\Sigma^{\mathbf{c}}$ (left) for the continuous problem (2) given by countable many Fučík curves $\mathbf{C}_{l}^{ \pm}, l \in \mathbb{N} \cup\{0\}$, and corresponding non-trivial solutions $v$ (right) for three different pairs $(a, b)$ as points $A_{1} \in \mathbf{C}_{1}^{+}, A_{2} \in \mathbf{C}_{2}^{+}$and $A_{3} \in \mathbf{C}_{3}^{+}$, where $A_{1}=\left(9 \pi^{2}, \frac{9}{4} \pi^{2}\right), A_{2}=\left(16 \pi^{2}, 4 \pi^{2}\right)$ and $A_{3}=\left(36 \pi^{2}, 9 \pi^{2}\right)$.

and investigate its set of solutions $v$ that are $T$-periodic in the second variable. For special right hand sides $f$ and $T>\pi$, they show that it has very rich set of non-stationary solutions with blow up points in the sense that for bounded values of the parameter $k$ there are non-stationary solutions of (4) with the amplitude approaching infinity. Let us point out that the blow up points are determined by the Fučík spectrum of the beam operator $v \mapsto-\left(v_{t t}+v_{x x x x}\right)$ with the boundary conditions given in (4). However, the knowledge of the Fučík spectrum of this operator seems to be a hard problem. For other one dimensional models of suspension bridges, we recommend the reader the book [12] by Gazzola with a focus on Subchapter 2.8 concerning models with asymmetric nonlinearities. Finally, let us note that asymmetric nonlinearities also surprisingly appear in the study of competing systems of species with large interactions in biology (see $[4,6,22]$ ) and the Fučík spectrum of the Dirichlet Laplacian (the Laplace operator $u \mapsto$ $-\Delta u$ with zero Dirichlet boundary conditions) is needed (see [6] for details).

Nowadays, there are a number of papers in which authors study the structure of the Fučík spectrum for particular linear differential operators, let us mention here only some of them: $[1,2,7,9,14,23,24]$ for the Dirichlet Laplacian on bounded domains, $[3,13,15,16$, $26,27]$ for the ordinary differential operators with various boundary conditions (Dirichlet, Neumann, Robin, Navier, periodic, multipoint, integral type).

In [22] and [25], authors consider a finite dimensional nonlinear matrix-vector equation

$$
\mathbf{A u}=g(\mathbf{u})
$$


where $\mathbf{A}$ is an $n \times n$ matrix and $g: \mathbb{R}^{n} \rightarrow \mathbb{R}^{n}$ is mildly nonlinear, i.e. $g(\mathbf{u})=a \mathbf{u}^{+}-b \mathbf{u}^{-}+$ $h(\mathbf{u})$, where $h: \mathbb{R}^{n} \rightarrow \mathbb{R}^{n}$ is such that $\lim _{\|\mathbf{u}\| \rightarrow+\infty} \frac{h(\mathbf{u})}{\|\mathbf{u}\|}=\mathbf{0}$. Equations of this type (5) can represent numerical approximations of continuous boundary value problems describing nonlinear oscillations in asymmetric systems such as suspension bridges (see [25] and [18]). The Fučík spectrum of the matrix $\mathbf{A}$ is defined as the set of all pairs $(a, b) \in \mathbb{R}^{2}$ such that the problem $\mathbf{A u}=a \mathbf{u}^{+}-b \mathbf{u}^{-}$has a non-trivial solution $\mathbf{u}$ and plays an important role in questions of the solvability of the discrete equation (5). More precisely, in [22], the solvability of (5) is provided in the so-called nonresonance case when the point $(a, b)$ is not in the Fučík spectrum of $\mathbf{A}$ and can be connected by a continuous curve to a point $(\lambda, \lambda)$ on the diagonal $a=b$ such that this curve belongs to the complement of the Fučík spectrum. In [25], authors investigate the Fučík spectrum of the following tridiagonal persymmetric matrix $(\delta \geq 0)$

$$
\mathbf{A}_{\delta}:=\left[\begin{array}{ccccc}
2+\delta & -(1+\delta) & & & \\
-1 & 2+\delta & -(1+\delta) & & \\
& \ddots & \ddots & \ddots & \\
& & -1 & 2+\delta & -(1+\delta) \\
& & & -1 & 2+\delta
\end{array}\right]
$$

which represents a discrete approximation of the differential operator $u \mapsto-\left(u^{\prime \prime}+\delta u^{\prime}\right)$ with zero Dirichlet boundary conditions. Moreover, the solvability of (5) is investigated in both the resonance and nonresonance case, i.e. when the point $(a, b)$ is, or is not in the Fučík spectrum of $\mathbf{A}_{\delta}$. Finally, at the end of the paper [25], authors leave the reader with two interesting problems and one of them is to determine a complete description of the Fučík spectrum of the $n \times n$ matrix $\mathbf{A}_{\delta}$ for $n \geq 3$. In the special case of $\delta=0$, the Fučík spectrum of $\mathbf{A}_{0}$ has been also studied in [20-22,28] and let us note that its known description for $n \geq 3$ is rather more complicated in comparison to the simple description of Fučík curves $\mathbf{C}_{l}^{ \pm}$given in (3) for the continuous problem (2).

In this paper, we continue in studying the Fučík spectrum of the matrix $\mathbf{A}_{0}$ given in (6) for $\delta=0$ and thus, we deal with the following discrete Dirichlet problem

$$
\left\{\begin{array}{l}
\Delta^{2} u(k-1)+\alpha u^{+}(k)-\beta u^{-}(k)=0, \quad k \in \mathbb{T} \\
u(0)=u(n+1)=0
\end{array}\right.
$$

where $\mathbb{T}:=\{1, \ldots, n\}, n \in \mathbb{N}$, and $u: \widehat{\mathbb{T}} \rightarrow \mathbb{R}, \widehat{\mathbb{T}}:=\mathbb{T} \cup\{0, n+1\}$. Moreover, $\alpha, \beta \in \mathbb{R}$, $\Delta^{2}$ denotes the second order forward difference operator, i.e.

$$
\Delta^{2} u(k-1):=u(k-1)-2 u(k)+u(k+1),
$$

$u^{ \pm}: \widehat{\mathbb{T}} \rightarrow \mathbb{R}$ are positive and negative parts of $u$, i.e. $u^{ \pm}(k):=\max \{ \pm u(k), 0\}$. The aim of this paper is to investigate the Fučík spectrum for the problem (7) as the set 

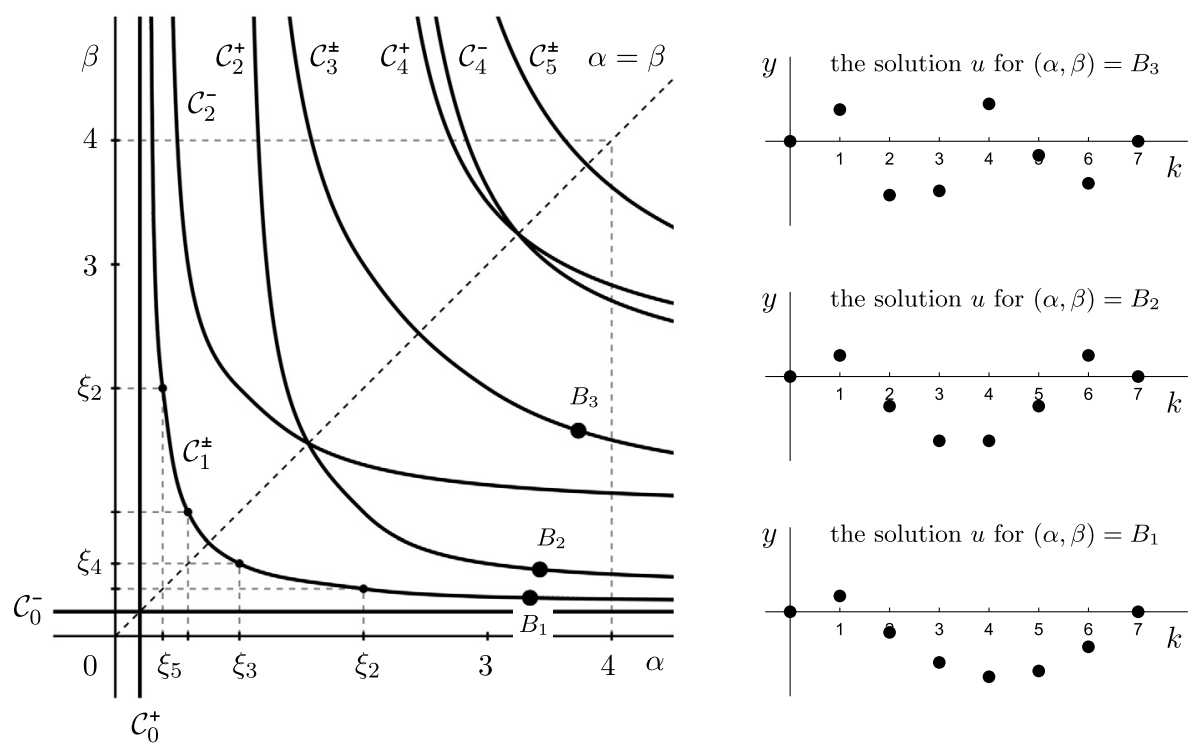

Fig. 2. The Fučík spectrum $\Sigma$ (left) for the discrete problem (7) given by twelve Fučík curves $\mathcal{C}_{0}^{ \pm}, \mathcal{C}_{1}^{ \pm}, \mathcal{C}_{2}^{ \pm}$, $\mathcal{C}_{3}^{ \pm}, \mathcal{C}_{4}^{ \pm}, \mathcal{C}_{5}^{ \pm}$in the case of $n=6$ (note that $\mathcal{C}_{1}^{+}=\mathcal{C}_{1}^{-}, \mathcal{C}_{3}^{+}=\mathcal{C}_{3}^{-}$and $\mathcal{C}_{5}^{+}=\mathcal{C}_{5}^{-}$) and corresponding nontrivial solutions $u$ (right) for three different pairs $(\alpha, \beta)$ as points $B_{1} \in \mathcal{C}_{1}^{+}, B_{2} \in \mathcal{C}_{2}^{+}$and $B_{3} \in \mathcal{C}_{3}^{+}$, where $B_{1} \doteq(3.342,0.309), B_{2} \doteq(3.421,0.538)$ and $B_{3} \doteq(3.732,1.657)$.

$$
\Sigma:=\left\{(\alpha, \beta) \in \mathbb{R}^{2}: \text { the problem }(7) \text { has a non-trivial solution } u\right\}
$$

Let us note that the set $\Sigma$ is exactly the Fučík spectrum of the matrix $\mathbf{A}_{0}$.

Let us briefly recall some known results concerning the set $\Sigma$ (for a more detailed overview see the first section in [20]). The Fučík spectrum consists of a finite number of algebraic curves (see Fig. 2)

$$
\Sigma=\bigcup_{l=0}^{n-1}\left(\mathcal{C}_{l}^{+} \cup \mathcal{C}_{l}^{-}\right)
$$

where

$$
\begin{aligned}
\mathcal{C}_{l}^{ \pm}:=\left\{(\alpha, \beta) \in \mathbb{R}^{2}: \quad\right. & \text { the problem }(7) \text { has a non-trivial solution } u \\
& \text { with exactly } l \text { generalized zeros on } \mathbb{T} \text { and } u(1) \gtrless 0\} .
\end{aligned}
$$

Let us note that $j \in \mathbb{T}$ is a generalized zero of the solution $u$ of (7) if $u(j)=0$ or $u(j) u(j-1)<0$. Fučík curves $\mathcal{C}_{0}^{ \pm}$are trivial ones (lines $\alpha=\lambda_{0}$ and $\beta=\lambda_{0}$, where $\left.\lambda_{0}:=4 \sin ^{2} \frac{\pi}{2(n+1)}\right)$, each non-trivial Fučík curve $\mathcal{C}_{l}^{ \pm}, l \in\{1, \ldots, n-1\}$ is located in the domain $D:=((0,4) \times(0,+\infty)) \cup((0,+\infty) \times(0,4))$. For $\alpha=\beta=\lambda$, the problem (7) is a linear one and thus, it is straightforward to verify that it has a non-trivial solution $u$ if and only if $\lambda=\lambda_{j}:=4 \sin ^{2} \frac{(j+1) \pi}{2(n+1)}, j=0, \ldots, n-1$. Moreover, the corresponding 


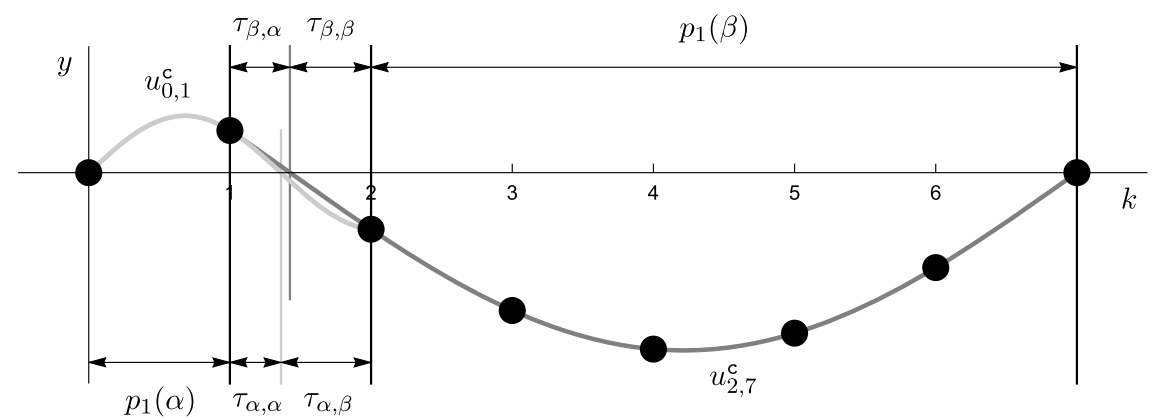

Fig. 3. The geometry of the discrete solution $u$ of $(7)$ for $(\alpha, \beta)=B_{1} \in \mathcal{C}_{1}^{ \pm}$, where $B_{1} \doteq(3.342,0.309)$. The solution $u$ has one generalized zero at $j=2$ and two continuous extensions $u_{0,1}^{\mathrm{c}}$ and $u_{2,7}^{\mathrm{c}}$.

non-trivial solution is $u_{j}(k)=\sin \left(\omega_{\lambda_{j}} k\right) / \sin \omega_{\lambda_{j}}$, where $\omega_{\lambda_{j}}:=\arccos \frac{2-\lambda_{j}}{2}$, and thus, the point $\left(\lambda_{j}, \lambda_{j}\right)$ on the diagonal $\alpha=\beta$ belongs to both Fučík curves $\mathcal{C}_{j}^{+}$and $\mathcal{C}_{j}^{-}$.

The qualitative properties of the first non-trivial Fučík curve $\mathcal{C}_{1}^{ \pm}$were studied in $[20,28]$. In [28], a conjecture is stated that $\mathcal{C}_{1}^{ \pm}$has no elementary parametrization and possible ways to prove it are also discussed. On the other hand, in [20], it is shown that the first non-trivial Fučík curve $\mathcal{C}_{1}^{ \pm}$has an elementary parametrization for $n \leq 7$. The reason is that it is possible to provide the implicit description of $\mathcal{C}_{1}^{ \pm}$in terms of Chebyshev polynomials of the second kind. More precisely, due to Corollary 30 in [20], the first non-trivial Fučík curve $\mathcal{C}_{1}^{ \pm}$consists of the following $(n-1)$ algebraic curves in prescribed rectangles

$$
\begin{array}{rr}
V_{n-1}^{\alpha} \cdot(2-\beta)-V_{n-2}^{\alpha}=0 & \text { for }(\alpha, \beta) \in\left(\xi_{n}, \xi_{n-1}\right) \times\left(\xi_{2},+\infty\right), \\
V_{n-i}^{\alpha} \cdot V_{i}^{\beta}-V_{n-i-1}^{\alpha} \cdot V_{i-1}^{\beta}=0 & \text { for }(\alpha, \beta) \in\left(\xi_{n-i+1}, \xi_{n-i}\right) \times\left(\xi_{i+1}, \xi_{i}\right), \\
(2-\alpha) \cdot V_{n-1}^{\beta}-V_{n-2}^{\beta}=0 & \text { for }(\alpha, \beta) \in\left(\xi_{2},+\infty\right) \times\left(\xi_{n}, \xi_{n-1}\right),
\end{array}
$$

where $V_{k}^{\alpha}$ and $V_{k}^{\beta}$ are defined by the Chebyshev polynomial $U_{k}=U_{k}(x)$ of the second kind of degree $k$

$$
V_{k}^{\lambda}:=U_{k}\left(\frac{2-\lambda}{2}\right), \quad k \in \mathbb{Z}, \lambda \in \mathbb{R},
$$

and the values $\xi_{k}$ for $k=2, \ldots, n$ are given by the formula

$$
\xi_{k}:=4 \sin ^{2} \frac{\pi}{2 k}, \quad k \in \mathbb{N}
$$

Moreover, the first non-trivial Fučík curve $\mathcal{C}_{1}^{ \pm}$contains also $(n-2)$ points $\left(\xi_{n-i}, \xi_{i+1}\right)$, $i=1, \ldots, n-2$ (see Fig. 2 for the Fučík curve $\mathcal{C}_{1}^{ \pm}$in the case of $n=6$, which consists of four points and five algebraic curves). 


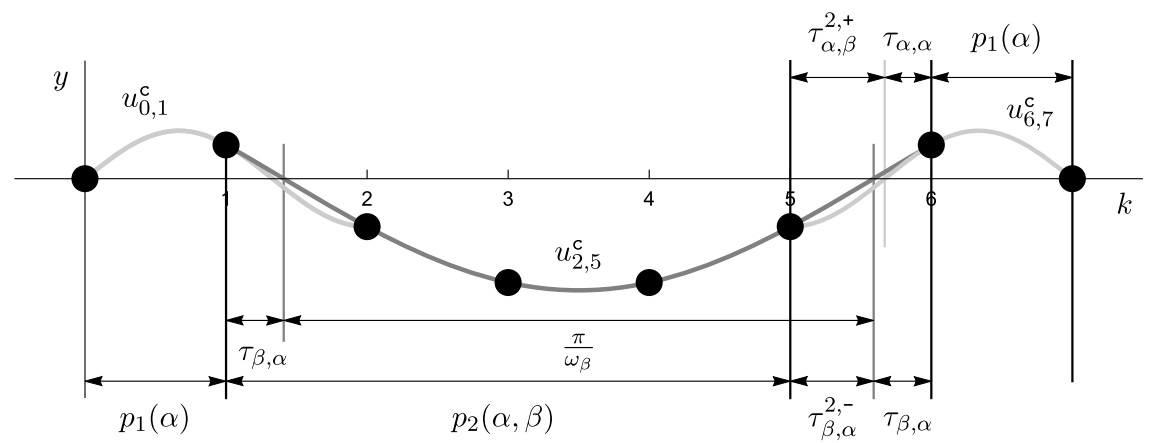

Fig. 4. The geometry of the discrete solution $u$ of $(7)$ for $(\alpha, \beta)=B_{2} \in \mathcal{C}_{2}^{+}$, where $B_{2} \doteq(3.421,0.538)$. The solution $u$ has two generalized zeros $(2$ and 6$)$ and three continuous extensions $u_{0,1}^{\mathrm{c}}, u_{2,5}^{\mathrm{c}}$ and $u_{6,7}^{\mathrm{c}}$.

Now, let us recall the discrete anchoring procedure introduced in [28] which is also called the matching-extension method in [21] and can be used to obtain an implicit description of all Fučík curves $\mathcal{C}_{l}^{ \pm}$. This technique consists of successive anchoring positive and negative continuous semi-waves which are defined as continuous extensions of the discrete solution $u$ of (7) on intervals determined by generalized zeros of $u$. See Figs. 2 and 3 for a non-trivial discrete solution $u$ of $(7)$ for $(\alpha, \beta)=B_{1} \in \mathcal{C}_{1}^{+}$. This discrete solution $u$ has one generalized zero on $\mathbb{T}$ at $j=2$ and thus, we have one positive continuous semi-wave $u_{0,1}^{c}$ on the interval $[0,2]$ and one negative continuous semi-wave $u_{2,7}^{\mathrm{c}}$ on the interval $[1,7]$. These two continuous semi-waves are anchored on the interval $[1,2]$ such that $u_{0,1}^{\mathrm{c}}(1)=u_{2,7}^{\mathrm{c}}(1)$ and $u_{0,1}^{\mathrm{c}}(2)=u_{2,7}^{\mathrm{c}}(2)$. Now, for simplicity, let us consider that $0<\alpha, \beta<4$. By Theorem 26 in [20], the problem (7) has a non-trivial solution $u$ with $u(1)>0$ and exactly one generalized zero on $\mathbb{T}$ if and only if

$$
p_{1}(\alpha)+p_{1}(\beta)+\tau_{\alpha, \alpha}+\tau_{\alpha, \beta}=n+1
$$

where we have denoted

$$
p_{1}(\alpha):=\left\lfloor\frac{\pi}{\omega_{\alpha}}\right\rfloor, \quad p_{1}(\beta)=\left\lfloor\frac{\pi}{\omega_{\beta}}\right\rfloor, \quad \tau_{\alpha, \beta}:=T^{\alpha}\left(\frac{V_{p_{1}(\beta)}^{\beta}}{V_{p_{1}(\beta)-1}^{\beta}}\right), \quad \tau_{\alpha, \alpha}=T^{\alpha}\left(\frac{V_{p_{1}(\alpha)}^{\alpha}}{V_{p_{1}(\alpha)-1}^{\alpha}}\right),
$$

and $\lfloor\cdot\rfloor$ is the floor function, $\omega_{\alpha}:=\arccos \frac{2-\alpha}{2}$ and the function $T^{\alpha}: \mathbb{R}^{*} \rightarrow \mathbb{R}$ with the domain $\mathbb{R}^{*}:=\mathbb{R} \cup\{\infty\}$ (the one-point compactification of $\mathbb{R}$ ) is defined as

$$
T^{\alpha}(\infty):=0, \quad T^{\alpha}(q):=\frac{1}{\omega_{\alpha}} \operatorname{arccot} \frac{\cos \omega_{\alpha}-q}{\sin \omega_{\alpha}} \quad \text { for } q \in \mathbb{R}
$$

Let us point out that the function arccotangent in (12) is strictly decreasing on $\mathbb{R}$ with the range $(0, \pi)$. Thus, a pair $(\alpha, \beta)$ belongs to $\mathcal{C}_{1}^{+}$if and only if (10) holds. Moreover, the equation (10) can be equivalently replaced by 


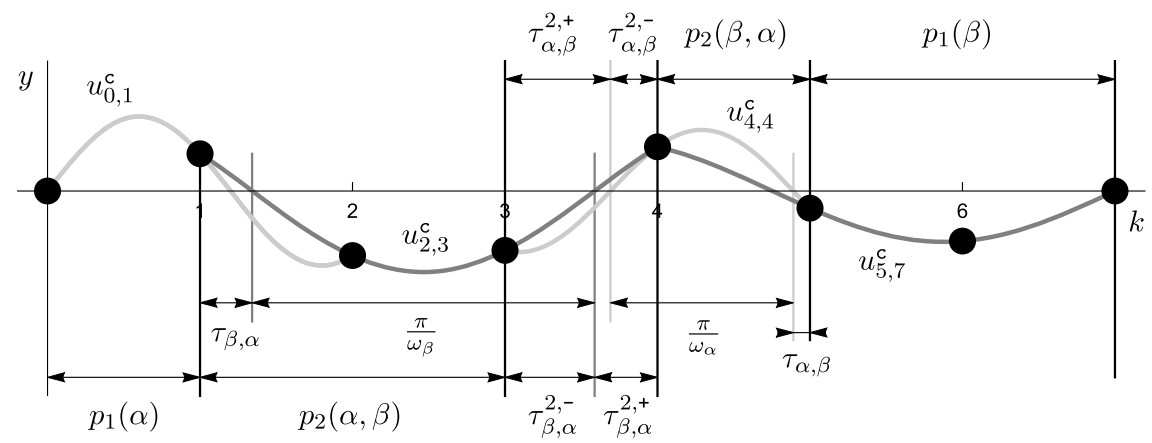

Fig. 5. The geometry of the discrete solution $u$ of $(7)$ for $(\alpha, \beta)=B_{3} \in \mathcal{C}_{3}^{ \pm}$, where $B_{3} \doteq(3.732,1.657)$. The solution has three generalized zeros $(2,4$ and 5$)$ and four continuous extensions $u_{0,1}^{\mathrm{c}}, u_{2,3}^{\mathrm{c}}, u_{4,4}^{\mathrm{c}}$ and $u_{5,7}^{\mathrm{c}}$.

$$
p_{1}(\alpha)+p_{1}(\beta)+\tau_{\beta, \beta}+\tau_{\beta, \alpha}=n+1
$$

Let us point out that if $(\alpha, \beta) \in \mathcal{C}_{1}^{+}$such that $\alpha \neq \beta$ and $\beta \neq \xi_{k}$ for all $k \in\{2, \ldots, n-1\}$ then zeros of the positive and the negative continuous semi-waves do not coincide (see Fig. 3). Indeed, $\left(p_{1}(\alpha)+\tau_{\alpha, \alpha}\right)$ and $\left(p_{1}(\alpha)+\tau_{\beta, \alpha}\right)$ are zeros of the positive and the negative semi-waves, respectively, and we have that $\tau_{\alpha, \alpha}=\tau_{\beta, \alpha}$ if and only if $\alpha=\beta$ or $\beta \in\left\{\xi_{2}, \ldots, \xi_{n-1}\right\}$.

Now, using Theorem 26 in [20], the second non-trivial Fučík curve $\mathcal{C}_{2}^{+}$can be implicitly described as (see Figs. 2 and 4 )

$$
2 p_{1}(\alpha)+p_{2}(\alpha, \beta)+\tau_{\alpha, \alpha}+\tau_{\alpha, \beta}^{2,+}=n+1,
$$

where we have denoted

$$
\begin{aligned}
& p_{2}(\alpha, \beta):=\left\lfloor\tau_{\beta, \alpha}+\frac{\pi}{\omega_{\beta}}\right\rfloor \\
& \tau_{\alpha, \beta}^{2,+}:=T^{\alpha}\left(\frac{V_{p_{1}(\alpha)}^{\alpha} V_{p_{2}(\alpha, \beta)}^{\beta}-V_{p_{1}(\alpha)-1}^{\alpha} V_{p_{2}(\alpha, \beta)-1}^{\beta}}{V_{p_{1}(\alpha)}^{\alpha} V_{p_{2}(\alpha, \beta)-1}^{\beta}-V_{p_{1}(\alpha)-1}^{\alpha} V_{p_{2}(\alpha, \beta)-2}^{\beta}}\right) .
\end{aligned}
$$

As in the previous case, the equation (14) can be equivalently replaced by

$$
2 p_{1}(\alpha)+p_{2}(\alpha, \beta)+\tau_{\alpha, \beta}^{2,-}+\tau_{\beta, \alpha}=n+1,
$$

where we have denoted

$$
\tau_{\alpha, \beta}^{2,-}:=T^{\alpha}\left(\frac{V_{p_{1}(\beta)}^{\beta} V_{p_{2}(\beta, \alpha)}^{\alpha}-V_{p_{1}(\beta)-1}^{\beta} V_{p_{2}(\beta, \alpha)-1}^{\alpha}}{V_{p_{1}(\beta)}^{\beta} V_{p_{2}(\beta, \alpha)-1}^{\alpha}-V_{p_{1}(\beta)-1}^{\beta} V_{p_{2}(\beta, \alpha)-2}^{\alpha}}\right) .
$$

Finally, the third non-trivial Fučík curve $\mathcal{C}_{3}^{+}$can be implicitly described as (see Figs. 2 and 5) 


$$
p_{1}(\alpha)+p_{1}(\beta)+p_{2}(\alpha, \beta)+p_{2}(\beta, \alpha)+\tau_{\alpha, \beta}^{2,+}+\tau_{\alpha, \beta}^{2,-}=n+1
$$

or as

$$
p_{1}(\alpha)+p_{1}(\beta)+p_{2}(\alpha, \beta)+p_{2}(\beta, \alpha)+\tau_{\beta, \alpha}^{2,-}+\tau_{\beta, \alpha}^{2,+}=n+1
$$

Now, if we would like to describe higher Fučík curves then we have to use functions with higher level of nesting depth and also a higher number of different Chebyshev polynomials. Let us only note that to obtain an implicit description of the Fučík curve $\mathcal{C}_{l}^{+}$for $l \geq 4$, we need to use nested functions $p_{3}(\alpha, \beta):=\left\lfloor\tau_{\alpha, \beta}^{2,+}+\frac{\pi}{\omega_{\alpha}}\right\rfloor$ and

$$
\tau_{\alpha, \beta}^{3,+}:=T^{\alpha}\left(\frac{V_{p_{1}}^{\alpha} V_{p_{2}}^{\beta} V_{p_{3}}^{\alpha}-V_{p_{1}-1}^{\alpha} V_{p_{2}-1}^{\beta} V_{p_{3}}^{\alpha}-V_{p_{1}}^{\alpha} V_{p_{2}-1}^{\beta} V_{p_{3}-1}^{\alpha}+V_{p_{1}-1}^{\alpha} V_{p_{2}-2}^{\beta} V_{p_{3}-1}^{\alpha}}{V_{p_{1}}^{\alpha} V_{p_{2}}^{\beta} V_{p_{3}-1}^{\alpha}-V_{p_{1}-1}^{\alpha} V_{p_{2}-1}^{\beta} V_{p_{3}-1}^{\alpha}-V_{p_{1}}^{\alpha} V_{p_{2}-1}^{\beta} V_{p_{3}-2}^{\alpha}+V_{p_{1}-1}^{\alpha} V_{p_{2}-2}^{\beta} V_{p_{3}-2}^{\alpha}}\right),
$$

where $p_{1}=p_{1}(\alpha), p_{2}=p_{2}(\alpha, \beta)$ and $p_{3}=p_{3}(\alpha, \beta)$. If we compare the definitions of $\tau_{\alpha, \beta}, \tau_{\alpha, \beta}^{2,+}$ and $\tau_{\alpha, \beta}^{3,+}$ in (11), (15) and (19), respectively, we have to conclude that the complexity of the known implicit description of Fučík curves $\mathcal{C}_{l}^{ \pm}$substantially increases with increasing numbers of generalized zeros $l$ of the solution $u$. As far as we know, it is not possible to lower the level of used nested functions. Thus, in this paper, for each non-trivial Fučík curve $\mathcal{C}_{l}^{ \pm}$, we provide new bounds with the same description complexity as the implicit description (10) for the first non-trivial Fučík curve $\mathcal{C}_{1}^{ \pm}$. In the following section, we introduce these new bounds and present the main results of this paper.

\section{Main results}

In this section, we introduce two main results of this paper concerning the discrete problem (7), namely Theorems 3 and 5. Proofs of both these theorems are provided in the following sections.

One of the main goals of this paper is to provide new suitable bounds for each non-trivial Fučík curve $\mathcal{C}_{l}^{ \pm}$such that all these bounds will have the same simplicity of description as used in (10) for the first non-trivial Fučík curve $\mathcal{C}_{1}^{ \pm}$. Let us recall that the Fuč́k spectrum $\Sigma$ is symmetric to the diagonal $\alpha=\beta$ and each of its non-trivial Fučík curve $\mathcal{C}_{l}^{ \pm}$is in the domain $D=((0,4) \times(0,+\infty)) \cup((0,+\infty) \times(0,4))$ (see Fig. 6). Thus, it is enough to construct bounds for Fučík curves only in the following half-strip

$$
\mathcal{D}:=(0,4) \times(0,+\infty)
$$

Now, let us define the basic map $\kappa_{\beta}:(0,+\infty) \rightarrow \mathbb{N}_{0}$, where $\mathbb{N}_{0}:=\mathbb{N} \cup\{0\}$, as

$$
\kappa_{\beta}:= \begin{cases}\left\lfloor\frac{\pi}{\omega_{\beta}}\right\rfloor-1 & \text { for } 0<\beta<4 \\ 0 & \text { for } \beta \geq 4\end{cases}
$$




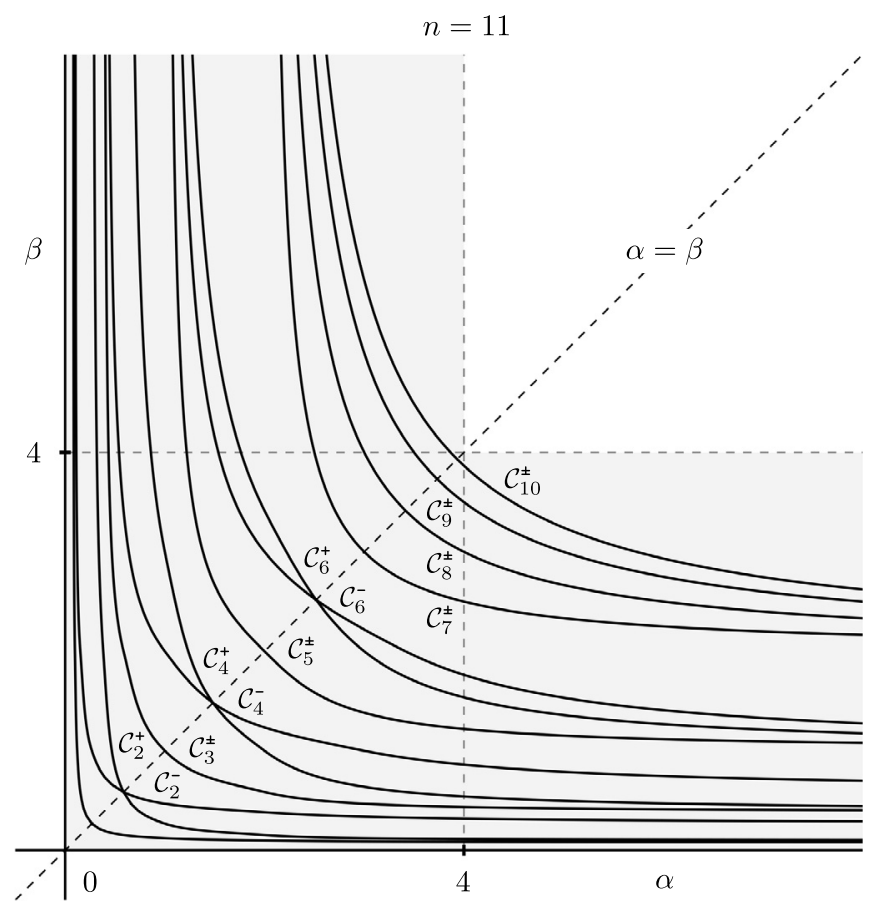

Fig. 6. All non-trivial Fučík curves $\mathcal{C}_{l}^{ \pm}$(black curves) for $l=1, \ldots, n-1$ are contained in the domain $D=((0,4) \times(0,+\infty)) \cup((0,+\infty) \times(0,4))$ (grey region). Let us note that for $n=11$, we have $\mathcal{C}_{l}^{+}=\mathcal{C}_{l}^{-}$for $l=1,3,5,7,8,9,10$.

where $\omega_{\beta}:=\arccos \frac{2-\beta}{2}$ for $0<\beta<4$. Using $\kappa_{\beta}$, we decompose the half-strip $\mathcal{D}$ into rectangles by $\kappa_{\beta}=k, k \in \mathbb{N}_{0}$, i.e. we have (see Fig. 8)

$$
\mathcal{D}=\left((0,4) \times\left(\xi_{2},+\infty\right]\right) \cup\left((0,4) \times\left(\xi_{3}, \xi_{2}\right]\right) \cup \cdots \cup\left((0,4) \times\left(\xi_{k+2}, \xi_{k+1}\right]\right) \cup \ldots,
$$

where $\xi_{k}$ is defined in (9). On each rectangle given by $\kappa_{\beta}=k$, we use Chebyshev polynomials of two degrees $V_{\kappa_{\beta}}^{\beta}$ and $V_{\kappa_{\beta}+1}^{\beta}$ to introduce three basic elements $\eta_{\alpha, \beta}, \tau_{\alpha, \beta}$ and $\mu_{\alpha, \beta}$ in the following definition. Let us note that for fixed $\beta \in(0,4)$, the value $\frac{\pi}{\omega_{\beta}}$ used in (20) represents the distance between zeros of the continuous extension of a negative semi-wave (see Figs. 4 and 5 for continuous extensions $u_{2,5}^{c}$ and $u_{2,3}^{\mathrm{c}}$, respectively).

Definition 1. For $0<\alpha<4$ and $\beta>0$, let us define

$$
\eta_{\alpha, \beta}:=T^{\alpha}\left(\frac{V_{\kappa_{\beta}+1}^{\beta}-1}{V_{\kappa_{\beta}}^{\beta}}\right), \quad \tau_{\alpha, \beta}:=T^{\alpha}\left(\frac{V_{\kappa_{\beta}+1}^{\beta}}{V_{\kappa_{\beta}}^{\beta}}\right), \quad \mu_{\alpha, \beta}:=T^{\alpha}\left(\frac{V_{\kappa_{\beta}+1}^{\beta}}{V_{\kappa_{\beta}}^{\beta}+1}\right)
$$

where the function $T^{\alpha}$ is given by (12) and $V_{k}^{\beta}$ is given in (8) by Chebyshev polynomials of the second type of degree $k$. 


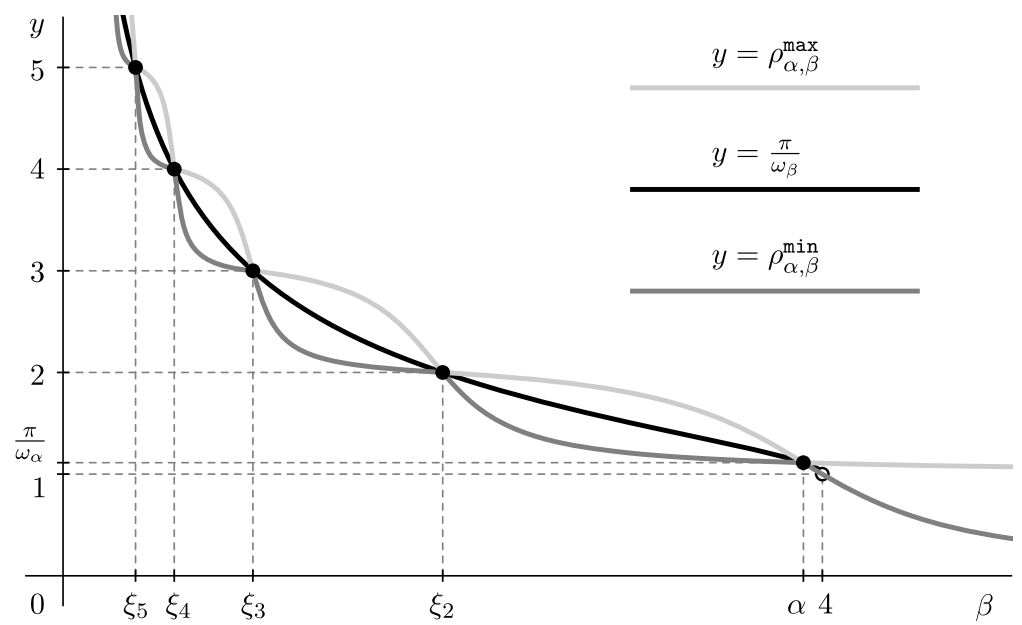

Fig. 7. Graphs of functions $\beta \mapsto \rho_{\alpha, \beta}^{\min }$ and $\beta \mapsto \rho_{\alpha, \beta}^{\max }$ for fixed $\alpha=3.9$ and the graph of the function $\beta \mapsto \frac{\pi}{\omega_{\beta}}$.

Let us recall that using $\tau_{\alpha, \beta}$, we can formulate an implicit description of the first non-trivial Fučík curve $\mathcal{C}_{1}^{ \pm}$as in (10) or (13). Now, using $\eta_{\alpha, \beta}$ and $\mu_{\alpha, \beta}$ in the following definition, let us introduce $\rho_{\alpha, \beta}^{\min }$ and $\rho_{\alpha, \beta}^{\max }$ that are given on the half-strip $\mathcal{D}$. See also Fig. 7 and note that $\rho_{\alpha, \beta}^{\min } \leq \frac{\pi}{\omega_{\beta}} \leq \rho_{\alpha, \beta}^{\max }$ for $0<\beta<4$.

Definition 2. For $0<\alpha<4$ and $\beta>0$, let us define

$$
\begin{array}{r}
\rho_{\alpha, \beta}^{\min }:= \begin{cases}2 \mu_{\alpha, \beta}+\kappa_{\beta} & \text { for } \alpha \leq \beta, \\
2 \eta_{\alpha, \beta}+\kappa_{\beta}+1 & \text { for } \alpha>\beta,\end{cases} \\
\rho_{\alpha, \beta}^{\max }:= \begin{cases}2 \eta_{\alpha, \beta}+\kappa_{\beta}+1 & \text { for } \alpha \leq \beta, \\
2 \mu_{\alpha, \beta}+\kappa_{\beta} & \text { for } \alpha>\beta .\end{cases}
\end{array}
$$

In the following theorem, we use $\rho_{\alpha, \beta}^{\min }$ and $\rho_{\alpha, \beta}^{\max }$ to construct sets $\Upsilon_{l}^{ \pm}$as bounds for Fučík curves $\mathcal{C}_{l}^{ \pm}$such that (see Figs. 9 and 8)

$$
\left(\mathcal{C}_{l}^{ \pm} \cap \mathcal{D}\right) \subset \Upsilon_{l}^{ \pm}
$$

Theorem 3. In the domain $\mathcal{D}=(0,4) \times(0,+\infty)$, we have the following bounds for Fučik curves $\mathcal{C}_{l}^{ \pm}, l=1, \ldots, n-1$,

$$
\begin{aligned}
\left(\mathcal{C}_{2 j-1}^{ \pm} \cap \mathcal{D}\right) & \subset \Upsilon_{j, j}=: \Upsilon_{2 j-1}^{ \pm} \\
\left(\mathcal{C}_{2 j}^{+} \cap \mathcal{D}\right) & \subset \Upsilon_{j+1, j}=: \Upsilon_{2 j}^{+} \\
\left(\mathcal{C}_{2 j}^{-} \cap \mathcal{D}\right) & \subset \Upsilon_{j, j+1}=: \Upsilon_{2 j}^{-}
\end{aligned}
$$

$j \in \mathbb{N}$, where for $k, s \in \mathbb{N}$, sets $\Upsilon_{k, s}$ are given by 

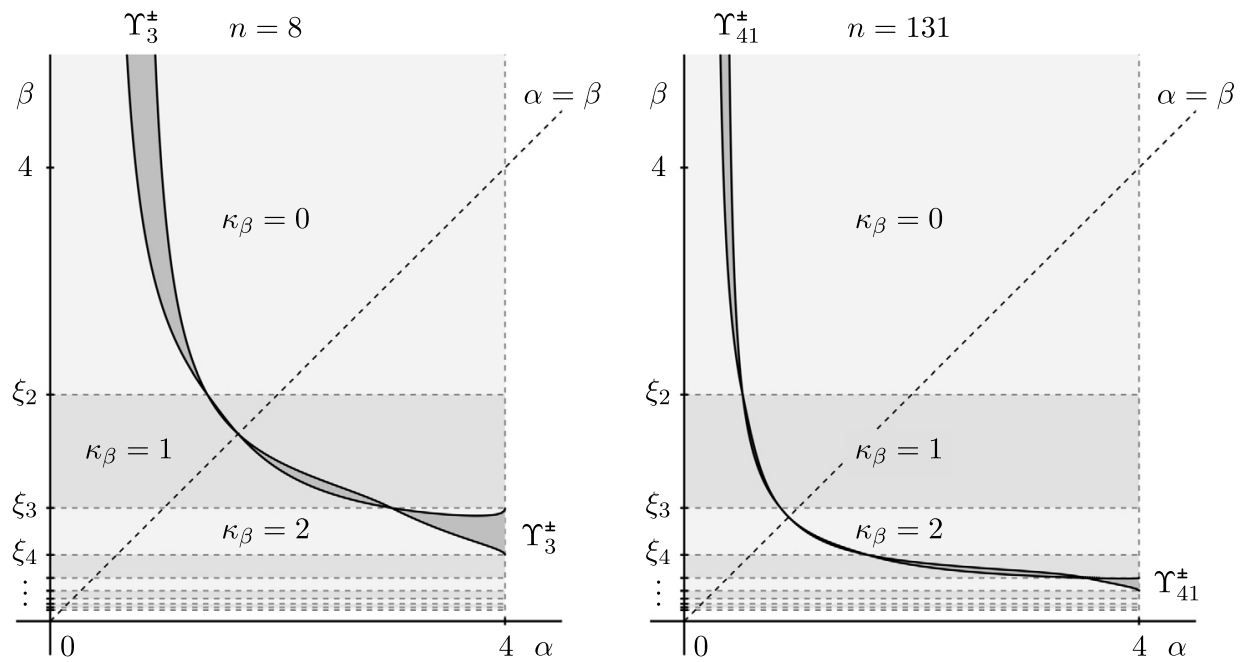

Fig. 8. The decomposition of the half-strip $\mathcal{D}$ into rectangles by $\kappa_{\beta}=k, k \in \mathbb{N}_{0}$, and the set $\Upsilon_{3}^{ \pm} \subset \mathcal{D}$ as a bound for the third non-trivial Fučík curve $\mathcal{C}_{3}^{ \pm}$for $n=8$ (left) and the set $\Upsilon_{41}^{ \pm} \subset \mathcal{D}$ as a bound for the forty-first non-trivial Fučík curve $\mathcal{C}_{41}^{ \pm}$for $n=131$ (right).

$$
\Upsilon_{k, s}:=\left\{(\alpha, \beta) \in \mathcal{D}: \rho_{\alpha, \beta}^{\min } \leq \frac{1}{s}\left(n+1-k \frac{\pi}{\omega_{\alpha}}\right) \leq \rho_{\alpha, \beta}^{\max }\right\}
$$

Remark 4. Due to Theorem 3, the part of the Fučík curve $\mathcal{C}_{2 j-1}^{ \pm}$that belongs to the half-strip $\mathcal{D}$ is in the set $\Upsilon_{2 j-1}^{ \pm}$with the boundary determined by two curves

$$
s\left(\kappa_{\beta}+2 \mu_{\alpha, \beta}\right)+k \frac{\pi}{\omega_{\alpha}}=n+1, \quad s\left(\kappa_{\beta}+2 \eta_{\alpha, \beta}+1\right)+k \frac{\pi}{\omega_{\alpha}}=n+1,
$$

where $k=s=j$. And similarly, parts of Fuč́́k curves $\mathcal{C}_{2 j}^{+} \cap \mathcal{D}$ and $\mathcal{C}_{2 j}^{-} \cap \mathcal{D}$ are in sets $\Upsilon_{2 j}^{+}$ and $\Upsilon_{2 j}^{-}$with boundaries given by curves in (21) for $k=j+1, s=j$ and $k=j, s=j+1$, respectively.

For $0<\alpha, \beta<4$, the equation (10), which describes the first non-trivial Fučík curve $\mathcal{C}_{1}^{ \pm}$, can be written in the following form

$$
\kappa_{\beta}+\tau_{\alpha, \beta}+1+\frac{\pi}{\omega_{\alpha}}=n+1,
$$

since $\left\lfloor\frac{\pi}{\omega_{\beta}}\right\rfloor=\kappa_{\beta}+1$ and $\left\lfloor\frac{\pi}{\omega_{\alpha}}\right\rfloor+\tau_{\alpha, \alpha}=\frac{\pi}{\omega_{\alpha}}$ (see Lemma 16). Let us note that the equation (22) has the same structure as equations in (21) which describe the boundary of the set $\Upsilon_{l}^{ \pm}$containing the particular Fučík curve $\mathcal{C}_{l}^{ \pm}\left(\tau_{\alpha, \beta}\right.$ is used in (22) instead of $\mu_{\alpha, \beta}$ or $\eta_{\alpha, \beta}$ in (21)). On the other hand, the structure of equations in (21) is much simpler than the known precise description of higher non-trivial Fučík curves $\mathcal{C}_{l}^{ \pm}$for $l \geq 2$. For example, compare (21) for $k=3$ and $s=2$ to the description of the fourth non-trivial Fučík curve $\mathcal{C}_{4}^{+}$which has the following form

$$
2\left\lfloor\frac{\pi}{\omega_{\alpha}}\right\rfloor+2\left\lfloor\tau_{\beta, \alpha}+\frac{\pi}{\omega_{\beta}}\right\rfloor+\left\lfloor\tau_{\alpha, \beta}^{2,+}+\frac{\pi}{\omega_{\alpha}}\right\rfloor+\tau_{\alpha, \beta}^{2,+}+\tau_{\alpha, \beta}^{3,+}=n+1,
$$



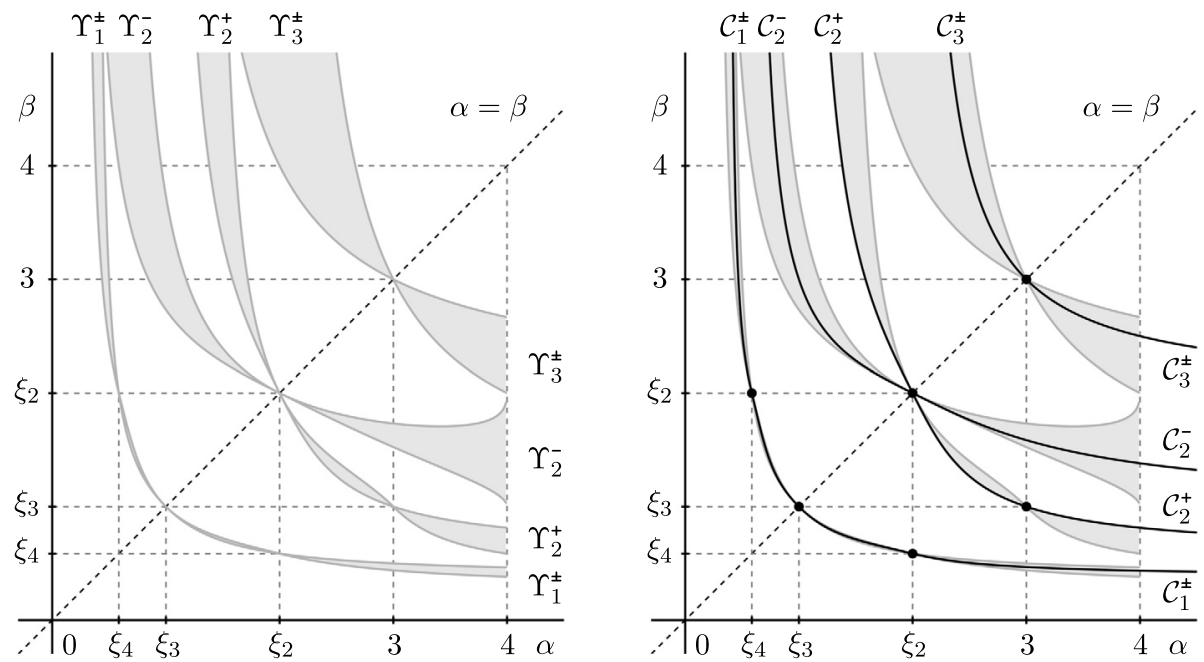

Fig. 9. Sets $\Upsilon_{l}^{ \pm}$in $\mathcal{D}$ (grey regions) as bounds for Fučík curves $\mathcal{C}_{l}^{ \pm}$(black curves, right) for $n=5$.

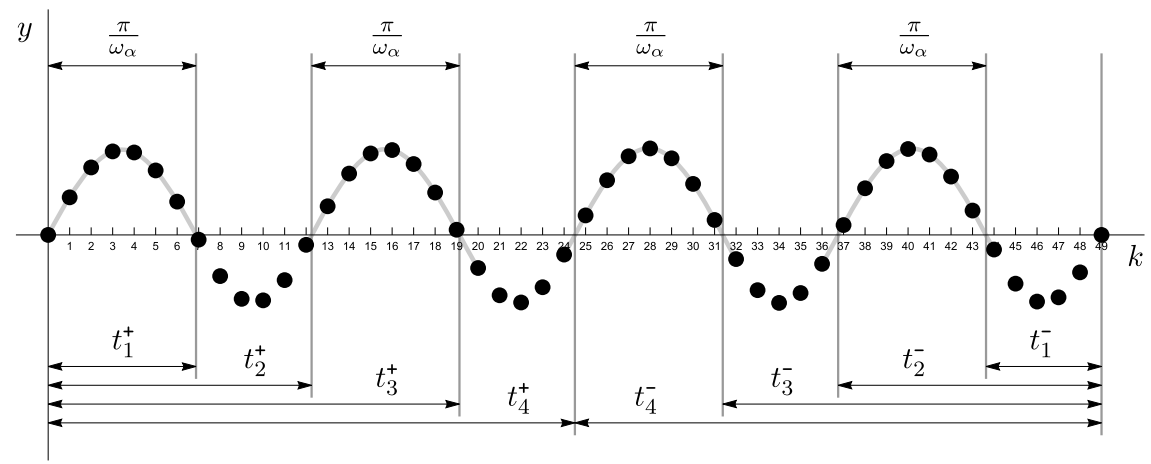

Fig. 10. A non-trivial solution of the problem (7) with 7 generalized zeros on $\mathbb{T}$ for $(\alpha, \beta) \in \mathcal{C}_{7}^{+}(n=48$, $\alpha \doteq 0.205, \beta \doteq 0.332)$.

where $\tau_{\alpha, \beta}^{2,+}$ and $\tau_{\alpha, \beta}^{3,+}$ are defined in (15) and (19), respectively.

The implicit description of all non-trivial Fučík curves $\mathcal{C}_{l}^{ \pm}$is provided in the next Theorem 5 . Let us note that $t_{j}^{+}$and $t_{j}^{-}$determine zeros of positive semi-waves (as continuous extensions) and $\rho_{\alpha, \beta}$ (introduced in Definition 19 in Section 5) measures the distance between every two consecutive zeros of two different positive semi-waves. See Fig. 10 and observe that $t_{1}^{+}=\frac{\pi}{\omega_{\alpha}}$ and $(\lceil\cdot\rceil$ denotes the ceil function $)$

$$
t_{2}^{+}=t_{1}^{+}+\rho_{\alpha, \beta}\left(\left\lceil t_{1}^{+}\right\rceil-t_{1}^{+}\right), \quad t_{3}^{+}=t_{2}^{+}+\frac{\pi}{\omega_{\alpha}}, \quad t_{4}^{+}=t_{3}^{+}+\rho_{\alpha, \beta}\left(\left\lceil t_{3}^{+}\right\rceil-t_{3}^{+}\right) .
$$

See also Figs. 29, 30 and 31 at the end of Section 5 for other examples of non-trivial solutions of the problem (7). 
Theorem 5. In the domain $\mathcal{D}=(0,4) \times(0,+\infty)$, we have the following description of Fučík curves $\mathcal{C}_{l}^{ \pm}, l=1, \ldots, n-1$,

$$
\begin{aligned}
& \mathcal{C}_{2 j-1}^{ \pm} \cap \mathcal{D}=\left\{(\alpha, \beta) \in \mathcal{D}: t_{j}^{+}(\alpha, \beta)+t_{j}^{-}(\alpha, \beta)=n+1\right\}, \\
& \mathcal{C}_{2 j}^{+} \cap \mathcal{D}=\left\{(\alpha, \beta) \in \mathcal{D}: t_{j+1}^{+}(\alpha, \beta)+t_{j}^{+}(\alpha, \beta)=n+1\right\}, \\
& \mathcal{C}_{2 j}^{-} \cap \mathcal{D}=\left\{(\alpha, \beta) \in \mathcal{D}: t_{j+1}^{-}(\alpha, \beta)+t_{j}^{-}(\alpha, \beta)=n+1\right\},
\end{aligned}
$$

where

$$
\begin{aligned}
t_{1}^{+}:=\frac{\pi}{\omega_{\alpha}}, \quad t_{j}^{+} & := \begin{cases}t_{j-1}^{+}+\rho_{\alpha, \beta}\left(\left\lceil t_{j-1}^{+}\right\rceil-t_{j-1}^{+}\right) & \text {for } j \text { even }, \\
t_{j-1}^{+}+\frac{\pi}{\omega_{\alpha}} & \text { for } j \text { odd },\end{cases} \\
t_{1}^{-}:=\rho_{\alpha, \beta}(0), \quad t_{j}^{-} & := \begin{cases}t_{j-1}^{-}+\frac{\pi}{\omega_{\alpha}} & \text { for } j \text { even }, \\
t_{j-1}^{-}+\rho_{\alpha, \beta}\left(\left\lceil t_{j-1}^{-}\right\rceil-t_{j-1}^{-}\right) & \text {for } j \text { odd } .\end{cases}
\end{aligned}
$$

Finally, let us point out that the value $\rho_{\alpha, \beta}(s)$ of the distance function $\rho_{\alpha, \beta}$ is bounded by $\rho_{\alpha, \beta}^{\min }$ and $\rho_{\alpha, \beta}^{\max }$ used in Theorem 3 to construct sets $\Upsilon_{l}^{ \pm}$as bounds for Fučík curves $\mathcal{C}_{l}^{ \pm}$.

\section{Connections between the Fučík spectra for discrete and continuous problems}

In this section, we show some consequences of obtained results in Theorems 3 and 5 in order to reveal the link between the Fučík spectrum $\Sigma$ for the discrete problem (7) and the Fučík spectrum $\Sigma^{\mathbf{c}}$ for the continuous problem (2). For this purpose, let us consider the following discrete Dirichlet problem

$$
\left\{\begin{array}{l}
\Delta_{h}^{2} v(k-h)+a v^{+}(k)-b v^{-}(k)=0, \quad k \in \mathbb{T}_{h} \\
u(0)=u(1)=0
\end{array}\right.
$$

where $a, b \in \mathbb{R}, h:=\frac{1}{n+1}, n \in \mathbb{N}, \mathbb{T}_{h}:=\{i h: i=1, \ldots, n\}$ and

$$
\Delta_{h}^{2} v(k-h)=\frac{v(k+h)-2 v(k)+v(k-h)}{h^{2}} .
$$

Thus, the problem (26) is the rescaled version of the original problem (7) and it can be also viewed as the result of a discretization of the continuous Dirichlet problem (2). The Fučík spectrum for the rescaled discrete problem (26) consists of finite number of Fučík curves $\mathcal{C}_{h, l}^{ \pm}, l=0, \ldots, n-1$, such that

$$
\mathcal{C}_{h, l}^{ \pm}=\left\{(a, b) \in \mathbb{R}^{2}:\left(a h^{2}, b h^{2}\right) \in \mathcal{C}_{l}^{ \pm}\right\}
$$



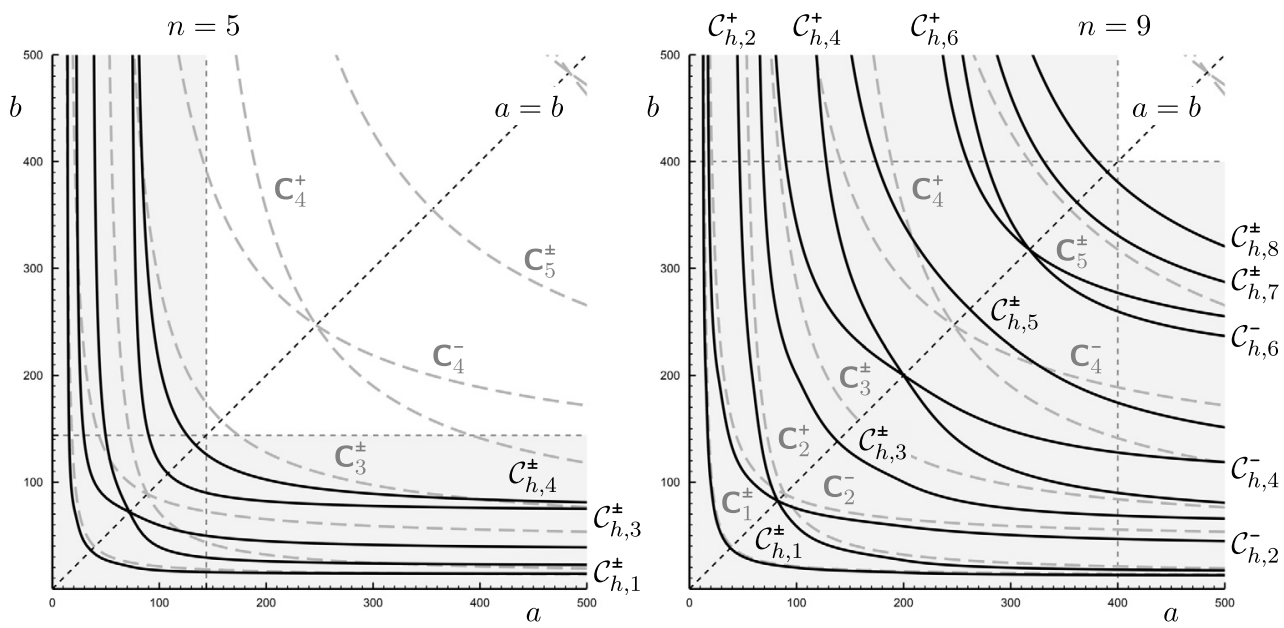

Fig. 11. Non-trivial Fučík curves $\mathbf{C}_{l}^{ \pm}$(grey dashed curves) for the continuous problem (2) and non-trivial Fučík curves $\mathcal{C}_{h, l}^{ \pm}$(black curves) for the rescaled discrete problem (26): five curves $\mathcal{C}_{h, 1}^{ \pm}, \mathcal{C}_{h, 2}^{+}, \mathcal{C}_{h, 2}^{-}, \mathcal{C}_{h, 3}^{ \pm}$and $\mathcal{C}_{h, 4}^{ \pm}$for $n=5$ in the domain $D_{h}=((0,144) \times(0,+\infty)) \cup((0,+\infty) \times(0,144))$ (grey region, left) and eleven curves $\mathcal{C}_{h, 1}^{ \pm}, \mathcal{C}_{h, 2}^{+}, \mathcal{C}_{h, 2}^{-}, \ldots, \mathcal{C}_{h, 8}^{ \pm}$for $n=9$ in the domain $D_{h}=((0,400) \times(0,+\infty)) \cup((0,+\infty) \times(0,400))$ (grey region, right).

where non-trivial Fučík curves $\mathcal{C}_{l}^{ \pm}$are described implicitly in Theorem 5. Since each nontrivial Fučík curve $\mathcal{C}_{l}^{ \pm}$is located in the domain $D=((0,4) \times(0,+\infty)) \cup((0,+\infty) \times(0,4))$ then each non-trivial Fučík curve $\mathcal{C}_{h, l}^{ \pm}$is contained in the domain

$$
D_{h}:=\left(\left(0,4 h^{-2}\right) \times(0,+\infty)\right) \cup\left((0,+\infty) \times\left(0,4 h^{-2}\right)\right) .
$$

See Fig. 11 for the domain $D_{h}$ containing all non-trivial Fučík curves $\mathcal{C}_{h, l}^{ \pm}$for the rescaled problem (26) and notice their correspondence to Fučík curves $\mathbf{C}_{l}^{ \pm}$for the continuous problem (2). Moreover, according to Theorem 3, we have for $l=1, \ldots, n-1$ that

$$
\left(\mathcal{C}_{h, l}^{ \pm} \cap \mathcal{D}_{h}\right) \subset \Upsilon_{h, l}^{ \pm}:=\left\{(a, b) \in \mathbb{R}^{2}:\left(a h^{2}, b h^{2}\right) \in \Upsilon_{l}^{ \pm}\right\}
$$

where $\mathcal{D}_{h}:=\left(0,4 h^{-2}\right) \times(0,+\infty)$. See Figs. 12 and 13 for sets $\Upsilon_{h, l}^{ \pm}$and check their correspondence to Fučík curves $\mathbf{C}_{l}^{ \pm}$for the continuous problem (2).

This paper is organized in the following way. Firstly, we recall some basic facts and results concerning mainly the semi-linear initial value problem in Section 4 . At the end of this section, in Theorem 13, we obtain some basic bounds for each non-trivial Fučík curve $\mathcal{C}_{l}^{ \pm}$using $\kappa_{\beta}$. The next Section 5 is devoted to the investigation of the distance $\rho_{\alpha, \beta}$ of two consecutive zeros of two different positive semi-waves as continuous extensions. We explore the properties of $\eta_{\alpha, \beta}, \tau_{\alpha, \beta}, \mu_{\alpha, \beta}$ and $\rho_{\alpha, \beta}$ in detail. This careful analysis leads to the proof of Theorem 5, which is available at the end of this section. The next Section 6 is devoted to the construction of improved bounds $\Upsilon_{l}^{ \pm}$for non-trivial Fučík curves $\mathcal{C}_{l}^{ \pm}$. In Theorem 31, we prove that $\rho_{\alpha, \beta}$ is a differentiable function which attains its global 


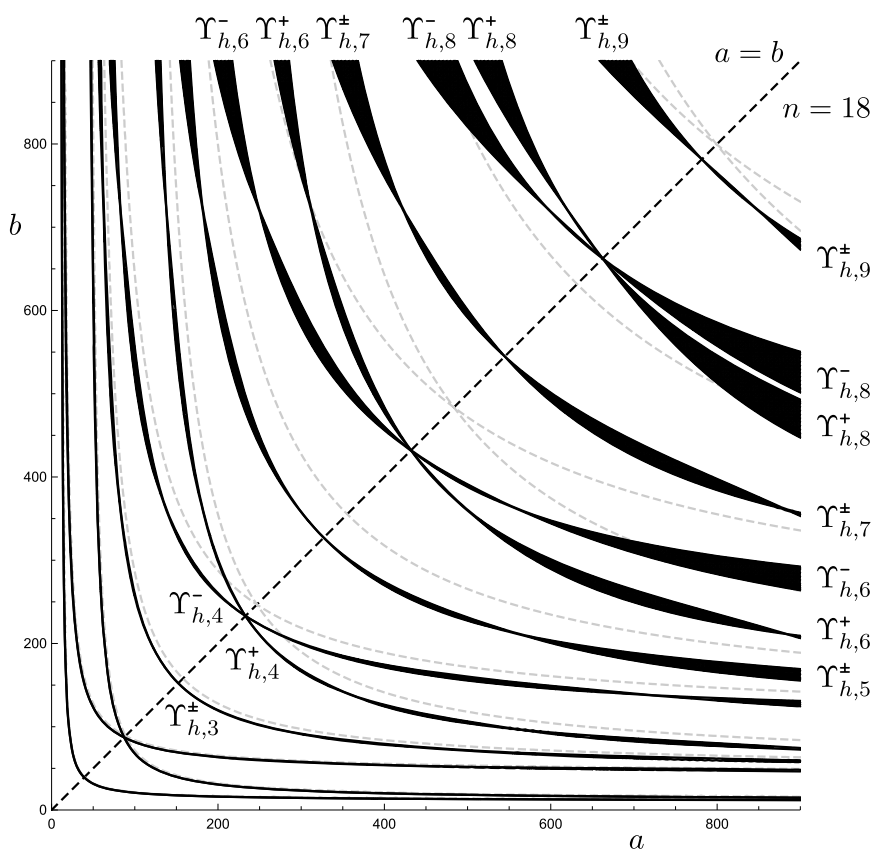

Fig. 12. Sets $\Upsilon_{h, l}^{ \pm}$(black regions), $l=1, \ldots, 9$, as bounds for Fučík curves $\mathcal{C}_{h, l}^{ \pm}$for the discrete rescaled problem (26) $(n=18)$ and Fučík curves $\mathbf{C}_{l}^{ \pm}$(grey dashed curves) for the continuous problem (2).

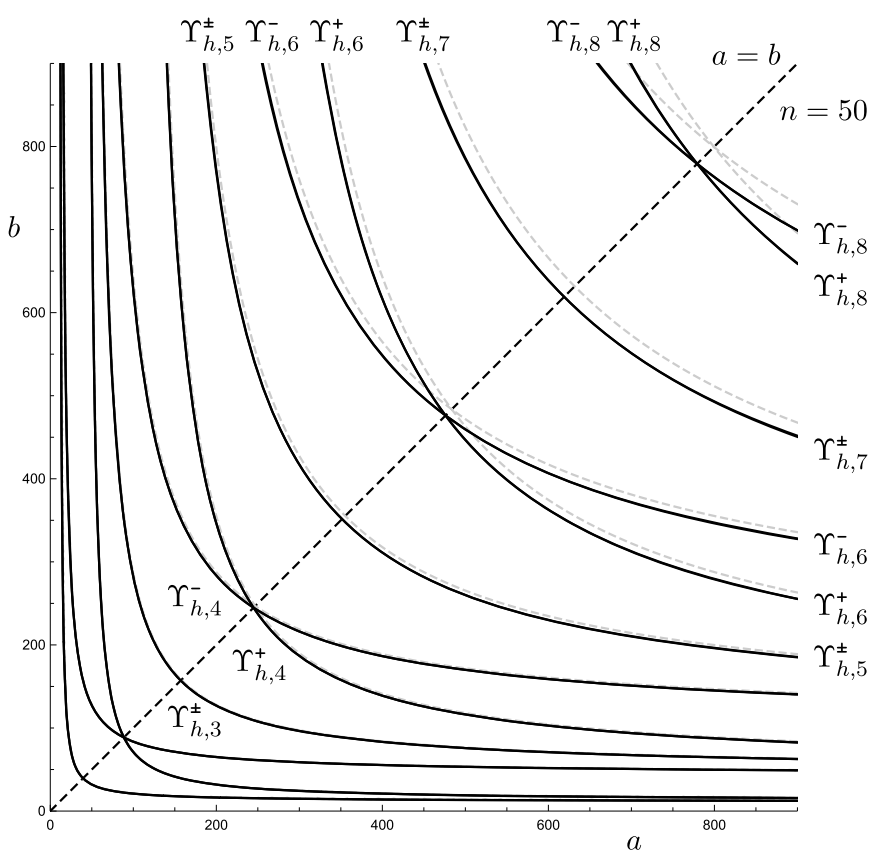

Fig. 13. Sets $\Upsilon_{h, l}^{ \pm}$(black thin regions), $l=1, \ldots, 8$, as bounds for Fučík curves $\mathcal{C}_{h, l}^{ \pm}$for the discrete rescaled problem (26) $(n=50)$ and Fuč́k curves $\mathbf{C}_{l}^{ \pm}$(grey dashed curves) for the continuous problem (2). 
extrema at points $\eta_{\alpha, \beta}$ and $\mu_{\alpha, \beta}$. Finally, the proof of the main Theorem 3 is available at the end of Section 6 and let us note that it is based on both Theorems 5 and 31 .

\section{Preliminaries and basic bounds for Fučík curves}

In the first part of this section, we recall some preliminaries used in [20], and we also prove some basic properties of $V_{\kappa_{\beta}}^{\beta}$ and $V_{\kappa_{\beta}+1}^{\beta}$ defined by Chebyshev polynomials of the second kind. Let us note that we follow the notation used in [20]. In the second part of this section, we deal with the sequence of functions $p_{i}$ introduced in [20] that are used to describe implicitly a non-trivial Fučík curve $\mathcal{C}_{l}^{ \pm}$(recall (10), (14) and (17), where $p_{1}$ and $p_{2}$ are used). Using $\kappa_{\beta}$, we provide a new description of functions $p_{i}$ in Lemma 14. Moreover, due to this description, we obtain some basic bounds for each non-trivial Fučík curve $\mathcal{C}_{l}^{ \pm}$in Theorem 13.

For $0<\alpha<4$, the function $T^{\alpha}$ defined in (12) is strictly increasing on $\mathbb{R}$ (see Fig. 14), $\operatorname{maps} \mathbb{R}^{*}$ onto $\left[0, \frac{\pi}{\omega_{\alpha}}\right)$ and

$$
T^{\alpha}(0)=1, \quad T^{\alpha}(-1)=\frac{1}{2}, \quad T^{\alpha}(1)=\frac{1}{2}+\frac{\pi}{2 \omega_{\alpha}}, \quad T^{\alpha}\left(\frac{2-\alpha}{2}\right)=\frac{\pi}{2 \omega_{\alpha}} .
$$

Moreover, we have the following useful formula (see Lemma 3 in [20])

$$
T^{\alpha}(q)+T^{\alpha}\left(\frac{1}{q}\right)=1 \quad \text { for } q \leq 0 \text { or } q=\infty
$$

Let us denote the inverse function of $T^{\alpha}$ by $Q^{\alpha}:\left[0, \frac{\pi}{\omega_{\alpha}}\right) \rightarrow \mathbb{R}^{*}$

$$
Q^{\alpha}(0)=\infty, \quad Q^{\alpha}(t)=-\frac{\sin \left(\omega_{\alpha}(1-t)\right)}{\sin \left(\omega_{\alpha} t\right)} \quad \text { for } 0<t<\frac{\pi}{\omega_{\alpha}}
$$

where $\omega_{\alpha}=\arccos \frac{2-\alpha}{2}$. Let us point out that $1<\frac{\pi}{\omega_{\alpha}}$ and that $Q^{\alpha}$ is a strictly increasing function on $\left(0, \frac{\pi}{\omega_{\alpha}}\right)$. Moreover, using (28), we obtain that

$$
Q^{\alpha}(t)=\frac{1}{Q^{\alpha}(1-t)} \quad \text { for } 0 \leq t \leq 1
$$

Let us consider the following semi-linear initial value problem

$$
\left\{\begin{array}{l}
\Delta^{2} u(k-1)+\alpha u^{+}(k)-\beta u^{-}(k)=0, \quad k \in \mathbb{Z}, \\
u(0)=0, \quad u(1)=C_{1}
\end{array}\right.
$$

where $C_{1} \in \mathbb{R}, C_{1} \neq 0$ and $(\alpha, \beta) \in D:=((0,4) \times(0,+\infty)) \cup((0,+\infty) \times(0,4))$. For $0<\alpha=\beta<4$, the problem (30) is a linear one and it has a unique solution $u$ of the form 


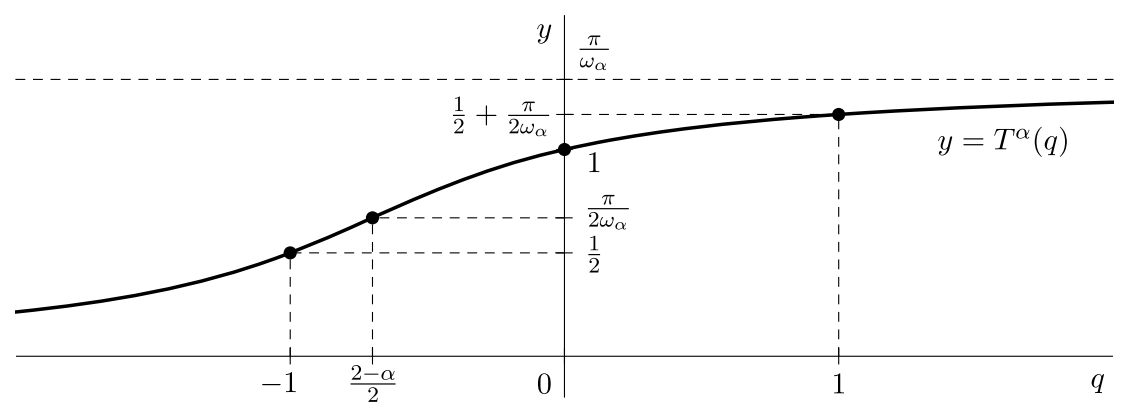

Fig. 14. The graph of the function $T^{\alpha}=T^{\alpha}(q)$ for fixed $\alpha=3.4$.

$$
u(k)=C_{1} \frac{\sin \left(\omega_{\beta} k\right)}{\sin \omega_{\beta}}=C_{1} V_{k-1}^{\beta},
$$

where $V_{k-1}^{\beta}$ is given in (8) by the Chebyshev polynomial of the second kind. For $(\alpha, \beta) \in$ $D$, the problem (30) has a unique solution $u$ which consists of infinitely many positive and negative semi-waves (as continuous extensions). Moreover, for $0<\alpha<4, \beta>0$ and $C_{1}>0$, we have due to Lemma 19 and Remark 20 in [20] that all non-negative zero points of all positive semi-waves form a sequence $\left(t_{j}\right)_{j=0}^{+\infty}$ such that

$$
t_{0}=0, \quad t_{j}=\left\{\begin{array}{ll}
\sum_{i=1}^{j} p_{i}(\alpha, \beta)+T^{\alpha}\left(\vartheta_{j}(\alpha, \beta)\right) & \text { for } C_{1}>0, \\
\sum_{i=1}^{j} p_{i}(\beta, \alpha)+T^{\alpha}\left(\vartheta_{j}(\beta, \alpha)\right) & \text { for } C_{1}<0,
\end{array} \quad j \in \mathbb{N},\right.
$$

where functions $p_{i}$ and $\vartheta_{i}$ are given recurrently for $i \in \mathbb{N}$ in the following way (see Definition 17 in [20])

$$
\begin{aligned}
\vartheta_{0}(\alpha, \beta) & :=\infty, \\
p_{2 i-1}(\alpha, \beta) & := \begin{cases}\left\lfloor T^{\alpha}\left(\vartheta_{2 i-2}(\alpha, \beta)\right)+\frac{\pi}{\omega_{\alpha}}\right\rfloor & \text { for } \alpha<4, \\
\left\lfloor T^{\beta}\left(\vartheta_{2 i-2}(\alpha, \beta)\right)+T^{\beta}(2-\alpha)+1\right\rfloor & \text { for } \alpha \geq 4,\end{cases} \\
p_{2 i}(\alpha, \beta) & := \begin{cases}\left\lfloor T^{\beta}\left(\vartheta_{2 i-1}(\alpha, \beta)\right)+\frac{\pi}{\omega_{\beta}}\right\rfloor & \text { for } \beta<4, \\
\left\lfloor T^{\alpha}\left(\vartheta_{2 i-1}(\alpha, \beta)\right)+T^{\alpha}(2-\beta)+1\right\rfloor & \text { for } \beta \geq 4,\end{cases} \\
\vartheta_{2 i-1}(\alpha, \beta) & :=W_{p_{2 i-1}(\alpha, \beta)}^{\alpha}\left(\vartheta_{2 i-2}(\alpha, \beta)\right), \\
\vartheta_{2 i}(\alpha, \beta): & W_{p_{2 i}(\alpha, \beta)}^{\beta}\left(\vartheta_{2 i-1}(\alpha, \beta)\right) .
\end{aligned}
$$

Finally, to complete the definition of $\vartheta_{i}$ in (34) and (35), let us recall the function $W_{k}^{\lambda}: \mathbb{R}^{*} \rightarrow \mathbb{R}^{*}$ for $\lambda \in \mathbb{R}$ and $k \in \mathbb{Z}$ as (see Definition 5 in [20]) 

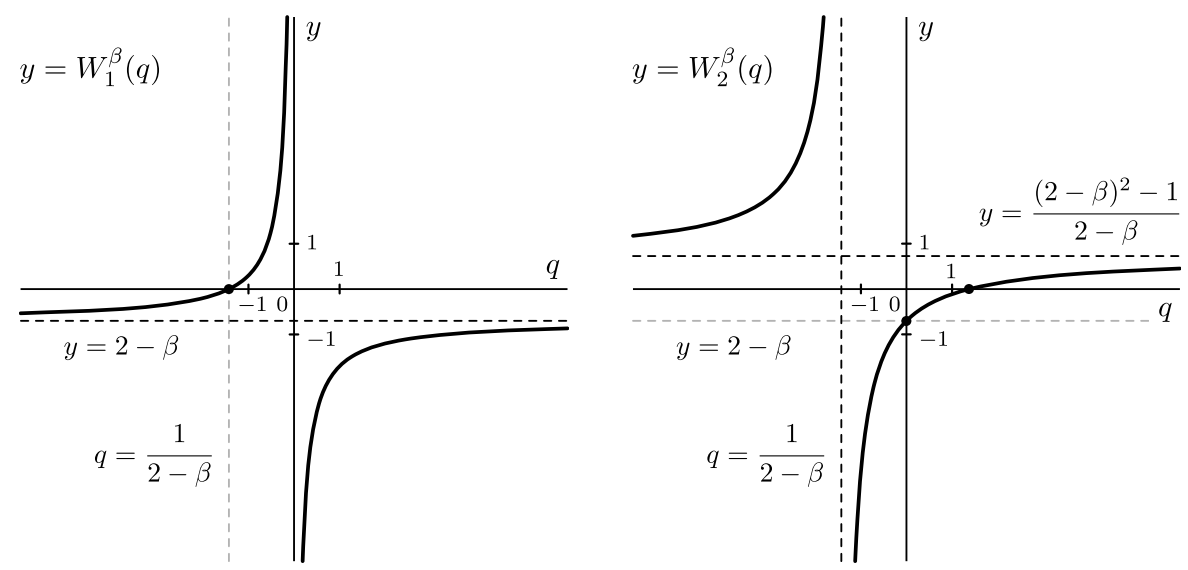

Fig. 15. Graphs of functions $W_{1}^{\beta}=W_{1}^{\beta}(q)$ (left) and $W_{2}^{\beta}=W_{2}^{\beta}(q)$ (right) for fixed $\beta=2.7$.

$$
W_{k}^{\lambda}(q):= \begin{cases}\frac{q \cdot V_{k}^{\lambda}-V_{k-1}^{\lambda}}{q \cdot V_{k-1}^{\lambda}-V_{k-2}^{\lambda}} & \text { for } q \in \mathbb{R}, \\ \frac{V_{k}^{\lambda}}{V_{k-1}^{\lambda}} & \text { for } q=\infty .\end{cases}
$$

Let us note that the function $W_{k}^{\lambda}$ is the restriction of a complex Möbius transformation on $\mathbb{R}^{*}$ (see Fig. 15). Now, let us recall some useful properties of $W_{k}^{\lambda}$ due to Lemma 9 in $[20]$

$$
W_{l}^{\lambda}\left(W_{k}^{\lambda}(q)\right)=W_{k+l}^{\lambda}(q), \quad W_{-k}^{\lambda}\left(W_{k}^{\lambda}(q)\right)=q, \quad W_{-k}^{\lambda}(q)=\frac{1}{W_{k}^{\lambda}\left(\frac{1}{q}\right)}
$$

where $k, l \in \mathbb{Z}$ and $q \in \mathbb{R}^{*}$. Moreover, due to Remark 10 in [20], we have for $\lambda \in \mathbb{R}$ and $k, l \in \mathbb{Z}$ that

$$
q_{k+l}=W_{l}^{\lambda}\left(q_{k}\right), \quad q_{k}:=\frac{u(k)}{u(k-1)},
$$

where $u$ is a non-trivial solution of the linear equation $\Delta^{2} u(k-1)+\lambda u(k)=0$.

In (36), the coefficients $V_{k}^{\lambda}$ are defined in (8) using Chebyshev polynomial of the second kind and thus, $V_{k}^{\lambda}$ satisfies the three terms recurrence formula

$$
V_{k-1}^{\lambda}-(2-\lambda) V_{k}^{\lambda}+V_{k+1}^{\lambda}=0
$$

Moreover, by Lemma 4 in [20], we also have

$$
\left(V_{k}^{\lambda}\right)^{2}-V_{k+1}^{\lambda} V_{k-1}^{\lambda}=1
$$

Let us introduce the next identity for Chebyshev polynomials of the second kind. 


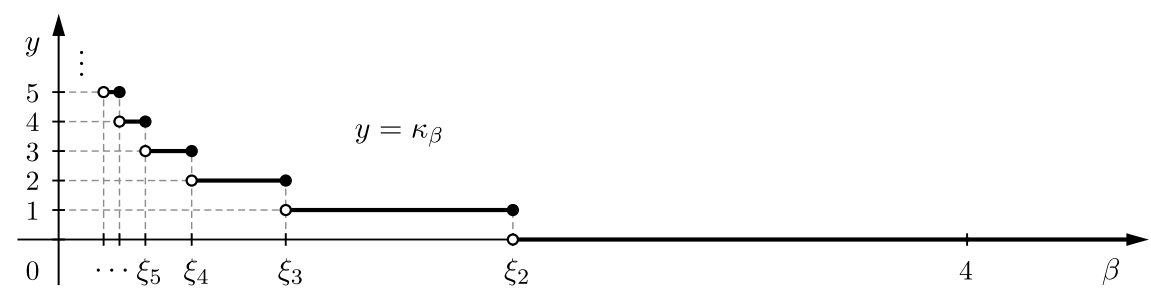

Fig. 16. The graph of the piecewise constant function $\beta \mapsto \kappa_{\beta}$.

Lemma 6. For $\lambda \in \mathbb{R}$ and $k \in \mathbb{Z}$, the following equality holds

$$
\left(V_{k+1}^{\lambda}-V_{k}^{\lambda}\right)^{2}=1-\lambda \cdot V_{k+1}^{\lambda} V_{k}^{\lambda}
$$

Proof. Using (39) and (40), we obtain

$$
\begin{aligned}
\left(V_{k+1}^{\lambda}-V_{k}^{\lambda}\right)^{2} & =\left(V_{k+1}^{\lambda}\right)^{2}-2 V_{k+1}^{\lambda} V_{k}^{\lambda}+\left(V_{k}^{\lambda}\right)^{2}+\lambda V_{k+1}^{\lambda} V_{k}^{\lambda}-\lambda V_{k+1}^{\lambda} V_{k}^{\lambda} \\
& =\left(V_{k+1}^{\lambda}\right)^{2}-V_{k+1}^{\lambda}(2-\lambda) V_{k}^{\lambda}+\left(V_{k}^{\lambda}\right)^{2}-\lambda V_{k+1}^{\lambda} V_{k}^{\lambda} \\
& =\left(V_{k+1}^{\lambda}\right)^{2}-V_{k+1}^{\lambda}\left(V_{k-1}^{\lambda}+V_{k+1}^{\lambda}\right)+1+V_{k+1}^{\lambda} V_{k-1}^{\lambda}-\lambda V_{k+1}^{\lambda} V_{k}^{\lambda} \\
& =1-\lambda \cdot V_{k+1}^{\lambda} V_{k}^{\lambda} .
\end{aligned}
$$

Now, let us take into account $\kappa_{\beta}$ defined in (20) for $\beta>0$ (see Fig. 16). The function $\beta \mapsto \kappa_{\beta}$ is a piecewise constant and decreasing function, which has a jump discontinuity at $\xi_{k}$ for $k \in \mathbb{N}, k \geq 2$, defined in (9). Let us note that for $\beta=\xi_{k}, k \in \mathbb{N}, k \geq 2$, we have $\omega_{\beta}=\frac{\pi}{k}, \kappa_{\beta}=k-1$ and $W_{k}^{\beta}$ is the identity function on $\mathbb{R}^{*}$ (see Lemma 11 in [20] for $\lambda=\beta$ and $j=1$ ). Thus, we have

$$
W_{\kappa_{\beta}+1}^{\beta}(q)=q, \quad q \in \mathbb{R}^{*}, \quad \text { for } \beta=\xi_{k}, k \in \mathbb{N}, k \geq 2 .
$$

Let us investigate some basic properties of $V_{\kappa_{\beta}}^{\beta}$ and $V_{\kappa_{\beta}+1}^{\beta}$ (see Figs. 18 and 19).

Lemma 7. For $\beta>0$, we have $0 \leq V_{\kappa_{\beta}}^{\beta} \leq 1$ and $V_{\kappa_{\beta}+1}^{\beta}<0$. Moreover, $V_{\kappa_{\beta}}^{\beta}$ and $V_{\kappa_{\beta}+1}^{\beta}$ have the following properties:

1. $V_{\kappa_{\beta}}^{\beta}=1$ if and only if $\beta>\xi_{2}=2$.

2. $V_{\kappa_{\beta}}^{\beta}=0$ if and only if $\beta=\xi_{k}$ for some $k \in \mathbb{N}, k \geq 2$.

3. $V_{\kappa_{\beta}+1}^{\beta}=-1$ for $\beta=\xi_{k}, k \in \mathbb{N}, k \geq 2$.

4. If $V_{\kappa_{\beta}}^{\beta}+V_{\kappa_{\beta}+1}^{\beta}=-1$ for $0<\beta \neq 4$ then $\beta=\xi_{k}$ for some $k \in \mathbb{N}, k \geq 2$.

Proof. At first, let us assume that $\beta>\xi_{2}=2$. In this case, we have $\kappa_{\beta}=0$ and thus $V_{\kappa_{\beta}}^{\beta}=V_{0}^{\beta} \equiv 1$ and $V_{\kappa_{\beta}+1}^{\beta}=V_{1}^{\beta}=2-\beta<0$.

At second, let us assume that $\xi_{k+2}<\beta \leq \xi_{k+1} \leq \xi_{2}$ for fixed $k \in \mathbb{N}$. Then $\kappa_{\beta}=k$ and it suffices to show that (see Fig. 17) 


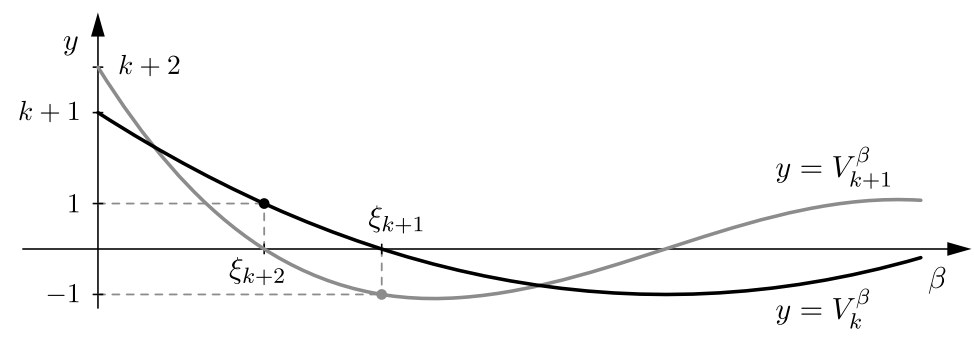

Fig. 17. Graphs of functions $\beta \mapsto V_{k}^{\beta}$ (black curve) and $\beta \mapsto V_{k+1}^{\beta}$ (grey curve).

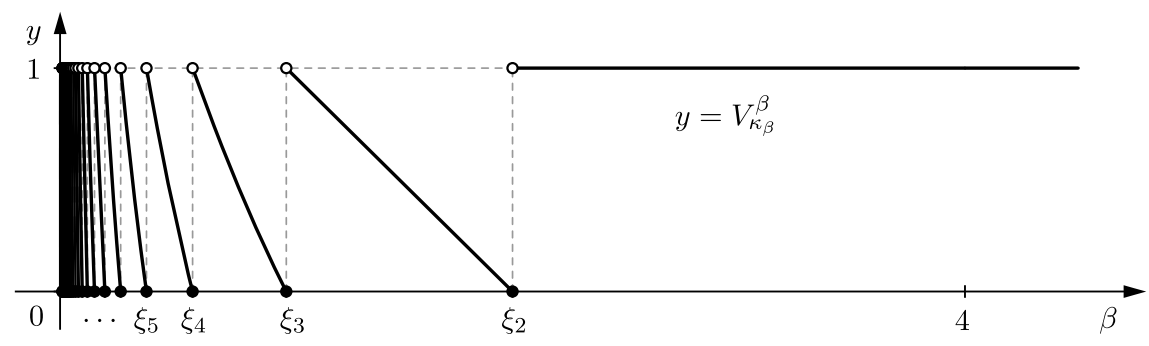

Fig. 18. The graph of the function $\beta \mapsto V_{\kappa_{\beta}}^{\beta}$.

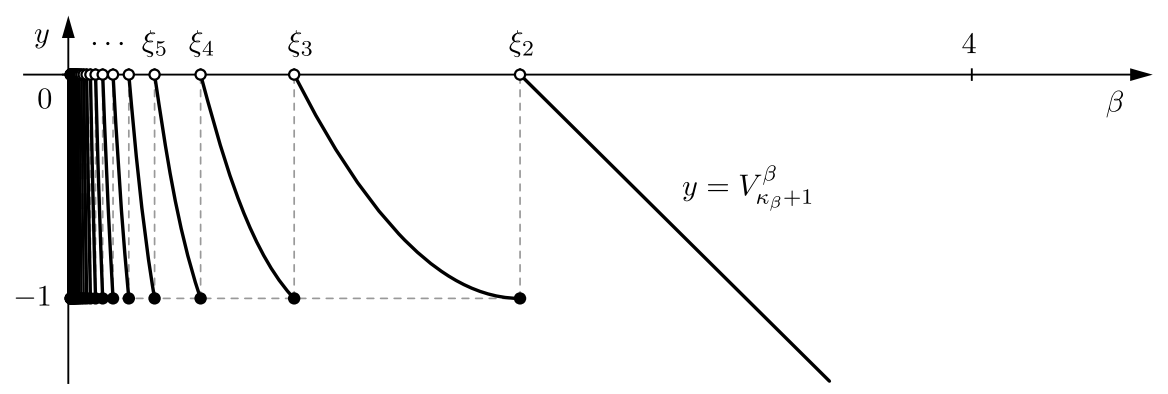

Fig. 19. The graph of the function $\beta \mapsto V_{\kappa_{\beta}+1}^{\beta}$.

1. $V_{k}^{\beta}=0$ and $V_{k+1}^{\beta}=-1$ for $\beta=\xi_{k+1}$,

2. $0<V_{k}^{\beta}<1$ and $V_{k+1}^{\beta}<0$ for $\xi_{k+2}<\beta<\xi_{k+1}$.

Now, we have that $V_{k}^{\beta}=0$ if and only if $\beta=4 \sin ^{2} \frac{m \pi}{2(k+1)}, m \in\{1, \ldots, k\}$. Thus, the first zero of $V_{k}^{\beta}$ is $\beta=\xi_{k+1}$. Similarly, $\beta=\xi_{k+2}<\xi_{k+1}$ is the first zero of $V_{k+1}^{\beta}$. Moreover, we have that $V_{k}^{\beta}>0$ for $0<\beta<\xi_{k+1}$ since for $\beta=0$, we have $V_{k}^{\beta}=k+1>0$. Using (41) for $\lambda=\beta=\xi_{k+2}$, we obtain

$$
\left(V_{k+1}^{\beta}-V_{k}^{\beta}\right)^{2}+\xi_{k+2} \cdot V_{k+1}^{\beta} V_{k}^{\beta}=1,
$$


which simplifies to $\left(V_{k}^{\beta}\right)^{2}=1$ since $V_{k+1}^{\beta}=0$ for $\beta=\xi_{k+2}$. Thus, we conclude that $V_{k}^{\beta}=1$ for $\beta=\xi_{k+2}<\xi_{k+1}$ since $V_{k}^{\beta}$ is positive for $0<\beta<\xi_{k+1}$. The Chebyshev polynomial of the second kind monotonically oscillates between its extrema and the first extreme of $\beta \mapsto V_{k}^{\beta}$ does not belong to the interval $\left(0, \xi_{k+1}\right)$. Thus, for $\xi_{k+2}<\beta<\xi_{k+1}$, we have that $0<V_{k}^{\beta}<1$. Since Chebyshev polynomials of the second kind are orthogonal with weight function $\omega(x)=\sqrt{1-x^{2}}$, using Corollary 3.3.3 on page 93 in [19], we have that two consecutive polynomials strictly interlace, i.e. between two consecutive zeros of $V_{k+1}^{\beta}$ is exactly one zero of $V_{k}^{\beta}$. Since $V_{k+1}^{\beta}=0$ for $\beta=\xi_{k+2}$ and $V_{k}^{\beta}=0$ for $\beta=\xi_{k+1}$, we have that $V_{k+1}^{\beta}<0$ for $\xi_{k+2}<\beta<\xi_{k+1}$. Finally, using (41) for $\lambda=\beta=\xi_{k+1}$, we obtain $\left(V_{k+1}^{\beta}\right)^{2}=1$ and thus, we conclude that $V_{k+1}^{\beta}=-1$.

Now, it remains to justify the last statement. Thus, let us assume that $V_{\kappa_{\beta}}^{\beta}+V_{\kappa_{\beta}+1}^{\beta}=$ -1 for $0<\beta \neq 4$. Using (41) for $\lambda=\beta$ and $k=\kappa_{\beta}$, we obtain

$$
\begin{aligned}
\left(V_{\kappa_{\beta}+1}^{\beta}-V_{\kappa_{\beta}}^{\beta}\right)^{2}+\beta \cdot V_{\kappa_{\beta}+1}^{\beta} V_{\kappa_{\beta}}^{\beta} & =1, \\
\left(2 V_{\kappa_{\beta}}^{\beta}+1\right)^{2}-\beta \cdot\left(V_{\kappa_{\beta}}^{\beta}+1\right) V_{\kappa_{\beta}}^{\beta} & =1, \\
4\left(V_{\kappa_{\beta}}^{\beta}\right)^{2}+4 V_{\kappa_{\beta}}^{\beta}-\beta \cdot\left(V_{\kappa_{\beta}}^{\beta}\right)^{2}-\beta \cdot V_{\kappa_{\beta}}^{\beta} & =0, \\
(4-\beta)\left(V_{\kappa_{\beta}}^{\beta}+1\right) V_{\kappa_{\beta}}^{\beta} & =0, \\
(\beta-4) V_{\kappa_{\beta}+1}^{\beta} V_{\kappa_{\beta}}^{\beta} & =0,
\end{aligned}
$$

which implies that $V_{\kappa_{\beta}}^{\beta}=0$ and thus, $\beta=\xi_{k}$ for some $k \in \mathbb{N}, k \geq 2$.

In the second part of this section, we simplify the definition of functions $p_{i}=p_{i}(\alpha, \beta)$ given by (32) and (33) within the following four lemmas. As a consequence of this simplification, we also obtain the basic bounds for each non-trivial Fučík curve $\mathcal{C}_{l}^{ \pm}$.

Lemma 8. For $0<\beta<4$, we have

$$
\frac{\pi}{\omega_{\beta}}-\left\lfloor\frac{\pi}{\omega_{\beta}}\right\rfloor=T^{\beta}\left(W_{\kappa_{\beta}+1}^{\beta}(\infty)\right)
$$

Proof. We have that $\frac{\pi}{\omega_{\beta}}-\left\lfloor\frac{\pi}{\omega_{\beta}}\right\rfloor=\frac{\pi}{\omega_{\beta}}-1-\kappa_{\beta}$ and thus, using (31), we get

$$
\begin{aligned}
Q^{\beta}\left(\frac{\pi}{\omega_{\beta}}-1-\kappa_{\beta}\right) & =-\frac{\sin \left(\omega_{\beta}\left(1-\frac{\pi}{\omega_{\beta}}+1+\kappa_{\beta}\right)\right)}{\sin \left(\omega_{\beta}\left(\frac{\pi}{\omega_{\beta}}-1-\kappa_{\beta}\right)\right)}=\frac{\sin \left(\omega_{\beta}\left(\kappa_{\beta}+2\right)\right)}{\sin \left(\omega_{\beta}\left(\kappa_{\beta}+1\right)\right)} \\
& =\frac{V_{\kappa_{\beta}+1}^{\beta}}{V_{\kappa_{\beta}}^{\beta}}=W_{\kappa_{\beta}+1}^{\beta}(\infty) .
\end{aligned}
$$




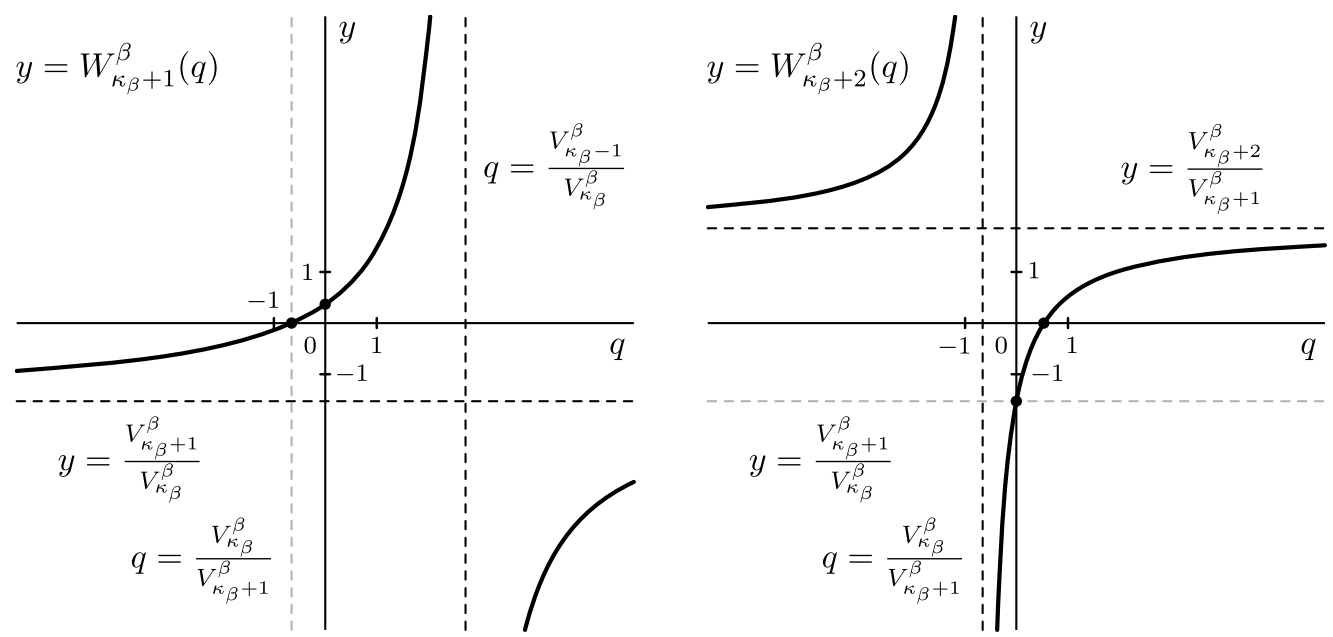

Fig. 20. Graphs of functions $W_{\kappa_{\beta}+1}^{\beta}=W_{\kappa_{\beta}+1}^{\beta}(q)$ (left) and $W_{\kappa_{\beta}+2}^{\beta}=W_{\kappa_{\beta}+2}^{\beta}(q)$ (right) for fixed $\beta=0.8$ (i.e. $\left.\kappa_{\beta}=2\right)$.

Lemma 9. Let $\beta>0$.

1. If $\beta=\xi_{k}, k \in \mathbb{N}, k \geq 2$, then $W_{\kappa_{\beta}+1}^{\beta}(q)=\infty$ if and only if $q=\infty$.

2. If $\beta>2$ then $W_{\kappa_{\beta}+1}^{\beta}(q)=\infty$ if and only if $q=0$.

3. If $\beta<2$ and $\beta \neq \xi_{k}, k \in \mathbb{N}, k \geq 2$, then $W_{\kappa_{\beta}+1}^{\beta}(q)$ is finite for $q \leq 0$ and for $q=\infty$.

Proof. Firstly, in the case of $\beta=\xi_{k}, k \in \mathbb{N}, k \geq 2$, we have $k=\kappa_{\beta}+1$ and $W_{\kappa_{\beta}+1}^{\beta}$ is the identity function (recall (42)). Secondly, for $\beta>2$, we have $\kappa_{\beta}=0$ and $W_{\kappa_{\beta}+1}^{\beta}(q)=$ $W_{1}^{\beta}(q)=2-\beta-1 / q$ (see Fig. 15). Thirdly, let us assume that $\beta \neq \xi_{k}, k \in \mathbb{N}, k \geq 2$, and that $0<\beta<2$. Then we have (see Fig. 20)

$$
W_{\kappa_{\beta}+1}^{\beta}(q)= \begin{cases}\frac{q \cdot V_{\kappa_{\beta}+1}^{\beta}-V_{\kappa_{\beta}}^{\beta}}{q \cdot V_{\kappa_{\beta}}^{\beta}-V_{\kappa_{\beta}-1}^{\beta}} & \text { for } q \in \mathbb{R}, \\ \frac{V_{\kappa_{\beta}+1}^{\beta}}{V_{\kappa_{\beta}}^{\beta}} & \text { for } q=\infty .\end{cases}
$$

Using Lemma 7 , we obtain that $W_{\kappa_{\beta}+1}^{\beta}(\infty)$ is negative and that $W_{\kappa_{\beta}+1}^{\beta}(q)$ is finite for $q \leq 0$. Indeed, using (40) for $\lambda=\beta$ and $k=\kappa_{\beta}$, we have

$$
q \cdot V_{\kappa_{\beta}}^{\beta}-V_{\kappa_{\beta}-1}^{\beta}=\frac{q \cdot V_{\kappa_{\beta}}^{\beta} V_{\kappa_{\beta}+1}^{\beta}+1-\left(V_{\kappa_{\beta}}^{\beta}\right)^{2}}{V_{\kappa_{\beta}+1}^{\beta}}<0 .
$$

The following lemma is based on Lemmas 14 and 16 in [20] and it allows us to determine the length of the interval $[i-1, j+1]$ for a positive or negative semi-wave 

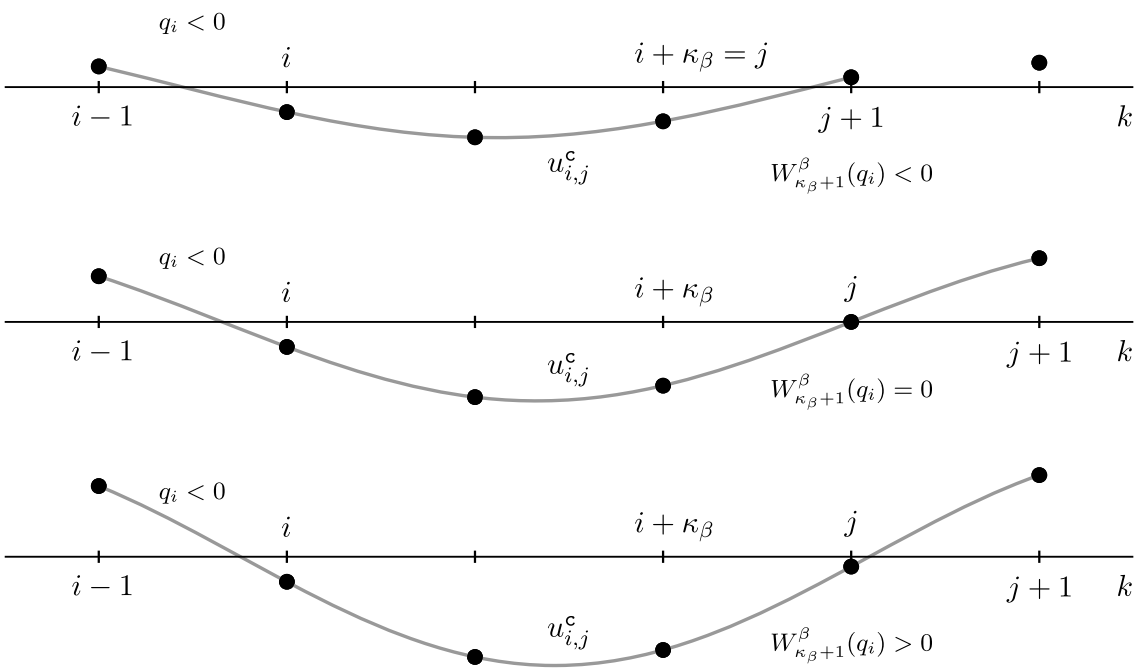

Fig. 21. The length of the interval $[i-1, j+1]$ for a negative semi-wave $u_{i, j}^{\mathrm{c}}$ of the solution $u$ of (30) for fixed $(\alpha, \beta) \in D$ according to the sign of $W_{\kappa_{\beta}+1}^{\beta}\left(q_{i}\right): j=i+\kappa_{\beta}+1$ and $u(j)<0$ (bottom), $j=i+\kappa_{\beta}+1$ and $u(j)=0$ (middle) and $j=i+\kappa_{\beta}$ and $u(j)<0$ (top).

of the solution $u$ according to the ratio $q_{i}$ of the values $u(i)$ and $u(i-1)$ (see Fig. 21). Let us note that conditions in (45) or (46) mean that the solution $u$ has a positive or negative semi-wave on the interval $[i-1, j+1]$.

Lemma 10. Let $(\alpha, \beta) \in D$ and $u$ be the solution of the initial value problem (30). Moreover, let $i, j \in \mathbb{Z}$ be such that $i \leq j$ and

$$
u(i-1)<0, \quad u(k) \geq 0 \quad \text { for } k=i, \ldots, j, \quad u(j+1)<0,
$$

or

$$
u(i-1)>0, \quad u(k) \leq 0 \quad \text { for } k=i, \ldots, j, \quad u(j+1)>0 .
$$

Then we have

$$
j=\left\{\begin{array}{lll}
i+\kappa_{\lambda} & \text { for } & W_{\kappa_{\lambda}+1}^{\lambda}\left(q_{i}\right)<0 \\
i+\kappa_{\lambda}+1 & \text { for } & W_{\kappa_{\lambda}+1}^{\lambda}\left(q_{i}\right) \geq 0
\end{array}\right.
$$

where we denoted $q_{i}:=\frac{u(i)}{u(i-1)} \leq 0$ and $\lambda=\alpha$ if (45) holds or $\lambda=\beta$ if (46) holds. Moreover, we have $u(k) \neq 0$ for $k \in \mathbb{Z}$ such that $i<k<j$, and $u(j)=0$ if and only if $W_{\kappa_{\lambda}+1}^{\lambda}\left(q_{i}\right)=0$.

Proof. Let us assume that conditions in (46) hold, which means that we have a negative semi-wave $u_{i, j}^{\mathrm{c}}$ of $u$ defined on the interval $[i-1, j+1]$ (see Fig. 21). Moreover, let us 
assume that the value $W_{\kappa_{\beta}+1}^{\beta}\left(q_{i}\right)$ is finite and we split the proof according to the value of $\beta$.

At first, let us consider $0<\beta<4$. Using Lemma 14 in [20], we have that

$$
j=i+\left\lfloor T^{\beta}\left(q_{i}\right)+\frac{\pi}{\omega_{\beta}}\right\rfloor-1
$$

where $q_{i}=\frac{u(i)}{u(i-1)} \leq 0$. If we denote $s=1-T^{\beta}\left(q_{i}\right)$ then (48) reads

$$
j=i+\left\lfloor\frac{\pi}{\omega_{\beta}}-s\right\rfloor
$$

and $s \in[0,1)$ since $0<T^{\beta}\left(q_{i}\right) \leq 1$. Now, let us consider that

$$
s>\frac{\pi}{\omega_{\beta}}-\left\lfloor\frac{\pi}{\omega_{\beta}}\right\rfloor \geq 0
$$

which implies that $\left\lfloor\frac{\pi}{\omega_{\beta}}\right\rfloor-1 \leq \frac{\pi}{\omega_{\beta}}-1<\frac{\pi}{\omega_{\beta}}-s<\left\lfloor\frac{\pi}{\omega_{\beta}}\right\rfloor$ and that $\left\lfloor\frac{\pi}{\omega_{\beta}}-s\right\rfloor=\left\lfloor\frac{\pi}{\omega_{\beta}}\right\rfloor-1=$ $\kappa_{\beta}$. Thus, we obtain using (49) that

$$
j=i+\kappa_{\beta} .
$$

Moreover, using (43), (27) and (37), the strict inequality in (50) reads

$$
\begin{aligned}
1-T^{\beta}\left(q_{i}\right) & >T^{\beta}\left(W_{\kappa_{\beta}+1}^{\beta}(\infty)\right), \\
T^{\beta}\left(q_{i}\right) & <T^{\beta}\left(W_{-\left(\kappa_{\beta}+1\right)}^{\beta}(0)\right), \\
W_{\kappa_{\beta}+1}^{\beta}\left(q_{i}\right) & <0,
\end{aligned}
$$

which justifies (47) if we take into account (51). Now, let us consider that

$$
0 \leq s \leq \frac{\pi}{\omega_{\beta}}-\left\lfloor\frac{\pi}{\omega_{\beta}}\right\rfloor
$$

which implies $\left\lfloor\frac{\pi}{\omega_{\beta}}\right\rfloor \leq \frac{\pi}{\omega_{\beta}}-s \leq \frac{\pi}{\omega_{\beta}}<\left\lfloor\frac{\pi}{\omega_{\beta}}\right\rfloor+1$ and that $\left\lfloor\frac{\pi}{\omega_{\beta}}-s\right\rfloor=\left\lfloor\frac{\pi}{\omega_{\beta}}\right\rfloor=\kappa_{\beta}+1$. Thus, we obtain using (49) that

$$
j=i+\kappa_{\beta}+1
$$

And similarly as in the previous case, using (43), (27) and (37), the second inequality in (52) reads $W_{\kappa_{\beta}+1}^{\beta}\left(q_{i}\right) \geq 0$, which justifies (47) if we take into account (53).

At second, let us consider $\beta \geq 4$. Then we have $0<\alpha<4$ and using Lemma 16 in [20], we obtain that

$$
j=i+\left\lfloor T^{\alpha}\left(q_{i}\right)+T^{\alpha}(2-\beta)\right\rfloor,
$$


where $q_{i}=\frac{u(i)}{u(i-1)} \leq 0$. Since $0<T^{\alpha}\left(q_{i}\right) \leq 1$ and $0<T^{\alpha}(2-\beta)<\frac{1}{2}$, we have $0<T^{\alpha}\left(q_{i}\right)+T^{\alpha}(2-\beta)<\frac{3}{2}$ and thus, (54) reads

$$
\begin{aligned}
j=i & \text { for } \quad T^{\alpha}\left(q_{i}\right)+T^{\alpha}(2-\beta)<1, \\
j=i+1 & \text { for } \quad T^{\alpha}\left(q_{i}\right)+T^{\alpha}(2-\beta) \geq 1 .
\end{aligned}
$$

The inequality in $(55)$ reads $T^{\alpha}\left(q_{i}\right)<T^{\alpha}\left(\frac{1}{2-\beta}\right)$ or $q_{i}<W_{-1}^{\beta}(0)$, which justifies (47) since $\kappa_{\beta}=0$ for $\beta \geq 4$. And similarly, (56) can be identified with the second case in (47).

Finally, for $\beta>0$ and $k \in \mathbb{Z}$ such that $i<k<j$, we have that $u(k)<0$. In contrary, if we assume that $u(k)=0$ for some $k$ strictly between $i$ and $j$ then $u(k-1) u(k+1)<0$, which contradicts (46). Moreover, we have $u(j)=0$ if and only if $W_{\kappa_{\beta}+1}^{\beta}\left(q_{i}\right)=0$. Indeed, using (38), we have

$$
W_{\kappa_{\beta}+1}^{\beta}\left(q_{i}\right)=q_{i+\kappa_{\beta}+1}= \begin{cases}q_{j+1}=\frac{u(j+1)}{u(j)} & \text { for } W_{\kappa_{\beta}+1}^{\beta}\left(q_{i}\right)<0 \\ q_{j}=\frac{u(j)}{u(j-1)} & \text { for } W_{\kappa_{\beta}+1}^{\beta}\left(q_{i}\right) \geq 0\end{cases}
$$

Thus, the proof is complete in the case of a negative semi-wave such that the value $W_{\kappa_{\beta}+1}^{\beta}\left(q_{i}\right)$ is finite. Now, let us clarify that the case $W_{\kappa_{\beta}+1}^{\beta}\left(q_{i}\right)=\infty$ cannot occur. If we assume that $W_{\kappa_{\beta}+1}^{\beta}\left(q_{i}\right)=\infty$ then we have $q_{i+\kappa_{\beta}+1}=\infty, u\left(i+\kappa_{\beta}\right)=0$ and $j=i+\kappa_{\beta}$. Taking into account that $q_{i}$ is finite, we obtain using Lemma 9 that $q_{i}=0$ and $\beta>2$. Thus, we have that $\kappa_{\beta}=0, i=j$ and that $u(i-1)>u(i)=0=u(j)<u(j+1)$, which is a contradiction.

In the case of a positive semi-wave on $[i-1, j+1]$, i.e. if conditions in (45) hold, we prove statements in an analogous way.

Remark 11. Let $u$ be the solution of (30) for $(\alpha, \beta) \in D$ such that $u(i-1)=0$ and on the interval $[i-2, j+1]$, we have a negative semi-wave (cf. (46) in Lemma 10)

$$
u(i-2)>0, \quad u(i-1)=0, \quad u(k) \leq 0 \quad \text { for } k=i, \ldots, j, \quad u(j+1)>0 .
$$

Then we have $q_{i}=\frac{u(i)}{u(i-1)}=\infty, j=i+\kappa_{\beta}$ and $W_{\kappa_{\beta}+1}^{\beta}\left(q_{i}\right)$ is negative or infinity. Moreover, using Lemma 9, we conclude that $W_{\kappa_{\beta}+1}^{\beta}\left(q_{i}\right)=\infty$ if and only if $\beta=\xi_{k}$, $k \in \mathbb{N}, k \geq 2$.

We provide a new expression for the values of $p_{i}(\alpha, \beta)$ (defined in (32) and (33)) using the Heaviside unit step function $H$ defined as

$$
H(q):= \begin{cases}1 & \text { for } q \geq 0 \\ 0 & \text { for } q<0 \text { or } q=\infty\end{cases}
$$


Lemma 12. For $(\alpha, \beta) \in D$ and $k \in \mathbb{N}$, we have

$$
\begin{aligned}
p_{1}(\alpha, \beta) & =\kappa_{\alpha}+1, \\
p_{2 k}(\alpha, \beta) & =\kappa_{\beta}+1+H\left(W_{\kappa_{\beta}+1}^{\beta}\left(\vartheta_{2 k-1}(\alpha, \beta)\right)\right), \\
p_{2 k+1}(\alpha, \beta) & =\kappa_{\alpha}+1+H\left(W_{\kappa_{\alpha}+1}^{\alpha}\left(\vartheta_{2 k}(\alpha, \beta)\right)\right) .
\end{aligned}
$$

Proof. Firstly, the statement (47) in Lemma 10 can be equivalently written in the following form

$$
j=i+\kappa_{\lambda}+H\left(W_{\kappa_{\lambda}+1}^{\lambda}\left(q_{i}\right)\right) .
$$

Now, let $u$ be the solution of the initial value problem (30) with $C_{1}>0$. Then $u$ has a positive semi-wave on the interval $\left[-1, p_{1}+1\right]$, where $p_{1}$ is defined in (32) as

$$
p_{1}(\alpha, \beta)= \begin{cases}\left\lfloor T^{\alpha}(\infty)+\frac{\pi}{\omega_{\alpha}}\right\rfloor & \text { for } \alpha<4 \\ \left\lfloor T^{\beta}(\infty)+T^{\beta}(2-\alpha)+1\right\rfloor & \text { for } \alpha \geq 4\end{cases}
$$

Since $T^{\alpha}(\infty)=T^{\beta}(\infty)=0$, we have that $p_{1}(\alpha, \beta)=\kappa_{\alpha}+1$. Indeed, for $\alpha \geq 4$, we have $\kappa_{\alpha}=0$ and $0<T^{\beta}(2-\alpha)<\frac{1}{2}$. Moreover, using $(34)$, we have $\vartheta_{1}(\alpha, \beta)=W_{p_{1}(\alpha, \beta)}^{\alpha}(\infty)$ which is exactly the value of $\frac{u\left(p_{1}+1\right)}{u\left(p_{1}\right)}$. The solution $u$ has a negative semi-wave on the interval $\left[p_{1}, p_{1}+p_{2}+1\right]$, where $p_{2}$ is given by (33) in the following way

$$
p_{2}(\alpha, \beta)= \begin{cases}\left\lfloor T^{\beta}\left(\vartheta_{1}(\alpha, \beta)\right)+\frac{\pi}{\omega_{\beta}}\right\rfloor & \text { for } \beta<4 \\ \left\lfloor T^{\alpha}\left(\vartheta_{1}(\alpha, \beta)\right)+T^{\alpha}(2-\beta)+1\right\rfloor & \text { for } \beta \geq 4\end{cases}
$$

Thus, using (48), (54) and (59) for $\lambda=\beta$, we get

$$
p_{2}(\alpha, \beta)=j-i+1=\kappa_{\beta}+H\left(W_{\kappa_{\beta}+1}^{\beta}\left(\vartheta_{1}(\alpha, \beta)\right)\right)+1
$$

which corresponds to (57). Moreover, using (35), we have that $\vartheta_{2}(\alpha, \beta)=$ $W_{p_{2}(\alpha, \beta)}^{\beta}\left(\vartheta_{1}(\alpha, \beta)\right)$ which is equal to $\frac{u\left(p_{1}+p_{2}+1\right)}{u\left(p_{1}+p_{2}\right)}$. And similarly, the solution $u$ has a positive semi-wave on $\left[p_{1}+p_{2}, p_{1}+p_{2}+p_{3}+1\right]$, where $p_{3}$ is defined in (32) as

$$
p_{3}(\alpha, \beta)= \begin{cases}\left\lfloor T^{\alpha}\left(\vartheta_{2}(\alpha, \beta)\right)+\frac{\pi}{\omega_{\alpha}}\right\rfloor & \text { for } \alpha<4, \\ \left\lfloor T^{\beta}\left(\vartheta_{2}(\alpha, \beta)\right)+T^{\beta}(2-\alpha)+1\right\rfloor & \text { for } \alpha \geq 4\end{cases}
$$

Thus, using (59) for $\lambda=\alpha$, we obtain

$$
p_{3}(\alpha, \beta)=j-i+1=\kappa_{\alpha}+H\left(W_{\kappa_{\alpha}+1}^{\alpha}\left(\vartheta_{2}(\alpha, \beta)\right)\right)+1 \text {, }
$$



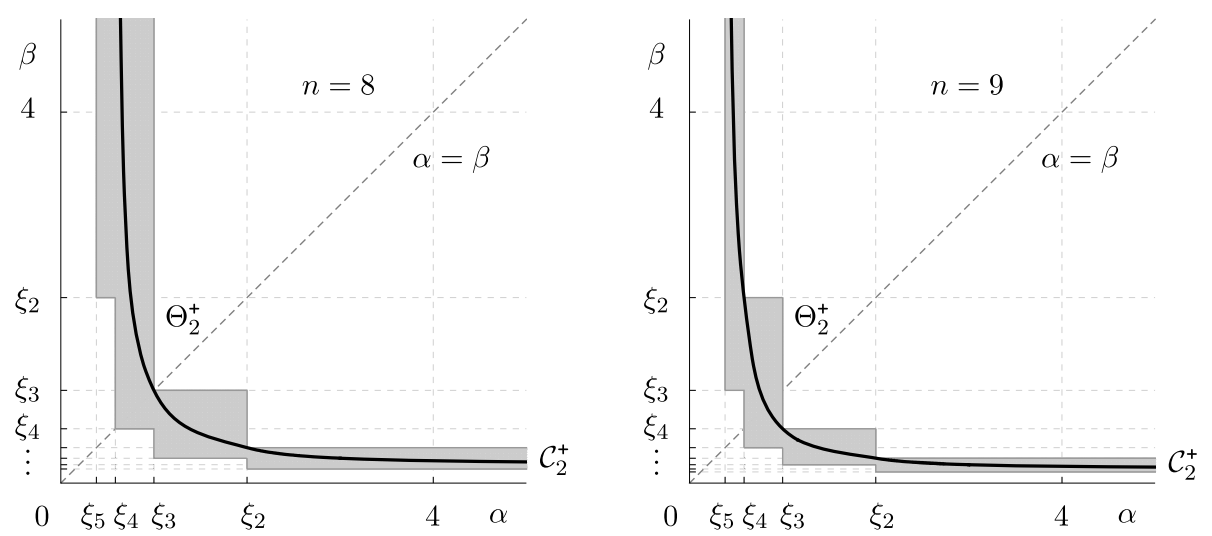

Fig. 22. The set $\Theta_{2}^{+}$(grey region) as the basic bound for the second non-trivial Fučík curve $\mathcal{C}_{2}^{+} \subset \Theta_{2}^{+}$(black curve) for $n=8$ (left) and for $n=9$ (right).
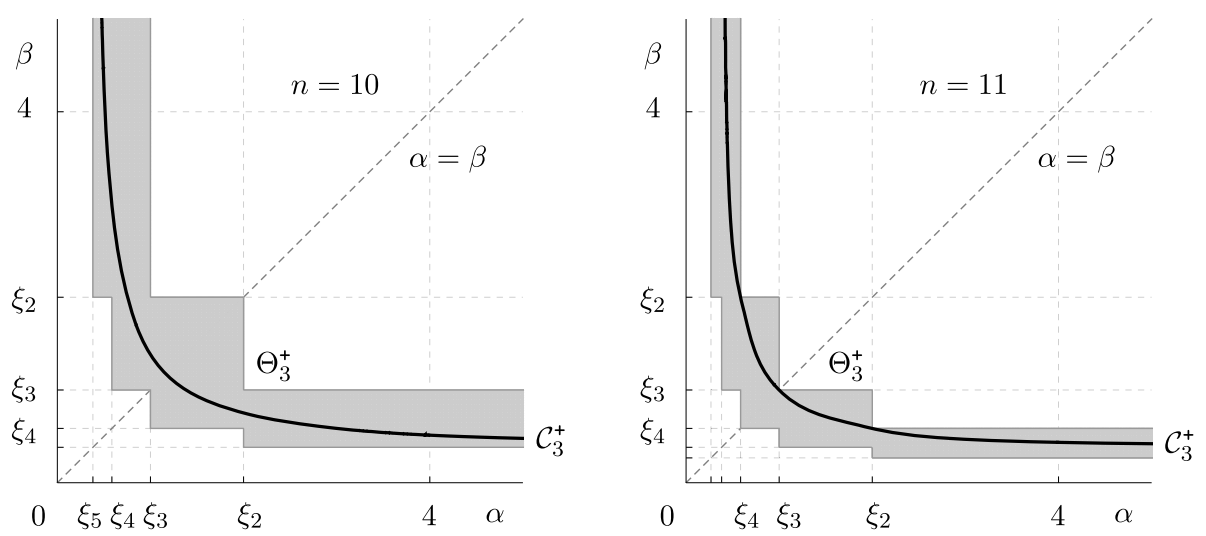

Fig. 23. The set $\Theta_{3}^{+}$(grey region) as the basic bound for the third non-trivial Fučík curve $\mathcal{C}_{3}^{+} \subset \Theta_{3}^{+}$(black curve) for $n=10$ (left) and for $n=11$ (right).

which corresponds to (58). To conclude, we have justified (57) and (58) for $k=1$ (i.e. for $p_{2}$ and $p_{3}$, respectively). In the case of $k \geq 2$, the proof of (57) and (58) concerning $p_{2 k}$ and $p_{2 k+1}$ can be done in an analogous way.

At the end of this section, using Lemma 12, we obtain some basic bounds for each Fučík curve $\mathcal{C}_{l}^{ \pm} \subset \Theta_{l}^{ \pm}$(see Figs. 22 and 23).

Theorem 13. In the domain $D$, we have the following bounds for Fučik curves $\mathcal{C}_{l}^{ \pm} \subset \Theta_{l}^{ \pm}$, $l=1, \ldots, n-1$, where

$$
\begin{gathered}
\Theta_{2 j-1}^{ \pm}:=\left\{(\alpha, \beta) \in D: 0 \leq n+1-j\left(\kappa_{\alpha}+1\right)-j\left(\kappa_{\beta}+1\right) \leq 2 j-1\right\}, \\
\Theta_{2 j}^{+}:=\left\{(\alpha, \beta) \in D: 0 \leq n+1-(j+1)\left(\kappa_{\alpha}+1\right)-j\left(\kappa_{\beta}+1\right) \leq 2 j\right\}, \\
\Theta_{2 j}^{-}:=\left\{(\alpha, \beta) \in D: 0 \leq n+1-j\left(\kappa_{\alpha}+1\right)-(j+1)\left(\kappa_{\beta}+1\right) \leq 2 j\right\} .
\end{gathered}
$$


Proof. First of all, it is enough to focus only on Fučík curves $\mathcal{C}_{l}^{+}$since we have

$$
\mathcal{C}_{l}^{-}=\left\{(\alpha, \beta) \in D:(\beta, \alpha) \in \mathcal{C}_{l}^{+}\right\}
$$

By Theorem 22 in [20], we have that $\mathcal{C}_{l}^{+} \subset \Omega_{l}^{+}$, where $\Omega_{l}^{+}$is the set of all pairs $(\alpha, \beta) \in D$ such that

$$
\sum_{i=1}^{l+1} p_{i}(\alpha, \beta)=n+1 \text {. }
$$

Thus, using Lemma 12, we obtain for $l=2 j-1$ that

$$
j\left(\kappa_{\alpha}+1\right)+j\left(\kappa_{\beta}+1\right) \leq n+1 \leq j\left(\kappa_{\alpha}+1\right)+j\left(\kappa_{\beta}+1\right)+2 j-1
$$

and for $l=2 j$ that

$$
(j+1)\left(\kappa_{\alpha}+1\right)+j\left(\kappa_{\beta}+1\right) \leq n+1 \leq(j+1)\left(\kappa_{\alpha}+1\right)+j\left(\kappa_{\beta}+1\right)+2 j .
$$

\section{Implicit description of Fučík curves}

In this section, we investigate the distance between zeros of two consecutive continuous positive semi-waves of the solution $u$ of the initial value problem (30) for $0<\alpha<4$ and $\beta>0$. Thus, let $i, j \in \mathbb{Z}$ be such that $i<j$ and that (46) holds, i.e. $i$ is the generalized zero of $u$ and the next generalized zero of $u$ is $j$ or $(j+1)$ if $u(j)=0$ or $u(j)<0$, respectively. Moreover, we have two consecutive continuous positive semi-waves $u_{1}^{\mathrm{c}}$ and

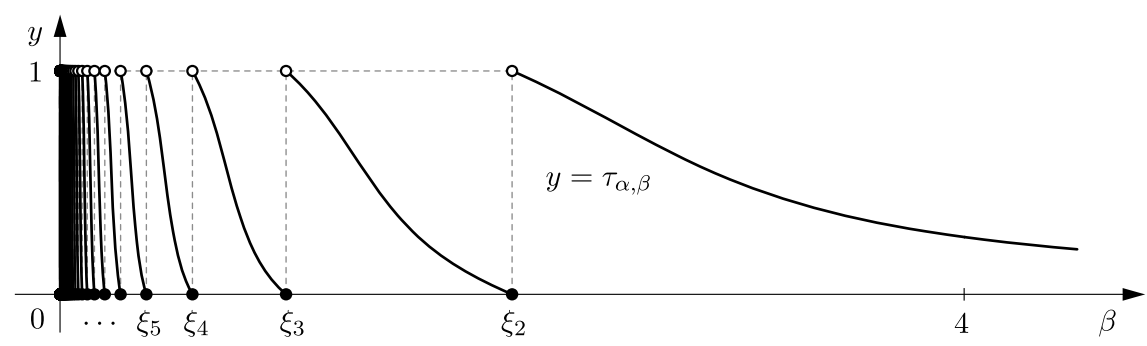

Fig. 24. The graph of the function $\beta \mapsto \tau_{\alpha, \beta}$ for fixed $\alpha=2.9$.

$u_{2}^{\mathrm{c}}$ of $u$ with zeros $t_{1} \in(i-1, i]$ and $t_{2} \in[j, j+1)$, respectively. In the following Lemma 14, we show how to reconstruct the zero $t_{2}$ according to values of $t_{1}, \alpha$ and $\beta$. For this reconstruction, we use $\tau_{\alpha, \beta}=T^{\alpha}\left(V_{\kappa_{\beta}+1}^{\beta} / V_{\kappa_{\beta}}^{\beta}\right)$ introduced in Definition 1 (see Fig. 24) to distinguish between two disjoint cases (see Fig. 25)

$$
j=i+\kappa_{\beta} \quad \text { and } \quad j=i+\kappa_{\beta}+1 \text {. }
$$



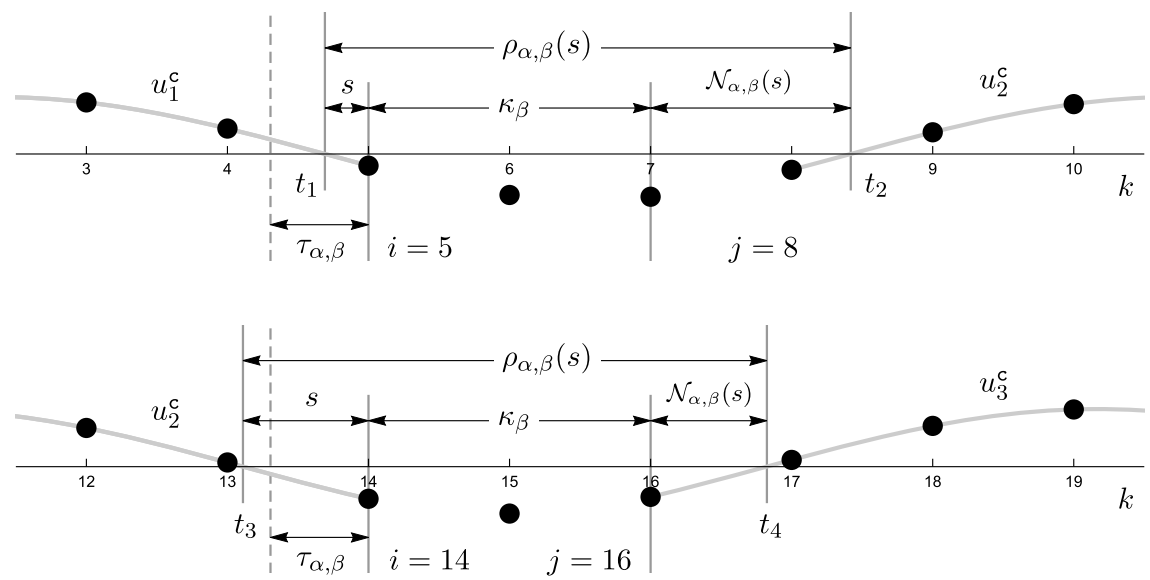

Fig. 25. Two details of the solution $u$ of the initial value problem (30) for fixed $\alpha=0.432$ and $\beta=0.671$ (i.e. $\kappa_{\beta}=2$ ). On top, we have two continuous positive semi-waves $u_{1}^{\mathrm{c}}$ and $u_{2}^{\mathrm{c}}$ with zeros $t_{1}$ and $t_{2}$ such that $j=i+\kappa_{\beta}+1$. Bottom, we have two continuous positive semi-waves $u_{2}^{c}$ and $u_{3}^{c}$ with zeros $t_{3}$ and $t_{4}$ such that $j=i+\kappa_{\beta}$.

Let us note that $0 \leq \tau_{\alpha, \beta}<1$ since $V_{\kappa_{\beta}+1}^{\beta} / V_{\kappa_{\beta}}^{\beta}$ is negative or equal to $\infty$ according to Lemma 7.

Lemma 14. Let $u$ be the solution of the initial value problem (30) for $0<\alpha<4$ and $\beta>0$ and let $u_{1}^{\mathrm{c}}$ and $u_{2}^{\mathrm{c}}$ be two consecutive continuous positive semi-waves of $u$. Moreover, let $t_{1}$ be the second zero of $u_{1}^{\mathrm{c}}$ and let $t_{2}$ be the first zero of $u_{2}^{\mathrm{c}}$. If we denote $s=\left\lceil t_{1}\right\rceil-t_{1}$ then we have

$$
t_{2}= \begin{cases}t_{1}+s+\kappa_{\beta}+T^{\alpha}\left(W_{\kappa_{\beta}+1}^{\beta}\left(Q^{\alpha}(1-s)\right)\right) & \text { for } s>\tau_{\alpha, \beta}, \\ t_{1}+s+\kappa_{\beta}+1+T^{\alpha}\left(W_{\kappa_{\beta}+2}^{\beta}\left(Q^{\alpha}(1-s)\right)\right) & \text { for } s \leq \tau_{\alpha, \beta} .\end{cases}
$$

Proof. We have $t_{1} \in(i-1, i]$ and $t_{2} \in[j, j+1)$, where $i, j \in \mathbb{Z}$ are such that $i<j$ and that (46) holds. Moreover, we have

$$
q_{i}=Q^{\alpha}(1-s), \quad q_{j+1}=W_{j-i+1}^{\beta}\left(q_{i}\right),
$$

where we denoted $q_{k}:=\frac{u(k)}{u(k-1)}$ for $k=i, \ldots, j+1$. Now, using Lemma 10 and (47) for $\lambda=\beta$, we obtain

$$
j= \begin{cases}i+\kappa_{\beta} & \text { for } W_{\kappa_{\beta}+1}^{\beta}\left(q_{i}\right)<0 \\ i+\kappa_{\beta}+1 & \text { for } W_{\kappa_{\beta}+1}^{\beta}\left(q_{i}\right) \geq 0\end{cases}
$$

Since $t_{2}-j=t_{2}-\left\lfloor t_{2}\right\rfloor=T^{\alpha}\left(q_{j+1}\right)$, we get using the second equality in (61) that $t_{2}=j+T^{\alpha}\left(W_{j-i+1}^{\beta}\left(q_{i}\right)\right)$, which implies using (62) that 


$$
t_{2}= \begin{cases}i+\kappa_{\beta}+T^{\alpha}\left(W_{\kappa_{\beta}+1}^{\beta}\left(q_{i}\right)\right) & \text { for } W_{\kappa_{\beta}+1}^{\beta}\left(q_{i}\right)<0, \\ i+\kappa_{\beta}+1+T^{\alpha}\left(W_{\kappa_{\beta}+2}^{\beta}\left(q_{i}\right)\right) & \text { for } W_{\kappa_{\beta}+1}^{\beta}\left(q_{i}\right) \geq 0 .\end{cases}
$$

Using the first equality in (61), the inequality $W_{\kappa_{\beta}+1}^{\beta}\left(q_{i}\right)<0$ in $(63)$ reads $Q^{\alpha}(1-s)<$ $V_{\kappa_{\beta}}^{\beta} / V_{\kappa_{\beta}+1}^{\beta}$ which can be equivalently written as $Q^{\alpha}(s)>V_{\kappa_{\beta}+1}^{\beta} / V_{\kappa_{\beta}}^{\beta}$ or as $s>\tau_{\alpha, \beta}$ due to (29). Similarly, we obtain that the second inequality $W_{\kappa_{\beta}+1}^{\beta}\left(q_{i}\right) \geq 0$ in (63) reads $s \leq \tau_{\alpha, \beta}$. To conclude, (63) can be also written in the following way

$$
t_{2}= \begin{cases}i+\kappa_{\beta}+T^{\alpha}\left(W_{\kappa_{\beta}+1}^{\beta}\left(q_{i}\right)\right) & \text { for } s>\tau_{\alpha, \beta}, \\ i+\kappa_{\beta}+1+T^{\alpha}\left(W_{\kappa_{\beta}+2}^{\beta}\left(q_{i}\right)\right) & \text { for } s \leq \tau_{\alpha, \beta},\end{cases}
$$

which is exactly (60) if we take into account the first equality in (61) and that $i=\left\lceil t_{1}\right\rceil=$ $t_{1}+s$.

Remark 15. Let us note that for $s=\tau_{\alpha, \beta}$, we have using (60) that $t_{2}=t_{1}+s+\kappa_{\beta}+1$, since $Q^{\alpha}\left(1-\tau_{\alpha, \beta}\right)=1 / Q^{\alpha}\left(\tau_{\alpha, \beta}\right)=V_{\kappa_{\beta}}^{\beta} / V_{\kappa_{\beta}+1}^{\beta}$ and thus

$$
T^{\alpha}\left(W_{\kappa_{\beta}+2}^{\beta}\left(Q^{\alpha}\left(1-\tau_{\alpha, \beta}\right)\right)\right)=T^{\alpha}(\infty)=0
$$

See also Fig. 20 and note that for $q=V_{\kappa_{\beta}}^{\beta} / V_{\kappa_{\beta}+1}^{\beta}$, we have $W_{\kappa_{\beta}+1}^{\beta}(q)=0$ and $W_{\kappa_{\beta}+2}^{\beta}(q)=$ $W_{1}^{\beta}\left(W_{\kappa_{\beta}+1}^{\beta}(q)\right)=\infty$.

In the following lemma, we provide some basic properties of $\tau_{\alpha, \beta}$ (see Fig. 24).

Lemma 16. For $0<\alpha<4$ and $\beta>0$, we have that $0 \leq \tau_{\alpha, \beta}<1$. Moreover, if we denote

$$
\xi_{k}:=4 \sin ^{2} \frac{\pi}{2 k}, \quad \zeta_{k}:=4 \sin ^{2} \frac{\pi}{2 k-1} \quad \text { for } k \in \mathbb{N}, k \geq 2
$$

then we have

1. $\tau_{\alpha, \beta}=0$ if and only if $\beta=\xi_{k}$ for some $k \in \mathbb{N}, k \geq 2$,

2. $\tau_{\alpha, \beta}=\frac{1}{2}$ if and only if $\beta=\zeta_{k}$ for some $k \in \mathbb{N}, k \geq 2$,

3. $\tau_{\beta, \beta}=\frac{\pi}{\omega_{\beta}}-\left\lfloor\frac{\pi}{\omega_{\beta}}\right\rfloor$ for $0<\beta<4$.

Proof. First of all, for $0<\alpha<4$ and $\beta \geq 4$, we have that $\tau_{\alpha, \beta}=T^{\alpha}\left(\frac{V_{1}^{\beta}}{V_{0}^{\beta}}\right)=T^{\alpha}(2-\beta)$ and thus, $0<\tau_{\alpha, \beta}<\frac{1}{2}$ since $(2-\beta)<-1$. For the rest of the proof, let us restrict to the case $0<\beta<4$. We claim that

$$
\tau_{\alpha, \beta}=T^{\alpha}\left(Q^{\beta}\left(\frac{\pi}{\omega_{\beta}}-\left\lfloor\frac{\pi}{\omega_{\beta}}\right\rfloor\right)\right) \quad \text { for } 0<\alpha, \beta<4
$$


Indeed, using Lemma 8, we get

$$
Q^{\beta}\left(\frac{\pi}{\omega_{\beta}}-\left\lfloor\frac{\pi}{\omega_{\beta}}\right\rfloor\right)=W_{\kappa_{\beta}+1}^{\beta}(\infty)=\frac{V_{\kappa_{\beta}+1}^{\beta}}{V_{\kappa_{\beta}}^{\beta}},
$$

which justifies (64) according to the definition of $\tau_{\alpha, \beta}$. Now, since $0<\frac{\pi}{\omega_{\beta}}-\left\lfloor\frac{\pi}{\omega_{\beta}}\right\rfloor<1$ or $\frac{\pi}{\omega_{\beta}}-\left\lfloor\frac{\pi}{\omega_{\beta}}\right\rfloor=0$, we have that

$$
Q^{\beta}\left(\frac{\pi}{\omega_{\beta}}-\left\lfloor\frac{\pi}{\omega_{\beta}}\right\rfloor\right)<0 \quad \text { or } \quad Q^{\beta}\left(\frac{\pi}{\omega_{\beta}}-\left\lfloor\frac{\pi}{\omega_{\beta}}\right\rfloor\right)=\infty
$$

respectively. Thus, using (64), we get that $0<\tau_{\alpha, \beta}<1$ for $\left\lfloor\frac{\pi}{\omega_{\beta}}\right\rfloor<\frac{\pi}{\omega_{\beta}}$ and that $\tau_{\alpha, \beta}=0$ for $\left\lfloor\frac{\pi}{\omega_{\beta}}\right\rfloor=\frac{\pi}{\omega_{\beta}}$. Moreover, $\left\lfloor\frac{\pi}{\omega_{\beta}}\right\rfloor=\frac{\pi}{\omega_{\beta}}$ if and only if $\frac{\pi}{\omega_{\beta}}=k, k \in \mathbb{N}, k \geq 2$ (recall that $\left.1<\frac{\pi}{\omega_{\beta}}\right)$, and let us note that $\frac{\pi}{\omega_{\beta}}=k$ reads $\beta=2-2 \cos \frac{\pi}{k}$ or $\beta=\xi_{k}$. Finally, using (64), we conclude that $\tau_{\alpha, \beta}=\frac{1}{2}$ can be equivalently written as $Q^{\beta}\left(\frac{\pi}{\omega_{\beta}}-\left\lfloor\frac{\pi}{\omega_{\beta}}\right\rfloor\right)=-1$ or $\frac{\pi}{\omega_{\beta}}=\left\lfloor\frac{\pi}{\omega_{\beta}}\right\rfloor+\frac{1}{2}$ or $\frac{\pi}{\omega_{\beta}}=k+\frac{1}{2}, k \in \mathbb{N}, k \geq 2$, and let us note that $\frac{\pi}{\omega_{\beta}}=k+\frac{1}{2}$ reads $\beta=2-2 \cos \frac{2 \pi}{2 k+1}$ or $\beta=\zeta_{k}$.

Let us introduce the function $\mathcal{N}_{\alpha, \beta}:\left[0,1+\tau_{\alpha, \beta}\right] \rightarrow \mathbb{R}$, which we use to measure the distance $t_{2}-t_{1}$ between zeros $t_{1}$ and $t_{2}$ of two consecutive continuous positive semi-waves of $u$ (see Figs. 25 and 26).

Definition 17. For $0<\alpha<4$ and $\beta>0$, let us define

$$
\operatorname{Dom}\left(\mathcal{N}_{\alpha, \beta}\right):=\left[0,1+\tau_{\alpha, \beta}\right], \quad \mathcal{N}_{\alpha, \beta}(s):= \begin{cases}\overline{\bar{M}}_{\alpha, \beta}(s)+1 & \text { for } s \in\left[0, \tau_{\alpha, \beta}\right], \\ \bar{M}_{\alpha, \beta}(s) & \text { for } s \in\left(\tau_{\alpha, \beta}, 1\right), \\ \bar{M}_{\alpha, \beta}(s-1) & \text { for } s \in\left[1,1+\tau_{\alpha, \beta}\right]\end{cases}
$$

where

$$
\begin{array}{lll}
\bar{M}_{\alpha, \beta}(s):=T^{\alpha}\left(W_{\kappa_{\beta}+1}^{\beta}\left(Q^{\alpha}(1-s)\right)\right), & s \in\left[\tau_{\alpha, \beta}, 1\right], \\
\bar{M}_{\alpha, \beta}(s):=T^{\alpha}\left(W_{\kappa_{\beta}+2}^{\beta}\left(Q^{\alpha}(1-s)\right)\right), & s \in\left[0, \tau_{\alpha, \beta}\right] .
\end{array}
$$

Remark 18. Let $u$ be the solution of the initial value problem (30) for $0<\alpha<4$ and $\beta>0$ and let $t_{1}$ and $t_{2}$ be two zeros of positive semi-waves of $u$ as in Lemma 14 (see Fig. 25). Then we have

$$
t_{2}=t_{1}+s+\kappa_{\beta}+\mathcal{N}_{\alpha, \beta}(s), \quad 0 \leq s \leq 1+\tau_{\alpha, \beta} .
$$

Indeed, for $0 \leq s<1$, we have that $s=\left\lceil t_{1}\right\rceil-t_{1}$ and we obtain using (60) that 


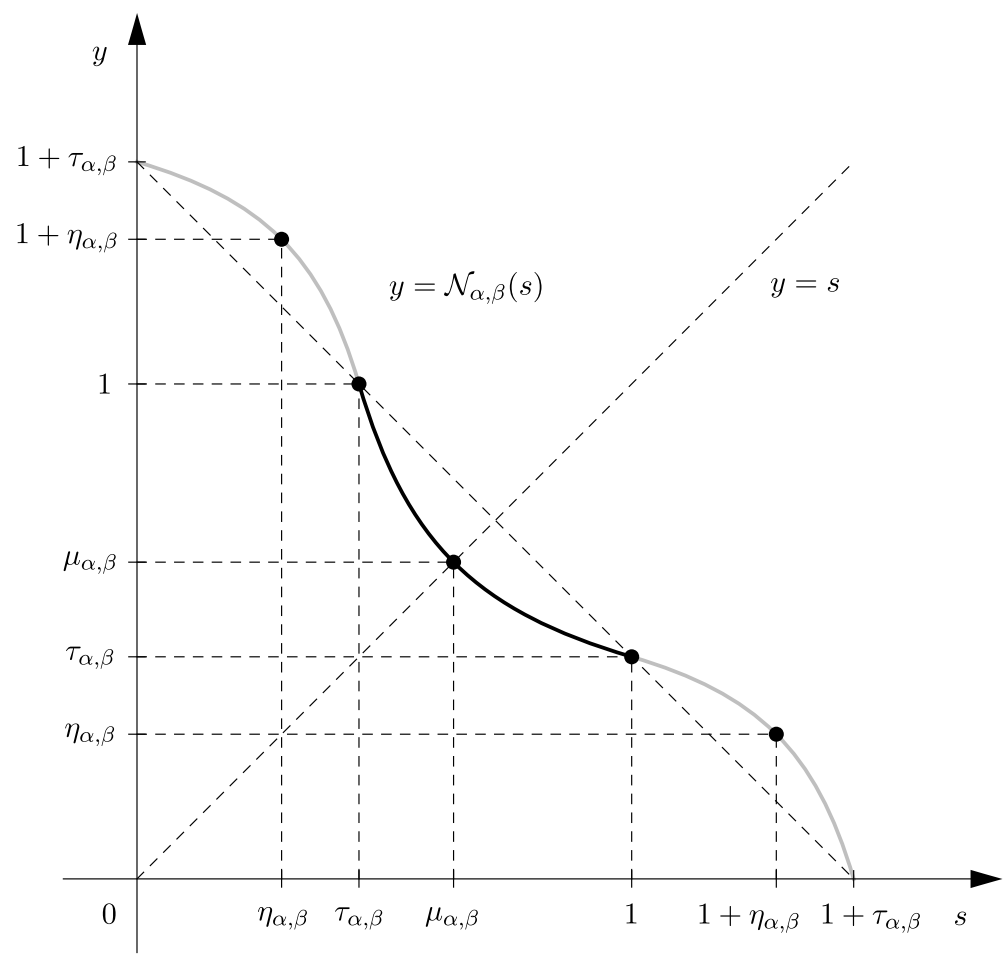

Fig. 26. The graph of the function $\mathcal{N}_{\alpha, \beta}=\mathcal{N}_{\alpha, \beta}(s)$ for fixed $\alpha=1.2$ and $\beta=3.2$.

$$
t_{2}= \begin{cases}t_{1}+s+\kappa_{\beta}+1+\overline{\bar{M}}_{\alpha, \beta}(s) & \text { for } 0 \leq s \leq \tau_{\alpha, \beta} \\ t_{1}+s+\kappa_{\beta}+\bar{M}_{\alpha, \beta}(s) & \text { for } \tau_{\alpha, \beta}<s<1\end{cases}
$$

Moreover, for $1 \leq s \leq 1+\tau_{\alpha, \beta}$, we have $0 \leq s-1 \leq \tau_{\alpha, \beta}$ and thus,

$$
t_{2}=t_{1}+s-1+\kappa_{\beta}+1+\overline{\bar{M}}_{\alpha, \beta}(s-1)=t_{1}+s+\kappa_{\beta}+\mathcal{N}_{\alpha, \beta}(s) .
$$

Let us introduce the function $\rho_{\alpha, \beta}$ according to (65), which measures the distance between zeros of two consecutive continuous positive semi-waves.

Definition 19. Let $0<\alpha<4$ and $\beta>0$. Let us define

$$
\rho_{\alpha, \beta}(s):=s+\kappa_{\beta}+\mathcal{N}_{\alpha, \beta}(s), \quad 0 \leq s \leq 1+\tau_{\alpha, \beta} .
$$

Now, using (65), we have for zeros $t_{1}$ and $t_{2}$ in Lemma 14 that

$$
t_{2}=t_{1}+\rho_{\alpha, \beta}\left(\left\lceil t_{1}\right\rceil-t_{1}\right)
$$

In the following three lemmas, let us investigate some basic properties of $\mathcal{N}_{\alpha, \beta}$. 


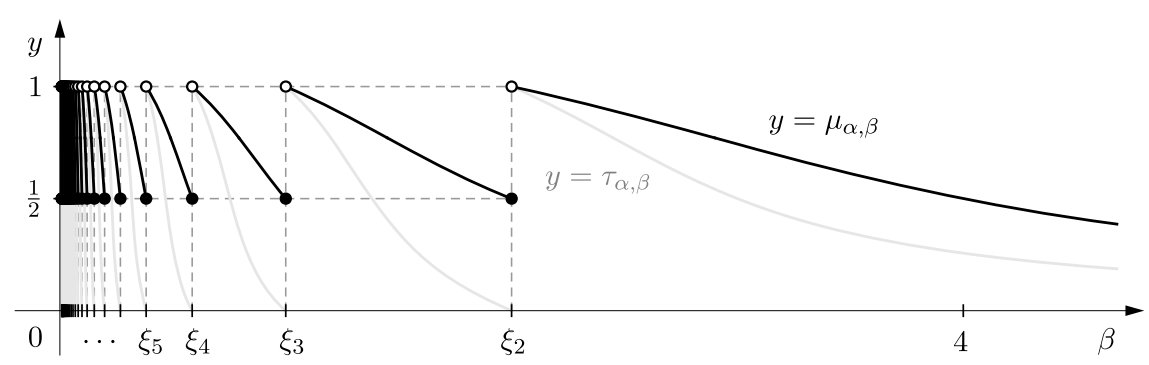

Fig. 27. The graph of the function $\beta \mapsto \mu_{\alpha, \beta}$ for fixed $\alpha=2.9$.

Lemma 20. The function $\mathcal{N}_{\alpha, \beta}$ is a continuous involution, i.e.

$$
\forall s \in\left[0,1+\tau_{\alpha, \beta}\right]: \mathcal{N}_{\alpha, \beta}\left(\mathcal{N}_{\alpha, \beta}(s)\right)=s .
$$

Moreover, we have $\mathcal{N}_{\alpha, \beta}(0)=1+\tau_{\alpha, \beta}$ and $\mathcal{N}_{\alpha, \beta}\left(\tau_{\alpha, \beta}\right)=1$.

Proof. At first, $\bar{M}_{\alpha, \beta}$ is the continuous strictly decreasing function on $\left[\tau_{\alpha, \beta}, 1\right]$, which maps this interval onto itself. Moreover, $\bar{M}_{\alpha, \beta}$ is an involution. Indeed, for $\tau_{\alpha, \beta} \leq s \leq 1$, we have

$$
\bar{M}_{\alpha, \beta}(s)=T^{\alpha}\left(W_{\kappa_{\beta}+1}^{\beta}\left(1 / Q^{\alpha}(s)\right)\right)=T^{\alpha}\left(1 / W_{-\kappa_{\beta}-1}^{\beta}\left(Q^{\alpha}(s)\right)\right)
$$

and thus, we obtain

$$
\bar{M}_{\alpha, \beta}\left(\bar{M}_{\alpha, \beta}(s)\right)=T^{\alpha}\left(1 / W_{-\kappa_{\beta}-1}^{\beta}\left(Q^{\alpha}\left(T^{\alpha}\left(W_{\kappa_{\beta}+1}^{\beta}\left(1 / Q^{\alpha}(s)\right)\right)\right)\right)\right)=s .
$$

At second, $\overline{\bar{M}}_{\alpha, \beta}$ is the continuous strictly decreasing function on $\left[0, \tau_{\alpha, \beta}\right]$, which maps this interval onto itself. Moreover, $\overline{\bar{M}}_{\alpha, \beta}$ is an involution, which can be justified similarly as in the case of $\bar{M}_{\alpha, \beta}$.

Finally, $\mathcal{N}_{\alpha, \beta}$ is the continuous strictly decreasing function on $\left[0,1+\tau_{\alpha, \beta}\right]$, which maps this interval onto itself, and it is an involution. Indeed, for $0 \leq s \leq \tau_{\alpha, \beta}$, we have

$$
\mathcal{N}_{\alpha, \beta}\left(\mathcal{N}_{\alpha, \beta}(s)\right)=\overline{\bar{M}}_{\alpha, \beta}\left(\overline{\bar{M}}_{\alpha, \beta}(s)+1-1\right)=s
$$

and for $1 \leq s \leq 1+\tau_{\alpha, \beta}$, we have

$$
\mathcal{N}_{\alpha, \beta}\left(\mathcal{N}_{\alpha, \beta}(s)\right)=\overline{\bar{M}}_{\alpha, \beta}\left(\overline{\bar{M}}_{\alpha, \beta}(s-1)\right)+1=s-1+1=s
$$

Now, let us focus on $\eta_{\alpha, \beta}$ and $\mu_{\alpha, \beta}$ introduced in Definition 1 (see Figs. 27 and 28). Using Lemma 7, we get that $0 \leq \eta_{\alpha, \beta}<1$ and $0<\mu_{\alpha, \beta}<1$ since $\left(V_{\kappa_{\beta}+1}^{\beta}-1\right) / V_{\kappa_{\beta}}^{\beta}$ is negative or equal to $\infty$ and $V_{\kappa_{\beta}+1}^{\beta} /\left(V_{\kappa_{\beta}}^{\beta}+1\right)$ is negative. 


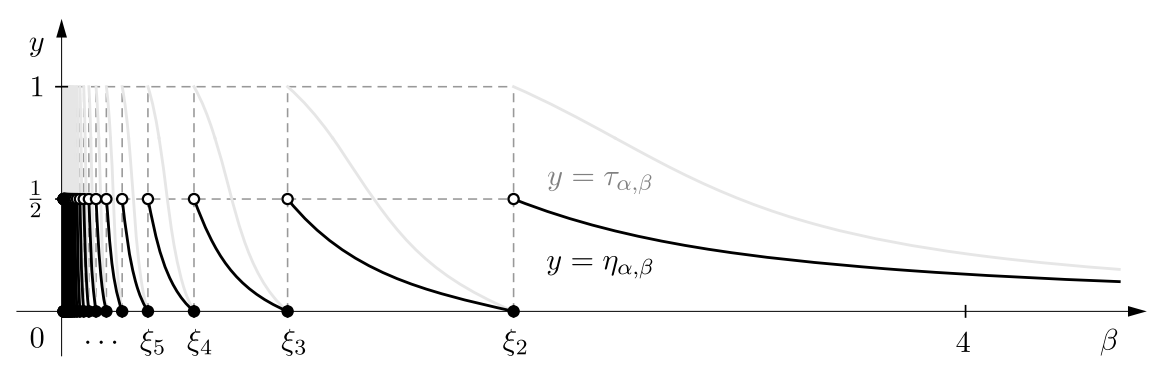

Fig. 28. The graph of the function $\beta \mapsto \eta_{\alpha, \beta}$ for fixed $\alpha=2.9$.

Lemma 21. The points $\eta_{\alpha, \beta}$ and $\mu_{\alpha, \beta}$ are fixed points of $\overline{\bar{M}}_{\alpha, \beta}$ and $\bar{M}_{\alpha, \beta}$, respectively. Moreover, we have

$$
\mathcal{N}_{\alpha, \beta}\left(\eta_{\alpha, \beta}\right)=\eta_{\alpha, \beta}+1, \quad \mathcal{N}_{\alpha, \beta}\left(\mu_{\alpha, \beta}\right)=\mu_{\alpha, \beta}
$$

Proof. Using (29), we obtain

$$
\overline{\bar{M}}_{\alpha, \beta}\left(\eta_{\alpha, \beta}\right)=T^{\alpha}\left(W_{\kappa_{\beta}+2}^{\beta}\left(\frac{V_{\kappa_{\beta}}^{\beta}}{V_{\kappa_{\beta}+1}^{\beta}-1}\right)\right)=T^{\alpha}\left(\frac{V_{\kappa_{\beta}+1}^{\beta}-1}{V_{\kappa_{\beta}}^{\beta}}\right)=\eta_{\alpha, \beta},
$$

where we used (40) to simplify

$$
W_{\kappa_{\beta}+2}^{\beta}\left(\frac{V_{\kappa_{\beta}}^{\beta}}{V_{\kappa_{\beta}+1}^{\beta}-1}\right)=\frac{V_{\kappa_{\beta}+1}^{\beta}-\left(V_{\kappa_{\beta}+1}^{\beta} V_{\kappa_{\beta}+1}^{\beta}-V_{\kappa_{\beta}}^{\beta} V_{\kappa_{\beta}+2}^{\beta}\right)}{V_{\kappa_{\beta}}^{\beta}}=\frac{V_{\kappa_{\beta}+1}^{\beta}-1}{V_{\kappa_{\beta}}^{\beta}} .
$$

In a similar way, we show that $\bar{M}_{\alpha, \beta}\left(\mu_{\alpha, \beta}\right)=\mu_{\alpha, \beta}$. Finally, we have $\mathcal{N}_{\alpha, \beta}\left(\eta_{\alpha, \beta}\right)=$ $\overline{\bar{M}}_{\alpha, \beta}\left(\eta_{\alpha, \beta}\right)+1=\eta_{\alpha, \beta}+1$ and $\mathcal{N}_{\alpha, \beta}\left(\mu_{\alpha, \beta}\right)=\bar{M}_{\alpha, \beta}\left(\mu_{\alpha, \beta}\right)=\mu_{\alpha, \beta}$, which finishes the proof.

Lemma 22. Let $0<\alpha<4$.

1. If $\beta=\xi_{k}, k \in \mathbb{N}, k \geq 2$, then $\mathcal{N}_{\alpha, \beta}(s)=1-s$.

2. If $\beta=\alpha$ then $\mathcal{N}_{\alpha, \beta}(s)=1-s+\tau_{\beta, \beta}=1-s+\frac{\pi}{\omega_{\beta}}-\left\lfloor\frac{\pi}{\omega_{\beta}}\right\rfloor$.

Proof. At first, let us assume that $\beta=\xi_{k}, k \in \mathbb{N}, k \geq 2$. Using Lemma 16, we obtain that $\tau_{\alpha, \beta}=0$ and thus, we have that $\mathcal{N}_{\alpha, \beta}(0)=1$ and $\mathcal{N}_{\alpha, \beta}(1)=0$. Moreover, since $W_{\kappa_{\beta}+1}^{\beta}$ is the identity function (recall (42)), we have for $0<s<1$ that

$$
\mathcal{N}_{\alpha, \beta}(s)=\bar{M}_{\alpha, \beta}(s)=T^{\alpha}\left(W_{\kappa_{\beta}+1}^{\beta}\left(Q^{\alpha}(1-s)\right)\right)=1-s .
$$


At second, let us assume that $0<\beta=\alpha<4$. Then using (64), we obtain

$$
\frac{\pi}{\omega_{\beta}}=t_{2}-t_{1}=s+\kappa_{\beta}+\mathcal{N}_{\alpha, \beta}(s), \quad 0 \leq s \leq 1+\tau_{\beta, \beta}
$$

where $\tau_{\beta, \beta}=\frac{\pi}{\omega_{\beta}}-\left\lfloor\frac{\pi}{\omega_{\beta}}\right\rfloor$ due to Lemma 16. Finally, using (67), we get

$$
\mathcal{N}_{\alpha, \beta}(s)=\frac{\pi}{\omega_{\beta}}-s-\kappa_{\beta}=\frac{\pi}{\omega_{\beta}}-s-\left\lfloor\frac{\pi}{\omega_{\beta}}\right\rfloor+1=1-s+\tau_{\beta, \beta}
$$

In the following lemma, we show that values $\eta_{\alpha, \beta}, \tau_{\alpha, \beta}$ and $\mu_{\alpha, \beta}$ are always ordered in one way (see Figs. 27 and 28).

Lemma 23. For $0<\alpha<4$ and $\beta>0$, we have that

$$
0<\eta_{\alpha, \beta}<\tau_{\alpha, \beta}<\mu_{\alpha, \beta}<1 \quad \text { if } \beta \neq \xi_{k}, k \in \mathbb{N}, k \geq 2
$$

Moreover, we have

1. $\eta_{\alpha, \beta}=0$ if and only if $\beta=\xi_{k}$ for some $k \in \mathbb{N}, k \geq 2$,

2. $\mu_{\alpha, \beta}=\frac{1}{2}$ if and only if $\beta=\xi_{k}$ for some $k \in \mathbb{N}$.

Proof. At first, for $\beta \neq \xi_{k}, k \in \mathbb{N}, k \geq 2$, we have that

$$
\frac{V_{\kappa_{\beta}+1}^{\beta}-1}{V_{\kappa_{\beta}}^{\beta}}<\frac{V_{\kappa_{\beta}+1}^{\beta}}{V_{\kappa_{\beta}}^{\beta}}<\frac{V_{\kappa_{\beta}+1}^{\beta}}{V_{\kappa_{\beta}}^{\beta}+1}<0
$$

which implies $0<\eta_{\alpha, \beta}<\tau_{\alpha, \beta}<\mu_{\alpha, \beta}<1$. At second, for $\beta=\xi_{k}, k \in \mathbb{N}, k \geq 2$, we have $V_{\kappa_{\beta}}^{\beta}=0, V_{\kappa_{\beta}+1}^{\beta}=-1$, and thus, $\eta_{\alpha, \beta}=\tau_{\alpha, \beta}=T^{\alpha}(\infty)=0$ and $\mu_{\alpha, \beta}=T^{\alpha}(-1)=$ $\frac{1}{2}$. At third, for $\beta=4$, we have $\kappa_{\beta}=0, V_{\kappa_{\beta}}^{\beta}=1, V_{\kappa_{\beta}+1}^{\beta}=2-\beta=-2$ and thus, $\mu_{\alpha, \beta}=T^{\alpha}(-1)=\frac{1}{2}$. Finally, let us assume that $\beta \neq 4$ and $\mu_{\alpha, \beta}=\frac{1}{2}$. This means that $\frac{V_{\kappa_{\beta}+1}^{\beta}}{V_{\kappa_{\beta}}^{\beta}+1}=-1$ and thus, we have

$$
V_{\kappa_{\beta}}^{\beta}+V_{\kappa_{\beta}+1}^{\beta}=-1
$$

Thus, using Lemma 7 , we obtain that $\beta=\xi_{k}$ for some $k \in \mathbb{N}, k \geq 2$.

Let us reveal a close connection among values $\eta_{\alpha, \beta}, \tau_{\alpha, \beta}$ and $\mu_{\alpha, \beta}$ using the function $G^{\beta}: \mathbb{R} \rightarrow \mathbb{R}^{*}$ defined in the following way

$$
G^{\beta}(q):=\frac{2 q-(2-\beta) q^{2}}{1-q^{2}} \text { for } q \neq \pm 1, \quad G^{\beta}( \pm 1):=\infty
$$


Lemma 24. For $0<\alpha, \beta<4$, we have

$$
\begin{aligned}
\tau_{\alpha, \beta} & =1-T^{\alpha}\left(G^{\beta}\left(Q^{\alpha}\left(1-\eta_{\alpha, \beta}\right)\right)\right) \\
\tau_{\alpha, \beta} & =T^{\alpha}\left(G^{\beta}\left(Q^{\alpha}\left(\mu_{\alpha, \beta}\right)\right)\right) \\
G^{\beta}\left(Q^{\alpha}\left(1-\eta_{\alpha, \beta}\right)\right) & =\frac{1}{G^{\beta}\left(Q^{\alpha}\left(\mu_{\alpha, \beta}\right)\right)} .
\end{aligned}
$$

Proof. Firstly, let us assume that $\beta=\xi_{k}$ for some $k \in \mathbb{N}, k \geq 2$. According to Lemmas 16 and 23, we have $\eta_{\alpha, \beta}=\tau_{\alpha, \beta}=0$ and $\mu_{\alpha, \beta}=\frac{1}{2}$. Moreover, we have

$$
\begin{aligned}
1-T^{\alpha}\left(G^{\beta}\left(Q^{\alpha}\left(1-\eta_{\alpha, \beta}\right)\right)\right) & =1-T^{\alpha}\left(G^{\beta}\left(Q^{\alpha}(1)\right)\right)=1-T^{\alpha}\left(G^{\beta}(0)\right)=1-T^{\alpha}(0) \\
& =0=\tau_{\alpha, \beta}
\end{aligned}
$$

and

$$
T^{\alpha}\left(G^{\beta}\left(Q^{\alpha}\left(\mu_{\alpha, \beta}\right)\right)\right)=T^{\alpha}\left(G^{\beta}\left(Q^{\alpha}\left(\frac{1}{2}\right)\right)\right)=T^{\alpha}\left(G^{\beta}(-1)\right)=T^{\alpha}(\infty)=0=\tau_{\alpha, \beta} .
$$

Secondly, let us assume that $\beta \neq \xi_{k}, k \in \mathbb{N}, k \geq 2$. We claim that $\eta_{\alpha, \beta} \neq \frac{1}{2}$. Indeed, if we assume that $\eta_{\alpha, \beta}=\frac{1}{2}$ then we get $\left(V_{\kappa_{\beta}+1}^{\beta}-1\right) / V_{\kappa_{\beta}}^{\beta}=-1$ and

$$
V_{\kappa_{\beta}}^{\beta}+V_{\kappa_{\beta}+1}^{\beta}=1
$$

which is a contradiction since $V_{\kappa_{\beta}}^{\beta}+V_{\kappa_{\beta}+1}^{\beta}<1$ according to Lemma 7 . Thus, $0<\eta_{\alpha, \beta}<1$, $0<1-\eta_{\alpha, \beta}<1, Q^{\alpha}\left(1-\eta_{\alpha, \beta}\right)<0$ and

$$
Q^{\alpha}\left(1-\eta_{\alpha, \beta}\right) \neq-1, \quad Q^{\alpha}\left(\eta_{\alpha, \beta}\right) \neq-1
$$

Now, using (29), we have

$$
G^{\beta}\left(Q^{\alpha}\left(1-\eta_{\alpha, \beta}\right)\right)=G^{\beta}\left(\frac{1}{Q^{\alpha}\left(\eta_{\alpha, \beta}\right)}\right)=\frac{2 Q^{\alpha}\left(\eta_{\alpha, \beta}\right)-(2-\beta)}{\left(Q^{\alpha}\left(\eta_{\alpha, \beta}\right)\right)^{2}-1}
$$

and thus, using (39), we obtain

$$
\begin{aligned}
G^{\beta}\left(Q^{\alpha}\left(1-\eta_{\alpha, \beta}\right)\right) & =V_{\kappa_{\beta}}^{\beta} \frac{2\left(V_{\kappa_{\beta}+1}^{\beta}-1\right)-(2-\beta) V_{\kappa_{\beta}}^{\beta}}{\left(V_{\kappa_{\beta}+1}^{\beta}-1\right)^{2}-\left(V_{\kappa_{\beta}}^{\beta}\right)^{2}} \\
& =V_{\kappa_{\beta}}^{\beta} \frac{2\left(V_{\kappa_{\beta}+1}^{\beta}-1\right)-\left(V_{\kappa_{\beta}+1}^{\beta}+V_{\kappa_{\beta}-1}^{\beta}\right)}{\left(V_{\kappa_{\beta}+1}^{\beta}-1\right)^{2}-\left(1+V_{\kappa_{\beta}+1}^{\beta} V_{\kappa_{\beta}-1}^{\beta}\right)} \\
& =V_{\kappa_{\beta}}^{\beta} \frac{V_{\kappa_{\beta}+1}^{\beta}-2-V_{\kappa_{\beta}-1}^{\beta}}{\left(V_{\kappa_{\beta}+1}^{\beta}\right)^{2}-2 V_{\kappa_{\beta}+1}^{\beta}-V_{\kappa_{\beta}+1}^{\beta} V_{\kappa_{\beta}-1}^{\beta}}=\frac{V_{\kappa_{\beta}}^{\beta}}{V_{\kappa_{\beta}+1}^{\beta}} .
\end{aligned}
$$


Using (27) and (72), we get

$$
1-T^{\alpha}\left(G^{\beta}\left(Q^{\alpha}\left(1-\eta_{\alpha, \beta}\right)\right)\right)=T^{\alpha}\left(\frac{1}{G^{\beta}\left(Q^{\alpha}\left(1-\eta_{\alpha, \beta}\right)\right)}\right)=T^{\alpha}\left(\frac{V_{\kappa_{\beta}+1}^{\beta}}{V_{\kappa_{\beta}}^{\beta}}\right)=\tau_{\alpha, \beta},
$$

which justifies (69). According to Lemma 23, we have that $0<\mu_{\alpha, \beta}<1$ and $\mu_{\alpha, \beta} \neq \frac{1}{2}$, which means that $Q^{\alpha}\left(\mu_{\alpha, \beta}\right)<0$ and $Q^{\alpha}\left(\mu_{\alpha, \beta}\right) \neq-1$. As in the previous case, using (39), we simplify $G^{\beta}\left(Q^{\alpha}\left(\mu_{\alpha, \beta}\right)\right)$ as $V_{\kappa_{\beta}+1}^{\beta} / V_{\kappa_{\beta}}^{\beta}$, which justifies (70). Finally, if we combine (69) and (70) and use (27) then we obtain (71).

Let us note that for $0<\alpha<4$, we have

$$
\begin{aligned}
0<\eta_{\alpha, \beta}<\tau_{\alpha, \beta}<\mu_{\alpha, \beta}<\frac{1}{2} & \text { for } \beta>4, \\
0<\eta_{\alpha, \beta}<\tau_{\alpha, \beta}<\mu_{\alpha, \beta}=\frac{1}{2} & \text { for } \beta=4, \\
0<\eta_{\alpha, \beta}<\frac{1}{2}<\mu_{\alpha, \beta}<1 & \text { for } 2<\beta<4,
\end{aligned}
$$

since $Q^{\alpha}\left(\eta_{\alpha, \beta}\right)=1-\beta, Q^{\alpha}\left(\tau_{\alpha, \beta}\right)=2-\beta$ and $Q^{\alpha}\left(\mu_{\alpha, \beta}\right)=\frac{2-\beta}{2}$ for $\beta>2$. The following lemma indicates that the values of $\eta_{\alpha, \beta}$ and $\mu_{\alpha, \beta}$ are separated by $\frac{1}{2}$ for $0<\alpha, \beta<4$.

Lemma 25. For $0<\alpha, \beta<4$, we have that

$$
0 \leq \eta_{\alpha, \beta}<\frac{1}{2} \leq \mu_{\alpha, \beta}<1
$$

Proof. Firstly, for $2<\beta<4$, we have the inequalities in (73). Secondly, for $0<\beta<2$ such that $\beta \neq \xi_{k}, k \in \mathbb{N}, k>2$, we have $0<\tau_{\alpha, \beta}<1$, which implies $G^{\beta}\left(Q^{\alpha}\left(\mu_{\alpha, \beta}\right)\right)<0$ according to (70). Thus, we obtain that $-1<Q^{\alpha}\left(\mu_{\alpha, \beta}\right)<0$, which leads to $\frac{1}{2}<\mu_{\alpha, \beta}<1$. Similarly, using (71), we get $G^{\beta}\left(Q^{\alpha}\left(1-\eta_{\alpha, \beta}\right)\right)<0$, which implies $0<\eta_{\alpha, \beta}<\frac{1}{2}$. Finally, for $\beta=\xi_{k}, k \in \mathbb{N}, k \geq 2$, we have $\eta_{\alpha, \beta}=0$ and $\mu_{\alpha, \beta}=\frac{1}{2}$ due to Lemma 23 .

Proof of Theorem 5. The proof is based on Theorem 26 in [20]. Let us find the description of the Fuč́k curve $\mathcal{C}_{2 j-1}^{+}, j \in \mathbb{N}$, in terms of functions $t_{j}^{+}=t_{j}^{+}(\alpha, \beta)$ and $t_{j}^{-}=t_{j}^{-}(\alpha, \beta)$ defined in (24) and (25), respectively. Using Theorem 26 in [20], we obtain that the Fučík curve $\mathcal{C}_{2 j-1}^{+}$has in $\mathcal{D}$ the following implicit description

$$
\sum_{i=1}^{j} p_{i}(\alpha, \beta)+\sum_{i=1}^{j} p_{i}(\beta, \alpha)+T^{\alpha}\left(\vartheta_{j}(\alpha, \beta)\right)+T^{\alpha}\left(\vartheta_{j}(\beta, \alpha)\right)=n+1
$$

and moreover, the corresponding non-trivial solution $u$ has exactly $(2 j-1)$ generalized zeros on $\mathbb{T}$ and has exactly $j$ positive semi-waves as continuous extensions. These positive continuous semi-waves have the zeros $t_{i}$ and $\tilde{t}_{-i}$, which can be reconstructed from left and right endpoints of $\widehat{\mathbb{T}}$, respectively, in the following way 


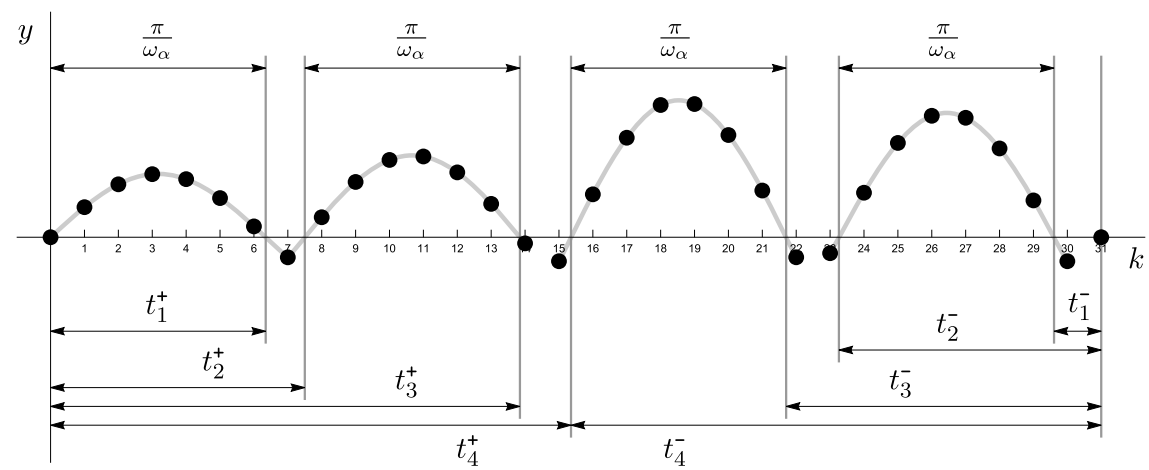

Fig. 29. A non-trivial solution of the problem (7) with 7 generalized zeros on $\mathbb{T}$ for $(\alpha, \beta) \in \mathcal{C}_{7}^{+}(n=30$, $\alpha \doteq 0.240, \beta \doteq 3.534)$.

$$
t_{i}=\sum_{k=1}^{i} p_{k}(\alpha, \beta)+T^{\alpha}\left(\vartheta_{i}(\alpha, \beta)\right), \quad \tilde{t}_{-i}=n+1-\sum_{k=1}^{i} p_{k}(\beta, \alpha)-T^{\alpha}\left(\vartheta_{i}(\beta, \alpha)\right),
$$

for $i=1, \ldots, j$. The condition (74) means that $t_{j}=\tilde{t}_{-j}$. Now, according to Lemma 14 , Remark 18 and (66), we have that

$$
\begin{aligned}
t_{i} & =t_{i}^{+}(\alpha, \beta), \\
\tilde{t}_{-i} & =n+1-t_{i}^{-}(\alpha, \beta),
\end{aligned}
$$

and thus, the condition (74) reads as

$$
t_{j}^{+}(\alpha, \beta)+t_{j}^{-}(\alpha, \beta)=n+1
$$

To justify (75), it remains to show that $\varrho_{\alpha, \beta}(0)=p_{1}(\beta, \alpha)+T^{\alpha}\left(\vartheta_{1}(\beta, \alpha)\right)$. Indeed, using Lemma 20, we obtain

$$
\varrho_{\alpha, \beta}(0)=\kappa_{\beta}+\mathcal{N}_{\alpha, \beta}(0)=\kappa_{\beta}+1+\tau_{\alpha, \beta},
$$

and using Lemma 12, we get

$$
\begin{aligned}
p_{1}(\beta, \alpha) & =\kappa_{\beta}+1 \\
T^{\alpha}\left(\vartheta_{1}(\beta, \alpha)\right) & =T^{\alpha}\left(W_{p_{1}(\beta, \alpha)}^{\beta}(\infty)\right)=T^{\alpha}\left(\frac{V_{\kappa_{\beta}+1}^{\beta}}{V_{\kappa_{\beta}}^{\beta}}\right)=\tau_{\alpha, \beta} .
\end{aligned}
$$

Now, the description of Fučík curves $\mathcal{C}_{2 j-1}^{-}, \mathcal{C}_{2 j}^{+}$and $\mathcal{C}_{2 j}^{-}$in terms of functions $t_{j}^{+}=$ $t_{j}^{+}(\alpha, \beta)$ and $t_{j}^{-}=t_{j}^{-}(\alpha, \beta)$ can be obtained analogously (see Figs. 30 and 31$)$. Let us only mention here the implicit description of curves $\mathcal{C}_{2 j}^{ \pm}$similar to (74)

$$
\mathcal{C}_{2 j}^{+}: \sum_{i=1}^{j+1} p_{i}(\alpha, \beta)+\sum_{i=1}^{j} p_{i}(\alpha, \beta)+T^{\alpha}\left(\vartheta_{j+1}(\alpha, \beta)\right)+T^{\alpha}\left(\vartheta_{j}(\alpha, \beta)\right)=n+1
$$




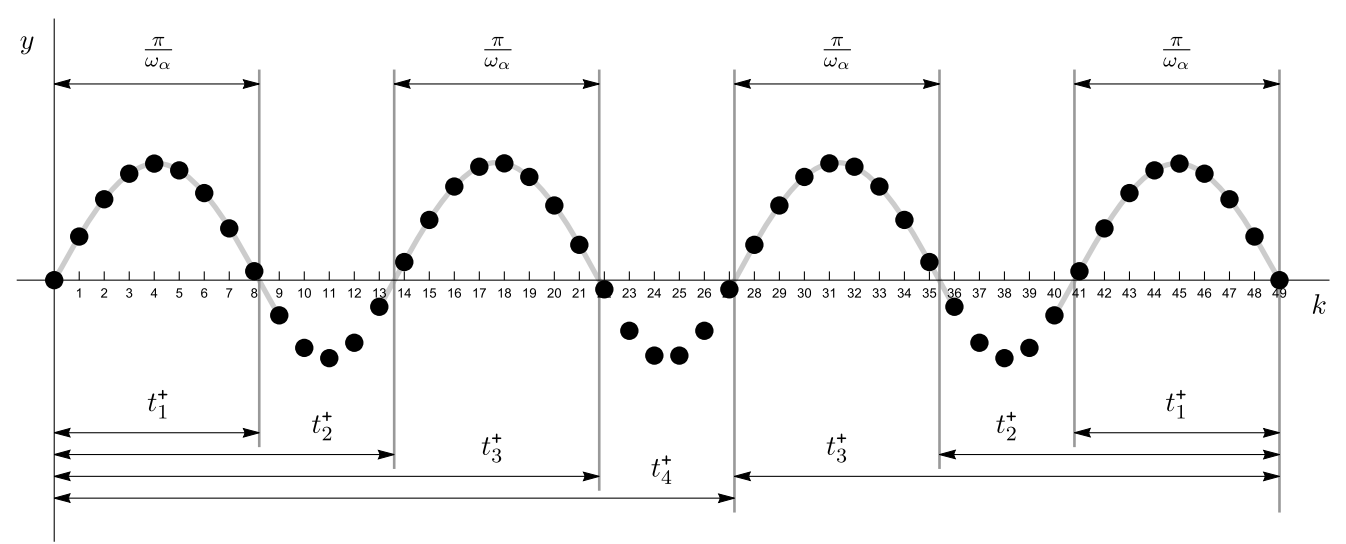

Fig. 30. A non-trivial solution of the problem (7) with 6 generalized zeros on $\mathbb{T}$ for $(\alpha, \beta) \in \mathcal{C}_{6}^{+}(n=48$, $\alpha \doteq 0.145, \beta \doteq 0.329)$.

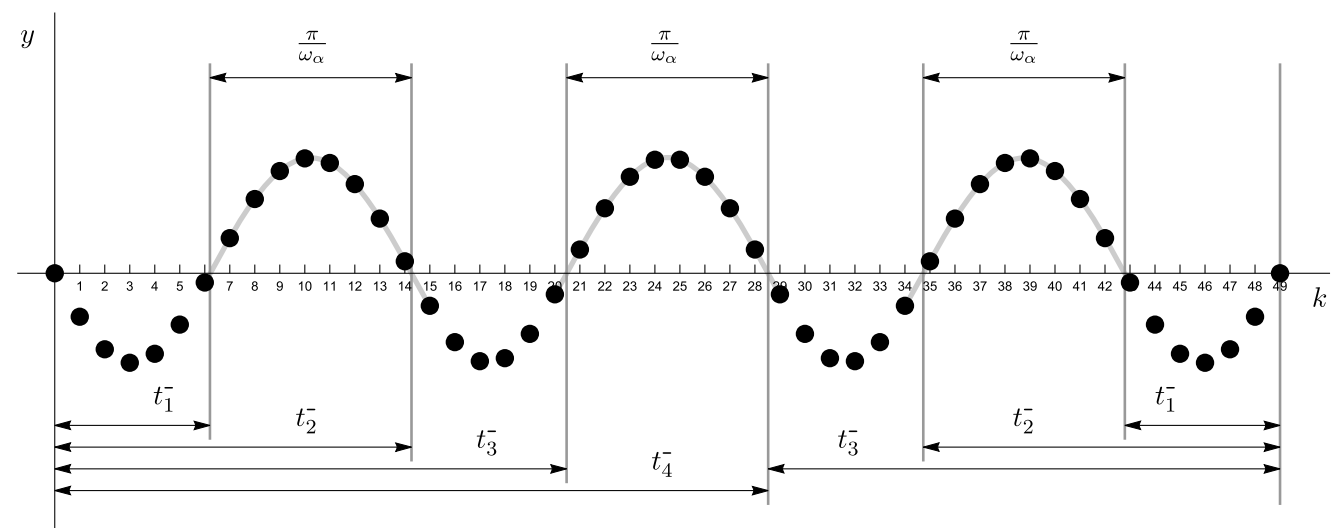

Fig. 31. A non-trivial solution of the problem (7) with 6 generalized zeros on $\mathbb{T}$ for $(\alpha, \beta) \in \mathcal{C}_{6}^{-}(n=48$, $\alpha \doteq 0.150, \beta \doteq 0.251)$.

$$
\mathcal{C}_{2 j}^{-}: \sum_{i=1}^{j+1} p_{i}(\beta, \alpha)+\sum_{i=1}^{j} p_{i}(\beta, \alpha)+T^{\alpha}\left(\vartheta_{j+1}(\beta, \alpha)\right)+T^{\alpha}\left(\vartheta_{j}(\beta, \alpha)\right)=n+1
$$

\section{Improved bounds for Fučík curves}

In this last section, we focus on the function $\rho_{\alpha, \beta}$ introduced in Definition 19 which measures the distance between every two consecutive zeros of two different continuous positive semi-waves. In Theorem 31, we prove that $\rho_{\alpha, \beta}$ attains its global extrema at $\eta_{\alpha, \beta}$ and $\mu_{\alpha, \beta}$ (see Fig. 32). Since $\rho_{\alpha, \beta}$ is defined by $\mathcal{N}_{\alpha, \beta}$, we express the first derivative $\left(\mathcal{N}_{\alpha, \beta}\right)^{\prime}$ in Lemma 28 and then in Lemma 30, we determine where this derivative is less or greater than -1 . Let us note that at the end of this section, the proof of the main Theorem 3 is available. 


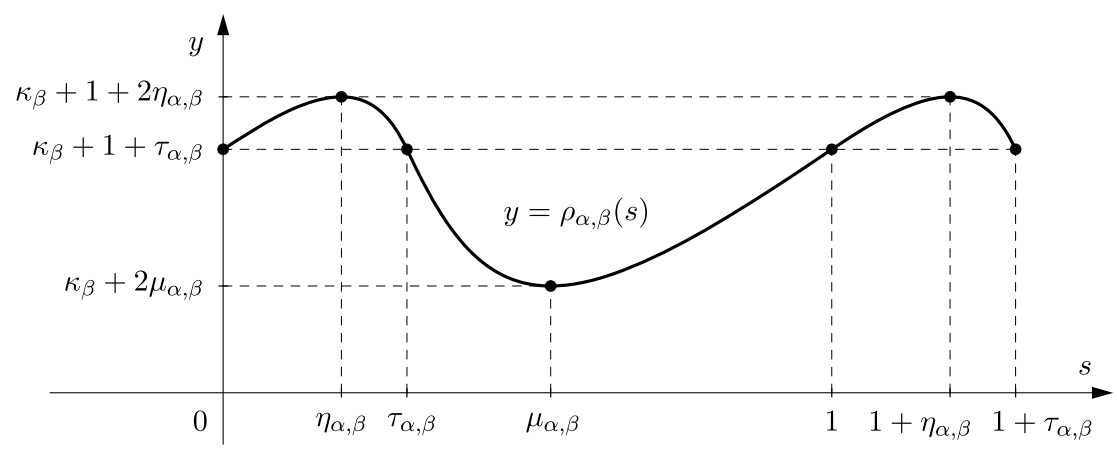

Fig. 32. The graph of the function $\rho_{\alpha, \beta}=\rho_{\alpha, \beta}(s)$ for fixed $\alpha=2.6$ and $\beta=3.8$.

Let us introduce the function $\mathcal{S}_{k}^{\alpha, \beta}$, which we use to express the first derivative $\left(\mathcal{N}_{\alpha, \beta}\right)^{\prime}$ of the function $\mathcal{N}_{\alpha, \beta}$ given in Definition 17.

Definition 26. Let $0<\alpha<4$ and $\beta>0, k \in \mathbb{Z}$. Let us define the function $\mathcal{S}_{k}^{\alpha, \beta}: \mathbb{R}^{*} \rightarrow \mathbb{R}$ as

$$
\begin{aligned}
\mathcal{S}_{k}^{\alpha, \beta}(q) & :=V_{k}^{\beta} \cdot \frac{q^{2} V_{k+1}^{\beta}-2 q V_{k}^{\beta}+V_{k-1}^{\beta}}{q^{2}-(2-\alpha) q+1} \text { for } q \in \mathbb{R}, \\
\mathcal{S}_{k}^{\alpha, \beta}(\infty) & :=V_{k}^{\beta} \cdot V_{k+1}^{\beta} .
\end{aligned}
$$

Let us note that the denominator $q^{2}-(2-\alpha) q+1$ in (76) is always positive since its discriminant is $\alpha(\alpha-4)<0$.

Lemma 27. Let $0<\alpha<4, \beta>0$ and $k=\kappa_{\beta}$ or $k=\kappa_{\beta}+1$. Then for $0<t \leq 1$, we have

$$
\left(T^{\alpha} \circ W_{k+1}^{\beta} \circ Q^{\alpha}\right)^{\prime}(t)=\frac{1}{1-(\beta-\alpha) \mathcal{S}_{k}^{\alpha, \beta}\left(Q^{\alpha}(t)\right)} .
$$

Moreover, we have

$$
\left(T^{\alpha} \circ W_{k+1}^{\beta} \circ Q^{\alpha}\right)_{+}^{\prime}(0)=\frac{1}{1-(\beta-\alpha) \mathcal{S}_{k}^{\alpha, \beta}(\infty)} .
$$

Proof. For $0 \leq t \leq 1$, let us denote $q=Q^{\alpha}(t)$. Thus, we have that $q$ is finite and non-positive for $0<t \leq 1$ and $q=\infty$ for $t=0$.

At first, in the special case of $V_{k}^{\beta}=0$, we have that $\mathcal{S}_{k}^{\alpha, \beta}(q)=0$ and $W_{k+1}^{\beta}(q)=q$ and thus, we obtain

$$
T^{\alpha}\left(W_{k+1}^{\beta}\left(Q^{\alpha}(t)\right)\right)=T^{\alpha}\left(Q^{\alpha}(t)\right)=t
$$


which justifies (78) and (79).

Now, for the rest of the proof, let us assume that $V_{k}^{\beta} \neq 0$. Using (12), we obtain

$$
\begin{aligned}
\left(T^{\alpha}\right)^{\prime}(q) & =\frac{\sin \omega_{\alpha}}{\omega_{\alpha}} \cdot \frac{1}{q^{2}-(2-\alpha) q+1}, \\
\left(Q^{\alpha}\right)^{\prime}(t) & =\frac{1}{\left(T^{\alpha}\right)^{\prime}(q)} .
\end{aligned}
$$

Moreover, we have

$$
\left(T^{\alpha}\left(W_{k+1}^{\beta}\left(Q^{\alpha}(t)\right)\right)\right)^{\prime}=\frac{\left(T^{\alpha}\right)^{\prime}\left(W_{k+1}^{\beta}(q)\right)}{\left(T^{\alpha}\right)^{\prime}(q)} \cdot\left(W_{k+1}^{\beta}\right)^{\prime}(q) .
$$

Let us point out that $W_{k+1}^{\beta}(q)$ is a finite number due to $q=Q^{\alpha}(t)$ for $0 \leq t \leq 1$ and $k=\kappa_{\beta}$ or $k=\kappa_{\beta}+1$. Now, let us expand the factor $\left(T^{\alpha}\right)^{\prime}\left(W_{k+1}^{\beta}(q)\right)$ in (81). Thus, let us write the denominator in (80) for $q$ equal to $W_{k+1}^{\beta}(q)$ as

$$
\left(W_{k+1}^{\beta}(q)\right)^{2}-(2-\alpha) W_{k+1}^{\beta}(q)+1=\frac{A q^{2}+B q+C}{\left(q V_{k}^{\beta}-V_{k-1}^{\beta}\right)^{2}},
$$

where the polynomial $A q^{2}+B q+C$ has the form of

$$
\left(q V_{k+1}^{\beta}-V_{k}^{\beta}\right)^{2}-(2-\alpha)\left(q V_{k+1}^{\beta}-V_{k}^{\beta}\right)\left(q V_{k}^{\beta}-V_{k-1}^{\beta}\right)+\left(q V_{k}^{\beta}-V_{k-1}^{\beta}\right)^{2} .
$$

Moreover, the coefficients $A, B$ and $C$ of this polynomial can be identified as

$$
\begin{aligned}
A & =V_{k+1}^{\beta} V_{k+1}^{\beta}-(2-\alpha) V_{k}^{\beta} V_{k+1}^{\beta}+V_{k}^{\beta} V_{k}^{\beta}=1+(2-\beta) V_{k}^{\beta} V_{k+1}^{\beta}-(2-\alpha) V_{k}^{\beta} V_{k+1}^{\beta} \\
& =1-(\beta-\alpha) V_{k}^{\beta} V_{k+1}^{\beta}, \\
C & =V_{k}^{\beta} V_{k}^{\beta}-(2-\alpha) V_{k}^{\beta} V_{k-1}^{\beta}+V_{k-1}^{\beta} V_{k-1}^{\beta}=1+(2-\beta) V_{k}^{\beta} V_{k-1}^{\beta}-(2-\alpha) V_{k}^{\beta} V_{k-1}^{\beta} \\
& =1-(\beta-\alpha) V_{k}^{\beta} V_{k-1}^{\beta}, \\
B & =-2 V_{k}^{\beta}\left(V_{k+1}^{\beta}+V_{k-1}^{\beta}\right)+(2-\alpha)\left(V_{k}^{\beta} V_{k}^{\beta}-V_{k+1}^{\beta} V_{k-1}^{\beta}\right) \\
& =-2(2-\beta) V_{k}^{\beta} V_{k}^{\beta}+(2-\alpha)\left(2 V_{k}^{\beta} V_{k}^{\beta}-1\right) \\
& =-(2-\alpha)+2(\beta-\alpha) V_{k}^{\beta} V_{k}^{\beta} .
\end{aligned}
$$

If we combine (83), (84), (85) and (82), we obtain 


$$
\begin{aligned}
\frac{\left(T^{\alpha}\right)^{\prime}\left(W_{k+1}^{\beta}(q)\right)}{\left(T^{\alpha}\right)^{\prime}(q)} & =\frac{\left(q^{2}-(2-\alpha) q+1\right)\left(q V_{k}^{\beta}-V_{k-1}^{\beta}\right)^{2}}{q^{2}-(2-\alpha) q+1-(\beta-\alpha) V_{k}^{\beta}\left(q^{2} V_{k+1}^{\beta}-2 q V_{k}^{\beta}+V_{k-1}^{\beta}\right)} \\
& =\frac{\left(q V_{k}^{\beta}-V_{k-1}^{\beta}\right)^{2}}{1-(\beta-\alpha) \mathcal{S}_{k}^{\alpha, \beta}(q)},
\end{aligned}
$$

which means that (81) has the form of (78) since we have that

$$
\left(W_{k+1}^{\beta}\right)^{\prime}(q)=\frac{V_{k+1}^{\beta}\left(q V_{k}^{\beta}-V_{k-1}^{\beta}\right)-\left(q V_{k+1}^{\beta}-V_{k}^{\beta}\right) V_{k}^{\beta}}{\left(q V_{k}^{\beta}-V_{k-1}^{\beta}\right)^{2}}=\frac{1}{\left(q V_{k}^{\beta}-V_{k-1}^{\beta}\right)^{2}} .
$$

Using the function $\mathcal{S}_{k}^{\alpha, \beta}$, we express the first derivative $\left(\mathcal{N}_{\alpha, \beta}\right)^{\prime}$ (see Fig. 33).

Lemma 28. For $0<\alpha<4$ and $\beta>0$, we have

$$
\left(\mathcal{N}_{\alpha, \beta}\right)^{\prime}(s)= \begin{cases}\frac{-1}{1-(\beta-\alpha) \mathcal{S}_{\kappa_{\beta}+1}^{\alpha, \beta}\left(Q^{\alpha}(1-s)\right)} & \text { for } s \in\left(0, \tau_{\alpha, \beta}\right], \\ \frac{-1}{1-(\beta-\alpha) \mathcal{S}_{\kappa_{\beta}}^{\alpha, \beta}\left(Q^{\alpha}(1-s)\right)} & \text { for } s \in\left(\tau_{\alpha, \beta}, 1\right), \\ \frac{-1}{1-(\beta-\alpha) \mathcal{S}_{\kappa_{\beta}}^{\alpha, \beta}(\infty)} & \text { for } s=1,\end{cases}
$$

where the functions $\mathcal{S}_{\kappa_{\beta}, \beta}^{\alpha, \beta}$ and $\mathcal{S}_{\kappa_{\beta}+\beta}^{\alpha, \beta}$ are defined in (76), (77) as $\mathcal{S}_{k}^{\alpha, \beta}$ for $k=\kappa_{\beta}$ and $k=\kappa_{\beta}+1$, respectively.

Proof. Let us split the proof according to the value of the variable $s$.

At first, let us assume that $0<s<\tau_{\alpha, \beta}$. Then we have

$$
\mathcal{N}_{\alpha, \beta}(s)=\overline{\bar{M}}_{\alpha, \beta}(s)+1=T^{\alpha}\left(W_{\kappa_{\beta}+2}^{\beta}\left(Q^{\alpha}(1-s)\right)\right)+1
$$

and thus, the expression of the first derivative $\left(\mathcal{N}_{\alpha, \beta}\right)^{\prime}(s)$ follows directly from (78) for $t=1-s$ and $k=\kappa_{\beta}+1$.

At second, let us assume that $\tau_{\alpha, \beta}<s<1$. Then we have

$$
\mathcal{N}_{\alpha, \beta}(s)=\bar{M}_{\alpha, \beta}(s)=T^{\alpha}\left(W_{\kappa_{\beta}+1}^{\beta}\left(Q^{\alpha}(1-s)\right)\right)
$$

and thus, the expression of $\left(\mathcal{N}_{\alpha, \beta}\right)^{\prime}(s)$ follows from (78) for $t=1-s$ and $k=\kappa_{\beta}$.

At third, let us assume that $s=\tau_{\alpha, \beta}$. If we take into account that

$$
V_{\kappa_{\beta}+2}^{\beta} V_{\kappa_{\beta}}^{\beta}-\left(V_{\kappa_{\beta}+1}^{\beta}\right)^{2}=1=V_{\kappa_{\beta}+1}^{\beta} V_{\kappa_{\beta}-1}^{\beta}-\left(V_{\kappa_{\beta}}^{\beta}\right)^{2}
$$


then we obtain

$$
\begin{aligned}
\mathcal{S}_{\kappa_{\beta}+1}^{\alpha, \beta}\left(Q^{\alpha}\left(1-\tau_{\alpha, \beta}\right)\right) & =\mathcal{S}_{\kappa_{\beta}+1}^{\alpha, \beta}\left(\frac{V_{\kappa_{\beta}}^{\beta}}{V_{\kappa_{\beta}+1}^{\beta}}\right) \\
& =V_{\kappa_{\beta}+1}^{\beta} V_{\kappa_{\beta}}^{\beta} \frac{V_{\kappa_{\beta}+2}^{\beta} V_{\kappa_{\beta}}^{\beta}-\left(V_{\kappa_{\beta}+1}^{\beta}\right)^{2}}{\left(V_{\kappa_{\beta}}^{\beta}\right)^{2}-(2-\alpha) V_{\kappa_{\beta}}^{\beta} V_{\kappa_{\beta}+2}^{\beta}+\left(V_{\kappa_{\beta}+1}^{\beta}\right)^{2}} \\
& =V_{\kappa_{\beta}+1}^{\beta} V_{\kappa_{\beta}}^{\beta} \frac{V_{\kappa_{\beta}+1}^{\beta} V_{\kappa_{\beta}-1}^{\beta}-\left(V_{\kappa_{\beta}}^{\beta}\right)^{2}}{\left(V_{\kappa_{\beta}}^{\beta}\right)^{2}-(2-\alpha) V_{\kappa_{\beta}}^{\beta} V_{\kappa_{\beta}+2}^{\beta}+\left(V_{\kappa_{\beta}+1}^{\beta}\right)^{2}} \\
& =\mathcal{S}_{\kappa_{\beta}}^{\alpha, \beta}\left(\frac{V_{\kappa_{\beta}}^{\beta}}{V_{\kappa_{\beta}+1}^{\beta}}\right)^{2} \\
& =\mathcal{S}_{\kappa_{\beta}, \beta}^{\alpha, \beta}\left(Q^{\alpha}\left(1-\tau_{\alpha, \beta}\right)\right) .
\end{aligned}
$$

And thus, we obtain that the one-sided derivatives of $\mathcal{N}_{\alpha, \beta}$ at $\tau_{\alpha, \beta}$ coincide. Indeed, we have

$$
\begin{aligned}
\left(\mathcal{N}_{\alpha, \beta}\right)_{-}^{\prime}\left(\tau_{\alpha, \beta}\right) & =\frac{-1}{1-(\beta-\alpha) \mathcal{S}_{\kappa_{\beta}+\beta}^{\alpha, \beta}\left(Q^{\alpha}\left(1-\tau_{\alpha, \beta}\right)\right)}=\frac{-1}{1-(\beta-\alpha) \mathcal{S}_{\kappa_{\beta}}^{\alpha, \beta}\left(Q^{\alpha}\left(1-\tau_{\alpha, \beta}\right)\right)} \\
& =\left(\mathcal{N}_{\alpha, \beta}\right)_{+}^{\prime}\left(\tau_{\alpha, \beta}\right) .
\end{aligned}
$$

Remark 29. If we take into account that $\mathcal{S}_{\kappa_{\beta}}^{\alpha, \beta}(\infty)=\mathcal{S}_{\kappa_{\beta}+1}^{\alpha, \beta}(0)$, we obtain using Lemma 28 that

$$
\left(\mathcal{N}_{\alpha, \beta}\right)^{\prime}(1)=\frac{-1}{1-(\beta-\alpha) \mathcal{S}_{\kappa_{\beta}+1}^{\alpha, \beta}(0)}=\left(\mathcal{N}_{\alpha, \beta}\right)_{+}^{\prime}(0)
$$

Moreover, due to Lemma 20, the function $\mathcal{N}_{\alpha, \beta}$ is an involution and thus, for all $s \in$ $\left(0,1+\tau_{\alpha, \beta}\right)$, we have that $\left(\mathcal{N}_{\alpha, \beta}\right)^{\prime}\left(\mathcal{N}_{\alpha, \beta}(s)\right) \cdot\left(\mathcal{N}_{\alpha, \beta}\right)^{\prime}(s)=1$. And thus, we have

$$
\frac{1}{\left(\mathcal{N}_{\alpha, \beta}\right)^{\prime}\left(\tau_{\alpha, \beta}\right)}=\left(\mathcal{N}_{\alpha, \beta}\right)^{\prime}(1)=\left(\mathcal{N}_{\alpha, \beta}\right)_{+}^{\prime}(0)=\frac{1}{\left(\mathcal{N}_{\alpha, \beta}\right)_{-}^{\prime}\left(1+\tau_{\alpha, \beta}\right)} .
$$

Let us examine where the value of the first derivative $\left(\mathcal{N}_{\alpha, \beta}\right)^{\prime}(s)$ is equal, less or greater than -1 (see Fig. 33).

Lemma 30. Let $0<\alpha<4$ and $\beta>0$. For $\beta=\xi_{k}, k \in \mathbb{N}, k \geq 2$, we have that

$$
\left(\mathcal{N}_{\alpha, \beta}\right)^{\prime}(s)=-1 \quad \text { for } s \in(0,1)
$$




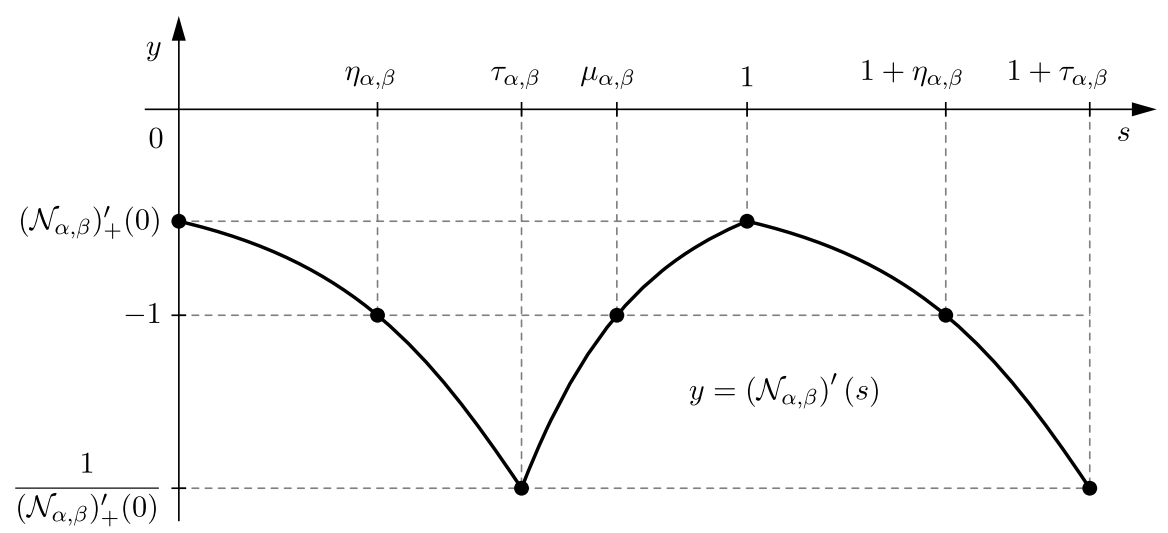

Fig. 33. The graph of the first derivative $s \mapsto\left(\mathcal{N}_{\alpha, \beta}\right)^{\prime}(s)$ for fixed $\alpha=1.5$ and $\beta=2.7$.

In the case of $\beta \neq \xi_{k}$ for all $k \in \mathbb{N}, k \geq 2$, we have for $\alpha \lessgtr \beta$ that

$$
\left(\mathcal{N}_{\alpha, \beta}\right)^{\prime}(s) \begin{cases}\gtrless-1 & \text { for } s \in\left(0, \eta_{\alpha, \beta}\right) \cup\left(\mu_{\alpha, \beta}, 1\right), \\ =-1 & \text { for } s=\eta_{\alpha, \beta} \text { and for } s=\mu_{\alpha, \beta}, \\ \lessgtr-1 & \text { for } s \in\left(\eta_{\alpha, \beta}, \mu_{\alpha, \beta}\right),\end{cases}
$$

and the statement (86) holds for $\alpha=\beta$.

Proof. In the case of $\beta=\xi_{k}, k \in \mathbb{N}, k \geq 2$, we have $\mathcal{N}_{\alpha, \beta}(s)=1-s$ according to Lemma 22 and thus, (86) holds. For the rest of the proof, let us assume that $\beta \neq \xi_{k}$ for all $k \in \mathbb{N}, k \geq 2$. According to Lemma 23, we have that $0<\eta_{\alpha, \beta}<\tau_{\alpha, \beta}<\mu_{\alpha, \beta}<1$. Now, let us denote $q=Q^{\alpha}(1-s)$ and split the proof according to the value of $s$.

At first, let us assume that $\tau_{\alpha, \beta}<s<1$. Thus, we have $q<V_{\kappa_{\beta}}^{\beta} / V_{\kappa_{\beta}+1}^{\beta}<0$. Using Lemma 28, we obtain

$$
\left(\mathcal{N}_{\alpha, \beta}\right)^{\prime}(s) \gtreqless-1 \quad \text { if and only if } \quad(\alpha-\beta) \cdot \mathcal{S}_{\kappa_{\beta}}^{\alpha, \beta}(q) \gtreqless 0 \text {. }
$$

If we take into account that $V_{\kappa_{\beta}-1}^{\beta} V_{\kappa_{\beta}+1}^{\beta}=\left(V_{\kappa_{\beta}}^{\beta}\right)^{2}-1$, it is possible to write $\mathcal{S}_{\kappa_{\beta}}^{\alpha, \beta}(q)$ in the following form

$$
\mathcal{S}_{\kappa_{\beta}}^{\alpha, \beta}(q)=V_{\kappa_{\beta}}^{\beta} V_{\kappa_{\beta}+1}^{\beta} \frac{\left(q-\frac{V_{\kappa_{\beta}}^{\beta}-1}{V_{\kappa_{\beta}+1}^{\beta}}\right)\left(q-\frac{V_{\kappa_{\beta}}^{\beta}+1}{V_{\kappa_{\beta}+1}^{\beta}}\right)}{q^{2}-(2-\alpha) q+1} .
$$

Thus, the sign of $\mathcal{S}_{\kappa_{\beta}}^{\alpha, \beta}(q)$ is equal to the sign of the factor $\left(q-\left(V_{\kappa_{\beta}}^{\beta}+1\right) / V_{\kappa_{\beta}+1}^{\beta}\right)$ due to

$$
V_{\kappa_{\beta}}^{\beta} V_{\kappa_{\beta}+1}^{\beta}<0, \quad q-\frac{V_{\kappa_{\beta}}^{\beta}-1}{V_{\kappa_{\beta}+1}^{\beta}}<0, \quad q^{2}-(2-\alpha) q+1>0 .
$$


Now, since $q=Q^{\alpha}(1-s)$ and $\left(V_{\kappa_{\beta}}^{\beta}+1\right) / V_{\kappa_{\beta}+1}^{\beta}=1 / Q^{\alpha}\left(\mu_{\alpha, \beta}\right)=Q^{\alpha}\left(1-\mu_{\alpha, \beta}\right)$, we conclude that

$$
\mathcal{S}_{\kappa_{\beta}}^{\alpha, \beta}(q) \gtreqless 0 \quad \text { if and only if } \quad Q^{\alpha}(1-s) \gtreqless Q^{\alpha}\left(1-\mu_{\alpha, \beta}\right) \text {. }
$$

If we combine (87) and (88) and take into account that $Q^{\alpha}$ is a strictly increasing function, we obtain

$$
\left(\mathcal{N}_{\alpha, \beta}\right)^{\prime}(s) \gtreqless-1 \quad \text { if and only if } \quad(\alpha-\beta) \cdot\left(\mu_{\alpha, \beta}-s\right) \gtreqless 0 \text {. }
$$

At second, let us assume that $0<s \leq \tau_{\alpha, \beta}$ and $0<\beta<2$. Thus, we have $V_{\kappa_{\beta}}^{\beta} / V_{\kappa_{\beta}+1}^{\beta} \leq$ $q<0$. Using Lemma 28, we obtain

$$
\left(\mathcal{N}_{\alpha, \beta}\right)^{\prime}(s) \gtreqless-1 \quad \text { if and only if } \quad(\alpha-\beta) \cdot \mathcal{S}_{\kappa_{\beta}+1}^{\alpha, \beta}(q) \gtreqless 0 .
$$

It is possible to write $\mathcal{S}_{\kappa_{\beta}+1}^{\alpha, \beta}(q)$ in the following form

$$
\mathcal{S}_{\kappa_{\beta}+1}^{\alpha, \beta}(q)=V_{\kappa_{\beta}+1}^{\beta} \frac{\left(q V_{\kappa_{\beta}+2}^{\beta}-V_{\kappa_{\beta}+1}^{\beta}+1\right)\left(q-\frac{V_{\kappa_{\beta}}^{\beta}}{V_{\kappa_{\beta}+1}^{\beta}-1}\right)}{q^{2}-(2-\alpha) q+1} .
$$

Now, the factor $\left(q V_{\kappa_{\beta}+2}^{\beta}-V_{\kappa_{\beta}+1}^{\beta}+1\right)$ is positive since for $0<\beta<2$, we have

$$
V_{\kappa_{\beta}}^{\beta}>0, \quad V_{\kappa_{\beta}+1}^{\beta}<0, \quad V_{\kappa_{\beta}+2}^{\beta}=(2-\beta) V_{\kappa_{\beta}+1}^{\beta}-V_{\kappa_{\beta}}^{\beta}<0 .
$$

Thus, $\mathcal{S}_{\kappa_{\beta}+1}^{\alpha, \beta}(q)$ has the opposite sign than the factor $\left(q-V_{\kappa_{\beta}}^{\beta} /\left(V_{\kappa_{\beta}+1}^{\beta}-1\right)\right)$. Moreover, since $q=Q^{\alpha}(1-s)$ and $V_{\kappa_{\beta}}^{\beta} /\left(V_{\kappa_{\beta}+1}^{\beta}-1\right)=1 / Q^{\alpha}\left(\eta_{\alpha, \beta}\right)=Q^{\alpha}\left(1-\eta_{\alpha, \beta}\right)$, we conclude that

$$
\mathcal{S}_{\kappa_{\beta}+\beta}^{\alpha, \beta}(q) \gtreqless 0 \quad \text { if and only if } \quad Q^{\alpha}(1-s) \lesseqgtr Q^{\alpha}\left(1-\eta_{\alpha, \beta}\right) \text {. }
$$

If we combine (90) and (92) and take into account that $Q^{\alpha}$ is a strictly increasing function, we obtain

$$
\left(\mathcal{N}_{\alpha, \beta}\right)^{\prime}(s) \gtreqless-1 \quad \text { if and only if } \quad(\alpha-\beta) \cdot\left(\eta_{\alpha, \beta}-s\right) \lesseqgtr 0 \text {. }
$$

At third, let us assume that $0<s \leq \tau_{\alpha, \beta}$ and $\beta>2$. In this case, we have $\kappa_{\beta}=0$, $\frac{1}{2-\beta} \leq q<0$ and (90) holds. Moreover, the factor $\left(q V_{\kappa_{\beta}+2}^{\beta}-V_{\kappa_{\beta}+1}^{\beta}+1\right)$ in (91) reads as $(\beta-1)(q(\beta-3)+1)$ and thus, it is positive due to $0 \leq q(\beta-2)+1$. As in the previous case, we obtain that (93) holds.

Finally, the statement now follows from (89) and (93). 
The following theorem provides the values of the global extrema of the function $\rho_{\alpha, \beta}$ as well as points where these extrema are attained: $\mu_{\alpha, \beta}$ and $\eta_{\alpha, \beta}$.

Theorem 31. Let $0<\alpha<4$ and $\beta>0$. Then the function $\rho_{\alpha, \beta}$ attains its global extrema at $\eta_{\alpha, \beta}$ and $\mu_{\alpha, \beta}$. More precisely, we have that

$$
\begin{array}{r}
\min _{s \in\left[0,1+\tau_{\alpha, \beta}\right]} \rho_{\alpha, \beta}(s)= \begin{cases}\rho_{\alpha, \beta}\left(\mu_{\alpha, \beta}\right)=2 \mu_{\alpha, \beta}+\kappa_{\beta} & \text { for } \alpha \leq \beta, \\
\rho_{\alpha, \beta}\left(\eta_{\alpha, \beta}\right)=2 \eta_{\alpha, \beta}+\kappa_{\beta}+1 & \text { for } \alpha>\beta,\end{cases} \\
\max _{s \in\left[0,1+\tau_{\alpha, \beta}\right]} \rho_{\alpha, \beta}(s)= \begin{cases}\rho_{\alpha, \beta}\left(\eta_{\alpha, \beta}\right)=2 \eta_{\alpha, \beta}+\kappa_{\beta}+1 & \text { for } \alpha \leq \beta, \\
\rho_{\alpha, \beta}\left(\mu_{\alpha, \beta}\right)=2 \mu_{\alpha, \beta}+\kappa_{\beta} & \text { for } \alpha>\beta .\end{cases}
\end{array}
$$

Proof. Using Lemma 28 and Remark 29, we get that the function $\rho_{\alpha, \beta}$ is continuously differentiable on $\left[0,1+\tau_{\alpha, \beta}\right]$. Let us point out that $\rho_{\alpha, \beta}(s)=\rho(s-1)$ for $1 \leq s \leq 1+\tau_{\alpha, \beta}$. Indeed, for $1 \leq s \leq 1+\tau_{\alpha, \beta}$, we have

$$
\begin{aligned}
\rho_{\alpha, \beta}(s) & =s+\kappa_{\beta}+\overline{\bar{M}}_{\alpha, \beta}(s-1)=s-1+\kappa_{\beta}+\overline{\bar{M}}_{\alpha, \beta}(s-1)+1 \\
& =(s-1)+\kappa_{\beta}+\mathcal{N}_{\alpha, \beta}(s-1)=\rho_{\alpha, \beta}(s-1) .
\end{aligned}
$$

Thus, we obtain for $0<s<1+\tau_{\alpha, \beta}$ that

$$
\left(\rho_{\alpha, \beta}\right)^{\prime}(s)=1+\left(\mathcal{N}_{\alpha, \beta}\right)^{\prime}(s)
$$

Moreover, using Lemma 21 we have that

$$
\begin{aligned}
& \rho_{\alpha, \beta}\left(\eta_{\alpha, \beta}\right)=\eta_{\alpha, \beta}+\kappa_{\beta}+\mathcal{N}_{\alpha, \beta}\left(\eta_{\alpha, \beta}\right)=2 \eta_{\alpha, \beta}+\kappa_{\beta}+1, \\
& \rho_{\alpha, \beta}\left(\mu_{\alpha, \beta}\right)=\mu_{\alpha, \beta}+\kappa_{\beta}+\mathcal{N}_{\alpha, \beta}\left(\mu_{\alpha, \beta}\right)=2 \mu_{\alpha, \beta}+\kappa_{\beta} .
\end{aligned}
$$

For $\beta=\xi_{k}, k \in \mathbb{N}, k \geq 2$, we have that $\mathcal{N}_{\alpha, \beta}(s)=1-s$ according to Lemma 22 and thus $\rho_{\alpha, \beta}(s) \equiv 1+\kappa_{\beta}$, which means that $\rho_{\alpha, \beta}$ is a constant function. Let us note that in this case, we have $\eta_{\alpha, \beta}=0$ and $\mu_{\alpha, \beta}=\frac{1}{2}$ due to Lemma 23.

Now, let us assume that $\beta \neq \xi_{k}$ for all $k \in \mathbb{N}, k \geq 2$. Using Lemma 30, we determine the monotonic intervals of $\rho_{\alpha, \beta}$. The points $\mu_{\alpha, \beta}$ and $\eta_{\alpha, \beta}$ are stationary points of $\rho_{\alpha, \beta}$. Firstly, let us assume that $\alpha<\beta$. Then $\rho_{\alpha, \beta}$ is strictly increasing on intervals $\left(0, \eta_{\alpha, \beta}\right)$, $\left(\mu_{\alpha, \beta}, 1\right)$ and strictly decreasing on the interval $\left(\eta_{\alpha, \beta}, \mu_{\alpha, \beta}\right)$. Thus, $\eta_{\alpha, \beta}$ and $\mu_{\alpha, \beta}$ are points of the global extrema of $\rho_{\alpha, \beta}$ on the interval $\left[0,1+\tau_{\alpha, \beta}\right]$ (the global maximum and the global minimum, respectively). Secondly, let us assume that $\alpha>\beta$. In this case, we similarly obtain that $\eta_{\alpha, \beta}$ and $\mu_{\alpha, \beta}$ are points of the global minimum and the global maximum, respectively. Finally, in the case of $\alpha=\beta$, we get using Lemma 22 that $\mathcal{N}_{\alpha, \beta}(s)=1-s+\tau_{\beta, \beta}=1-s+\frac{\pi}{\omega_{\beta}}-\left\lfloor\frac{\pi}{\omega_{\beta}}\right\rfloor$. Thus, we obtain that $\rho_{\alpha, \beta}$ is a constant function such that $\rho_{\alpha, \beta}(s) \equiv 1+\kappa_{\beta}+\tau_{\beta, \beta}=\frac{\pi}{\omega_{\beta}}$. Let us note that $\tau_{\beta, \beta}=2 \eta_{\beta, \beta}=2 \mu_{\beta, \beta}-1$ in this case. 
Remark 32. Due to Theorem 31, we have for all $(\alpha, \beta) \in \mathcal{D}$ and $s \in\left[0,1+\tau_{\alpha, \beta}\right]$ that $\rho_{\alpha, \beta}^{\min } \leq \rho_{\alpha, \beta}(s) \leq \rho_{\alpha, \beta}^{\max }$, where $\rho_{\alpha, \beta}^{\min }$ and $\rho_{\alpha, \beta}^{\max }$ are given in Definition 2. Moreover, using Lemma 25, we get the following bounds

$$
\left\lfloor\frac{\pi}{\omega_{\beta}}\right\rfloor=1+\kappa_{\beta} \leq \rho_{\alpha, \beta}^{\min } \leq \rho_{\alpha, \beta}(s) \leq \rho_{\alpha, \beta}^{\max }<2+\kappa_{\beta}=\left\lfloor\frac{\pi}{\omega_{\beta}}\right\rfloor+1
$$

for $0<\alpha, \beta<4$.

Proof of Theorem 3. At first, let us prove that $\left(\mathcal{C}_{2 j-1}^{ \pm} \cap \mathcal{D}\right) \subset \Upsilon_{2 j-1}^{ \pm}, j \in \mathbb{N}$, where

$$
\Upsilon_{2 j-1}^{ \pm}=\left\{(\alpha, \beta) \in \mathcal{D}: \rho_{\alpha, \beta}^{\min } \leq \frac{n+1}{j}-\frac{\pi}{\omega_{\alpha}} \leq \rho_{\alpha, \beta}^{\max }\right\}
$$

Thus, let us assume that $(\alpha, \beta) \in \mathcal{C}_{2 j-1}^{ \pm} \cap \mathcal{D}$. Then using Theorem 5 , we get

$$
t_{j}^{+}(\alpha, \beta)+t_{j}^{-}(\alpha, \beta)=n+1
$$

where $t_{j}^{+}$and $t_{j}^{-}$are defined in (24) and (25), respectively. The corresponding non-trivial solution $u$ consists of $j$ positive and $j$ negative semi-waves (as continuous extensions) and the equation (94) reads as

$$
j \cdot \frac{\pi}{\omega_{\alpha}}+\sum_{i=1}^{j} \rho_{\alpha, \beta}\left(\left\lceil t_{2 i-1}\right\rceil-t_{2 i-1}\right)=n+1,
$$

where $t_{i}, i=1, \ldots, 2 j-1$, are zeros of positive semi-waves

$$
0<t_{1}=t_{1}^{+}<\cdots<t_{j}=t_{j}^{+}=n+1-t_{j}^{-}<\cdots<t_{2 j-1}=n+1-t_{1}^{-}<n+1 .
$$

Now, using Theorem 31, we obtain for $i=1, \ldots, j$ that

$$
\begin{aligned}
2 \mu_{\alpha, \beta}+\kappa_{\beta} \leq \rho_{\alpha, \beta}\left(\left\lceil t_{2 i-1}\right\rceil-t_{2 i-1}\right) & \leq 2 \eta_{\alpha, \beta}+\kappa_{\beta}+1 & & \text { for } \alpha \leq \beta, \\
1+2 \eta_{\alpha, \beta}+\kappa_{\beta} \leq \rho_{\alpha, \beta}\left(\left\lceil t_{2 i-1}\right\rceil-t_{2 i-1}\right) & \leq 2 \mu_{\alpha, \beta}+\kappa_{\beta} & & \text { for } \alpha>\beta .
\end{aligned}
$$

And thus, we have

$$
j \cdot \rho_{\alpha, \beta}^{\min } \leq \sum_{i=1}^{j} \rho_{\alpha, \beta}\left(\left\lceil t_{2 i-1}\right\rceil-t_{2 i-1}\right) \leq j \cdot \rho_{\alpha, \beta}^{\max },
$$

where $\rho_{\alpha, \beta}^{\min }$ and $\rho_{\alpha, \beta}^{\max }$ are given in Definition 2. Finally, if we combine (95) and (96), we obtain $\rho_{\alpha, \beta}^{\min } \leq \frac{n+1}{j}-\frac{\pi}{\omega_{\alpha}} \leq \rho_{\alpha, \beta}^{\max }$.

At second, let us show that $\left(\mathcal{C}_{2 j}^{+} \cap \mathcal{D}\right) \subset \Upsilon_{2 j}^{+}, j \in \mathbb{N}$, where

$$
\Upsilon_{2 j}^{+}=\left\{(\alpha, \beta) \in \mathcal{D}: \rho_{\alpha, \beta}^{\min } \leq \frac{n+1}{j}-\frac{j+1}{j} \cdot \frac{\pi}{\omega_{\alpha}} \leq \rho_{\alpha, \beta}^{\max }\right\}
$$


Using Theorem 5, we obtain for $(\alpha, \beta) \in \mathcal{C}_{2 j}^{+} \cap \mathcal{D}$ that

$$
t_{j+1}^{+}(\alpha, \beta)+t_{j}^{+}(\alpha, \beta)=n+1
$$

and the corresponding non-trivial solution $u$ consists of $(j+1)$ positive and $j$ negative semi-waves. The equation (97) can be also written as

$$
(j+1) \cdot \frac{\pi}{\omega_{\alpha}}+\sum_{i=1}^{j} \rho_{\alpha, \beta}\left(\left\lceil t_{2 i-1}\right\rceil-t_{2 i-1}\right)=n+1,
$$

where $t_{i}, i=1, \ldots, 2 j-1$, are zeros of positive semi-waves. Using Theorem 31, we obtain the same inequalities as in (96). Now, if we combine (96) and (98), we get $\rho_{\alpha, \beta}^{\min } \leq$ $\frac{n+1}{j}-\frac{j+1}{j} \cdot \frac{\pi}{\omega_{\alpha}} \leq \rho_{\alpha, \beta}^{\max }$.

At third, the last type of the inclusion $\left(\mathcal{C}_{2 j}^{-} \cap \mathcal{D}\right) \subset \Upsilon_{2 j}^{-}, j \in \mathbb{N}$, can be proved similarly as in the previous two cases. Let us only note that for $(\alpha, \beta) \in \mathcal{C}_{2 j}^{-} \cap \mathcal{D}$, we obtain $\left(t_{0}=0\right)$

$$
j \cdot \frac{\pi}{\omega_{\alpha}}+\sum_{i=0}^{j} \rho_{\alpha, \beta}\left(\left\lceil t_{2 i}\right\rceil-t_{2 i}\right)=n+1,
$$

which leads to $\rho_{\alpha, \beta}^{\min } \leq \frac{n+1}{j+1}-\frac{j}{j+1} \cdot \frac{\pi}{\omega_{\alpha}} \leq \rho_{\alpha, \beta}^{\max }$.

\section{Conclusion}

In the paper, we improve and extend known results for the Fučík spectrum of the discrete Dirichlet operator. In Theorem 5, we present a new simple implicit description of all non-trivial Fučík curves $\mathcal{C}_{l}^{ \pm}$. Moreover, for each non-trivial Fučík curve $\mathcal{C}_{l}^{ \pm}$, we provide the suitable bound $\Upsilon_{l}^{ \pm}$by two simple curves in Theorem 3. These results are based on Lemma 10 concerning the detailed analysis of nodal properties of the solution $u$ of the discrete initial value problem (30). Generalized zeros of the solution $u$ can be described by the sequence of functions $p_{i}$ which are given recurrently and were introduced in [20]. In Lemma 12, we provide a new simpler expression of these functions $p_{i}$, which can be used to obtain the basic bound $\Theta_{l}^{ \pm}$for each non-trivial Fučík curve $\mathcal{C}_{l}^{ \pm} \subset \Theta_{l}^{ \pm}$(see Theorem 13).

In this paper, we mainly focus on positive semi-waves of $u$ as continuous extensions and investigate the distribution of zeros of these extensions with respect to the integer lattice. More precisely, if $t_{1}$ and $t_{2}$ are two consecutive zeros of two different positive semi-waves (as continuous extensions) then we have

$$
t_{2}=t_{1}+\rho_{\alpha, \beta}\left(\left\lceil t_{1}\right\rceil-t_{1}\right)
$$

where the function $\rho_{\alpha, \beta}$ is given explicitly in Definition 19 using Chebyshev polynomials of the second kind. We use this function $\rho_{\alpha, \beta}$ in Theorem 5 to describe implicitly all 
non-trivial Fučík curves $\mathcal{C}_{l}^{ \pm}$. Let us emphasize that this new description using $\rho_{\alpha, \beta}$ does not require complicated construction of sequences of functions $p_{i}$ and $\vartheta_{i}$ as it was done in $[20]$.

Let us note that in the case of $\alpha=\beta$, the discrete initial value problem (30) is linear and $\rho_{\alpha, \beta}$ is the constant function $\rho_{\alpha, \beta}(s) \equiv \frac{\pi}{\omega_{\beta}}$. Now, for $0<\alpha<4$ and $\beta>0$, the function $\rho_{\alpha, \beta}$ is a differentiable bounded function and its global extrema are given in Theorem 31. Since the global extrema of $\rho_{\alpha, \beta}$ are available in an explicit form, we provide the improved bound $\Upsilon_{l}^{ \pm}$for $\mathcal{C}_{l}^{ \pm}$in Theorem 3 with the boundary given by two simple curves, which are described similarly to the first non-trivial Fučík curve $\mathcal{C}_{1}^{ \pm}$.

\section{Declaration of competing interest}

The authors declare that there is no competing interest.

\section{Acknowledgement}

The authors were supported by the Grant Agency of the Czech Republic, grant no. 18-03253S.

\section{References}

[1] M. Arias, J. Campos, Radial Fučík spectrum of the Laplace operator, J. Math. Anal. Appl. 190 (3) (1995) 654-666.

[2] J. Benedikt, P. Drábek, P. Girg, The first nontrivial curve in the Fuč́k spectrum of the Dirichlet Laplacian on the ball consists of nonradial eigenvalues, Bound. Value Probl. 2011 (9) (2011) 27.

[3] J. Campos, E.N. Dancer, On the resonance set in a fourth-order equation with jumping nonlinearity, Differ. Integral Equ. 14 (3) (2001) 257-272.

[4] E.C.M. Crooks, E.N. Dancer, Competition systems with strong interaction on a subdomain, Topol. Methods Nonlinear Anal. 37 (1) (2011) 37-53.

[5] E.N. Dancer, Boundary-value problems for weakly nonlinear ordinary differential equations, Bull. Aust. Math. Soc. 15 (1976) 321-328.

[6] E.N. Dancer, Y. Du, Competing species equations with diffusion, large interactions, and jumping nonlinearities, J. Differ. Equ. 114 (2) (1994) 434-475.

[7] D.G. de Figueiredo, J.-P. Gossez, On the first curve of the Fučík spectrum of an elliptic operator, Differ. Integral Equ. 7 (5-6) (1994) 1285-1302.

[8] P. Drábek, P. Nečesal, Nonlinear scalar model of a suspension bridge: existence of multiple periodic solutions, Nonlinearity 16 (3) (2003) 1165-1183.

[9] P. Drábek, S.B. Robinson, A new and extended variational characterization of the Fučík spectrum with application to nonresonance and resonance problems, Calc. Var. Partial Differ. Equ. 57 (2018) $1,27$.

[10] S. Fučík, Boundary value problems with jumping nonlinearities, Čas. Pěst. Mat. 101 (1) (1976) 69-87.

[11] S. Fučík, Solvability of Nonlinear Equations and Boundary Value Problems, Mathematics and Its Applications, vol. 4, D. Reidel Publishing Co., Dordrecht, 1980.

[12] F. Gazzola, Mathematical Models for Suspension Bridges. Nonlinear Structural Instability, vol. 15, Springer, Cham, 2015.

[13] G. Holubová, P. Nečesal, Nontrivial Fučík spectrum of one non-selfadjoint operator, Nonlinear Anal. 69 (9) (2008) 2930-2941.

[14] J. Horák, W. Reichel, Analytical and numerical results for the Fučík spectrum of the Laplacian, J. Comput. Appl. Math. 161 (2) (2003) 313-338. 
[15] J. Kadlec, P. Nečesal, The Fučík spectrum as two regular curves, in: Nonlinear Analysis and Boundary Value Problems, NABVP 2018, Santiago de Compostela, Spain, 4-7 September 2018, in: Proceedings of the International Conference. Dedicated to Juan J. Nieto on the Occasion of His 60th Birthday, Springer, Cham, 2019, pp. 177-198.

[16] P. Krejčí, On solvability of equations of the 4th order with jumping nonlinearities, Čas. Pěst. Mat. 108 (1) (1983) 29-39.

[17] A.C. Lazer, P.J. McKenna, Large scale oscillatory behaviour in loaded asymmetric systems, Ann. Inst. Henri Poincaré, Anal. Non Linéaire 4 (1987) 243-274.

[18] A.C. Lazer, P.J. McKenna, Large-amplitude periodic oscillations in suspension bridges: some new connections with nonlinear analysis, SIAM Rev. 32 (4) (1990) 537-578.

[19] J. Liesen, Z. Strakoš, Krylov Subspace Methods. Principles and Analysis, Reprint of the 2013 hardback edition, reprint of the 2013 hardback edition ed., Oxford University Press, Oxford, 2015.

[20] I. Looseová, P. Nečesal, The Fučík spectrum of the discrete Dirichlet operator, Linear Algebra Appl. 553 (2018) 58-103.

[21] R. Ma, Y. Xu, C. Gao, Spectrum of linear difference operators and the solvability of nonlinear discrete problems, Discrete Dyn. Nat. Soc. 2010 (2010) 27.

[22] C. Margulies, W. Margulies, Nonlinear resonance set for nonlinear matrix equations, Linear Algebra Appl. 293 (1-3) (1999) 187-197.

[23] C.A. Margulies, W. Margulies, An example of the Fučik spectrum, Nonlinear Anal., Theory Methods Appl. 29 (12) (1997) 1373-1378.

[24] R. Molle, D. Passaseo, New properties of the Fučík spectrum, C. R., Math., Acad. Sci. Paris 351 (17-18) (2013) 681-685.

[25] S.B. Robinson, Y. Yang, Discrete nonlinear equations and the Fučík spectrum, Linear Algebra Appl. 437 (3) (2012) 917-931.

[26] B.P. Rynne, The Fucik spectrum of general Sturm-Liouville problems, J. Differ. Equ. 161 (1) (2000) 87-109.

[27] N. Sergejeva, S. Pečiulytè, On Fučík type spectrum for problem with integral nonlocal boundary condition, Nonlinear Anal., Model. Control 24 (2) (2019) 261-278.

[28] P. Stehlík, Discrete Fuč́k spectrum - anchoring rather than pasting, Bound. Value Probl. 2013 (2013) 11. 